\title{
Photomediated Controlled Radical Polymerization
}

Xiangcheng Pan, ${ }^{1}$ Mehmet Atilla Tasdelen, ${ }^{2}$ Joachim Laun, ${ }^{3}$ Thomas Junkers, ${ }^{3,4, *}$ Yusuf Yagci, ${ }^{5,6, *}$ Krzysztof Matyjaszewski ${ }^{1, *}$

${ }^{1}$ Carnegie Mellon University, Department of Chemistry, Pittsburgh, Pennsylvania, 15213, United States

${ }^{2}$ Department of Polymer Engineering, Faculty of Engineering, Yalova University, 77100 Yalova, Turkey

${ }^{3}$ Polymer Reaction Design Group, Institute for Materials Research (IMO), Universiteit Hasselt, Martelarenlaan 42, 3500 Hasselt, Belgium

${ }^{4}$ IMEC division imomec, Wetenschapspark 1, 3590 Diepenbeek, Belgium

${ }^{5}$ Department of Chemistry, Istanbul Technical University, 34469 Maslak, Istanbul, Turkey

${ }^{6}$ Center of Excellence for Advanced Materials Research (CEAMR) and Department of Chemistry, Faculty of Science, King Abdulaziz University, 21589 Jeddah, Saudi Arabia

\begin{abstract}
Photomediated controlled radical polymerization is a versatile method to prepare, under mild conditions, various well-defined polymers with complex architecture, such block and graft copolymers, sequence-controlled polymers, or hybrid materials via surface-initiated polymerization. It also provides opportunity to manipulate the reaction through spatiotemporal control. This review presents a comprehensive account of the fundamentals and applications of various Photomediated CRP techniques, including atom transfer radical polymerization (ATRP), reversible addition-fragmentation chain transfer (RAFT), nitroxide mediated polymerization (NMP) and other procedures. In addition, mechanistic aspects of other photomediated methods are discussed.
\end{abstract}

\section{Keywords}

Photopolymerization; radical polymerization; ATRP; RAFT; NMP 


\section{Introduction}

1.1 Reversible-deactivation radical polymerization

1.2 Basic chemistry of photomediated processes

\section{Photomediated ATRP}

2.1 Cu-based systems in the presence of photoinitiators

2.2 Cu-mediated system in the absence of photoinitiators

2.3 Fe-mediated photoATRP

2.4 Ir-mediated photoATRP

2.5 Ru-mediated photoATRP

2.6 Au-mediated photoATRP

2.7 Metal-free ATRP

2.8 Applications

\section{Photomediated RAFT Polymerization}

3.1 Iniferter polymerization

3.2 RAFT initiated by conventional photoinitiators

3.3 Initiator-free photoRAFT

3.4 Photoinduced electron transfer (PET)-RAFT

3.5 Applications

\section{Photomediated NMP}

5. Other Controlled Radical Polymerizations

5.1 Photomediated CMRP

5.2 Photomediated ITP

5.3 Photomediated TERP

6. Radical intermediates in other photomediated controlled polymerizations

7. Combination of photomediated radical polymerizations and click processes

8. Surface-initiated photomediated radical polymerization

8.1 PhotoATRP

8.2 Photoiniferter and photoRAFT

8.3 Photomediated NMP

8.4 PhotoCRP in direct laser writing

\section{Summary and perspective}




\section{Nomenclature}

Đ: $\quad$ Dispersity

AA: $\quad$ Acrylic acid

AET: $\quad$ Associative electron transfer

AFM: $\quad$ Atomic-force microscopy

AIBN: $\quad 2,2^{\prime}$-Azobis(2-methylpropionitrile)

ARGET: Activators regenerated by electron transfer

ATRP: $\quad$ Atom transfer radical polymerization

BA: n-Butyl acrylate

BAPO: Phenylbis (2,4,6-trimethylbenzoyl)phosphine oxide

BDMAT: $\quad S, S^{\prime}$-bis $(\alpha, \alpha$ '-dimethyl- $\alpha$ ''-acetic acid $)$

BnBiB: $\quad$ Benzyl $\alpha$-bromoisobutyrate

BnMA: Benzyl methacrylate

BPN: 2-Bromopropionitrile

BPO: Benzoyl peroxide

Bpy: $\quad 2,2$ '-Bipyridine

Btp: 2-(2'-Benzothienyl)pyridine

BuMA: Butyl methacrylate

CEF: $\quad$ Chain end functionality

CMRP: Cobalt-mediated radical polymerization

CPEC: $\quad S$-2-Cyano-2-propyl- $O$-ethyl xanthate

CPFDB: 2-Cyanoprop-2-yl(4-fluoro)

dithiobenzoate

CRP: $\quad$ Controlled radical polymerization

CTA: Chain transfer agent

CuAAC: Copper(I)-catalyzed azide-alkyne cycloaddition

DCF: Dead chain fraction

DCPD: Dicyclopentadiene

DDMAT: $\quad S$-Dodecyl- $S$ '-( $\alpha, \alpha$ '-dimethyl- $\alpha$ '-acetic acid)

DET: $\quad$ Dissociative electron transfer

DLS: $\quad$ Dynamic light scattering

DLW: $\quad$ Direct laser writing

DMAEMA: 2-(Dimethylamino)ethyl methacrylate

DMF: $\quad$ Dimethylformamide

DMSO: Dimethyl sulfoxide

DP: $\quad$ Degrees of polymerization

Dppm: (Diphenylphosphino)methane

DT: Degenerative transfer

DVP: $\quad$ Dimethyl vinylphosphonate

EBiB: $\quad$ Ethyl $\alpha$-bromoisobutyrate

EBPA: Ethyl $\alpha$-bromophenylacetate
EDC: 1-Ethyl-3-(3-

dimethylaminopropyl)carbodiimide

EEMA: 2-Ethoxyethyl methacrylate

EGMA: Ethylene glycol methyl ether

methacrylate

EMA: $\quad$ Ethyl methacrylate

ESI: Electrospray-ionization

FTIR: $\quad$ Fourier transform infrared spectroscopy

GMA: Glycidyl methacrylate

HEMA: 2-Hydroxyethyl methacrylate

HMTETA: $1,1,4,7,10,10$ -

Hexamethyltriethylenetetramine

HPMA: $\quad N$-(2-Hydroxypropyl) methacrylamide

ICAR: Initiators for continuous activator regeneration

ITP: $\quad$ Iodine transfer polymerization

ISET: Inner-sphere electron transfer

LCST: Lower critical solution temperature

LFP: $\quad$ Laser flash photolysis

LRP: $\quad$ Living radical polymerization

MA: $\quad$ Methyl acrylate

MAA: Methacrylic acid

MBiB: Methyl 2-bromoisobutyrate

$\mathrm{Me}_{6}$ TREN: Tris[2-(dimethylamino)ethyl]amine

MESA: Methyl(ethoxycabonothioyl)sulfanyl acetate

MiB: $\quad$ Methyl isobutyrate

MIP: $\quad$ Molecularly imprinted polymer

MMA: Methyl methacrylate

MS: $\quad$ Mass spectrometry

$M_{n}: \quad$ number-average molecular weight

$M_{w:} \quad$ weight-average molecular weight

MW: $\quad$ molecular weight

MWD: molecular weight distribution

NAP: $\quad N$-(2-Acryloyloxyethyl)pyrrolidone

NIPAAm: $\quad N$-Isopropyl acrylamide

NMP: $\quad$ Nitroxide-mediated radical polymerization

NVP: $\quad N$-Vinyl carbazole

OEGA: Oligo(ethylene glycol) methyl ether acrylate

OEOMA: Oligo(ethylene oxide) methyl ether methacrylate 
OEGMA: Oligo(ethylene glycol) methyl ether methacrylate

OSET: $\quad$ Outer-sphere electron transfer

OMRP: Organometallic mediated radical polymerization

PEGA: Polyethylene glycol acrylate

PET: $\quad$ Photoinduced Electron Transfer

Ph: $\quad$ Phenyl

Phen: $\quad$ 1,10-Phenanthroline

PI: $\quad$ Photoinitiator

PISA: $\quad$ Polymerization-induced self-assembly

PLP: $\quad$ Pulsed-laser polymerization

PMA: $\quad$ Propargyl methacrylate

PMDETA: $N, N, N^{\prime}, N^{\prime \prime}, N^{\prime \prime}-$

Pentamethyldiethylenetriamine

PNB: $\quad$ Polynorbornene

Ppy: 2,2'-Phenylpyridine

PS: $\quad$ Photosensitizer

PTZ: $\quad$ 10-Phenylphenothiazine

QCM: Quartz crystal microbalance

RAFT: Reversible addition-fragmentation chain transfer

RDRP: Reversible-deactivation radical polymerization

ROMP: Ring-opening metathesis polymerization

RP: $\quad$ Radical polymerization

SARA: Supplemental activators and reducing agents

SAM: $\quad$ Self-assembled monolayer

SCE: $\quad$ Saturated calomel electrode
SDS: $\quad$ Sodium dodecyl sulfate

SIMS: $\quad$ Secondary ion mass spectrometry

SPMA: 3-Sulfopropyl methacrylate

SR\&NI: $\quad$ Simultaneous reverse and normal initiation

St: $\quad$ Styrene

STXM: $\quad$ Scanning transmission X-ray microscopy

TEM: Transmission electron microscopy

TEMPO: $\quad$ 2,2,6,6-Tetramethylpiperidinyloxy-4-yl radical

TERP: Telluride-Mediated Polymerization

TFEMA: 1,1,1-Trifluoroethyl methacrylate

Tmd: 2,2,6,6-Tetramethyl-3,5-heptanedione

TMEDA: Tetramethylethylenediamine

TMPTA: Trimethylolpropane triacrylate

TPO: $\quad$ 2,4,6-Trimethylbenzoyl)diphenyl phosphine

TPMA: $\quad$ Tris((2-pyridine)methyl)amine

TPMA*: $\quad$ Tris((4-methoxy-3,5-dimethylpyridin-2yl)methyl)amine

Tren: $\quad$ Tris(2-aminoethyl)amine

TTC: Trithiocarbonate

UV: Ultraviolet

VAc: Vinyl acetate

VBA: $\quad \alpha$-Vinylbenzyl alcohol

Vis: $\quad$ Visible

VL: $\quad$ Valerolactone

VP: $\quad$ Vinyl pivalate

XPS: $\quad$ X-ray photoelectron spectroscopy 


\section{Introduction}

\subsection{Reversible-deactivation radical polymerization}

Conventional radical polymerization is a widely used technique to synthesize polymeric materials, which is typically performed under various reaction conditions such as bulk, in organic solvents or water, under homogeneous and heterogeneous conditions at different temperatures.[1] However, conventional radical polymerization cannot be used to synthesize well-defined polymers with precise average molar masses, narrow molecular weight distributions and predesigned architectures because of unavoidable radical-radical terminations. Typical living polymerization techniques require a fast initiation and concurrent growth of all chains. To achieve living-like or controlled radical polymerization, several principles should be fulfilled: fast initiation, long lifetime of growing chains, and small fraction of terminated chains. A dynamic equilibrium between dormant species and growing radicals meets these criteria, and a process is termed as reversible deactivation radical polymerization (RDRP), or controlled radical polymerization (CRP).[2, 3] In "living" radical polymerization, there should always be some termination; however, a controlled radical polymerization can be accomplished by rapid exchange of growing radicals and dormant species, by increasing the concentration of dormant species or by reducing polymerization rate,. The dead chain fraction (DCF) is defined as the ratio of terminated chains (T) to all initiated chains (R-X), eq 1.[4] Decreasing DCF, i.e., increasing chain end functionality, could be realized by decreasing degree of polymerization $\left(\mathrm{DP}_{\mathrm{T}}\right)$, conversion $(p)$, or rate coefficients of termination to propagation $\left(k_{\mathrm{t}} / k_{\mathrm{p}}\right)$ and increasing initial monomer concentration $\left([\mathrm{M}]_{0}\right)$ and reaction time $(t)$.

$$
\mathrm{DCF}=\frac{[\mathrm{T}]}{[\mathrm{R}-\mathrm{X}]_{0}}=\frac{2 \mathrm{DP}_{\mathrm{T}} k_{\mathrm{t}}[\ln (1-p)]^{2}}{[\mathrm{M}]_{0} k_{\mathrm{p}}{ }^{2}}
$$

In general, there are three types of controlled radical polymerizations. The first type uses dormant species with a weak bond, which provides a propagating radical and a counter-radical $\left(\mathrm{T}^{*}\right)$ via a homolytic cleavage (Error! Reference source not found.a). The counter-radical is a rather stable radical. It can only trap a propagating radical and should not react with monomer. Commercially available nitroxyl radicals are used in a process, termed nitroxide mediated polymerization (NMP).[5] In a similar approach, organometallic mediated radical polymerization (OMRP) employs a weak bond between carbon and transition metal species, for example Coporphyrins, $[6,7]$ and derivatives of Fe or Mo.[8] 


\section{Insert Scheme 1.}

It is possible to convert the first type of CRP to a catalytic system by using alkyl halides and transition metal complexes, as in atom transfer radical polymerization (ATRP). [9-11] Transition metal complexes in lower oxidation state $\left(\mathrm{Mt}^{\mathrm{n}} / \mathrm{L}\right)$ activate functional alkyl bromide or chloride initiators to give initiating radical and deactivator complex in higher oxidation state $\left(\mathrm{X}-\mathrm{Mt}^{\mathrm{n}+1} / \mathrm{L}\right)$. The deactivator species deactivate propagating radicals to alkyl halides (dormant state) (Error! Reference source not found.b). Various metal complexes are used to catalyze ATRP including $\mathrm{Cu}, \mathrm{Fe}, \mathrm{Ru}, \mathrm{Ni}$, Os and other metals formed with amines, phosphines and other ligands, and copper catalyst with polydentate amine and pyridine based ligands is most used.[12]

The rate of ATRP is defined by eq 2, where radical concentration $\mathrm{P}_{\mathrm{n}}{ }^{*}$ is defined by the ATRP equilibrium constant $\left(K_{\mathrm{ATRP}}\right)$, concentration of alkyl halide (dormant species) and the ratio of activator and deactivator concentrations.[10] It is worth to point out that the rate does not depend on the absolute concentration of activator.

$$
R_{\mathrm{p}}=k_{\mathrm{p}}[\mathrm{M}]\left[\mathrm{P}_{n}^{*}\right]=k_{\mathrm{p}} K_{\mathrm{ATRP}}\left(\frac{\left[\mathrm{P}_{n} \mathrm{X}\right]\left[\mathrm{Cu}^{\mathrm{I}} / \mathrm{L}\right][\mathrm{M}]}{\left[\mathrm{X}-\mathrm{Cu}^{\mathrm{II}} / \mathrm{L}\right]}\right)
$$

According to above equation, the same ratio of activator to deactivator concentrations would result in the same overall rate, even with a very low concentration of catalyst. However, traditional ATRP used high concentration of the activator to maintain the acceptable polymerization rate because each radical termination leads to the irreversible loss of the activator due to the persistent radical effect. [12, 13] Several procedures with low catalyst loading below 100 or even below $10 \mathrm{ppm}$ were developed via regeneration of activator complex, as shown in Error! Reference source not found..[14] The reduction of deactivator in higher oxidation state was accomplished by addition of chemical reducing agents in activator (re)generation by electron transfer (ARGET) ATRP,[14] an external radical initiator in initiators for continuous activator (re)generation (ICAR) ATRP,[15] a current in electrochemically mediated eATRP,[16] zerovalent metals or sulfites in supplemental activators and reducing agents (SARA) ATRP,[1720] or photochemical reduction in photoATRP.[21-25] Recently, ATRP based on small organic molecule, called metal-free (MF), organocatalyzed $(\mathrm{O})$ or photoinduced electron transfer (PET) ATRP, was reported using alkyl bromide as ATRP initiator.[26, 27] A similar method using alkyl iodides with various organic catalysts was also reported to provide well-defined polymers.[28] 


\section{Insert Scheme 2.}

The third RDRP method is based on activation-deactivation process which utilizes a degenerative transfer (Error! Reference source not found.c) between transfer agents $\left(\mathrm{P}_{\mathrm{m}}-\mathrm{X}\right)$ and growing radicals $\left(\mathrm{P}_{\mathrm{n}}{ }^{*}\right)$. Similar to conventional radical polymerization, the radical is formed externally, and is not formed internally from the dormant specie. The externally supplied radical generates a small number of growing chains, and also the polymerization stops when the source of radicals is consumed. Various transfer agents were used in degenerative transfer systems, including alkyl iodides, derivatives of tellurium or bismuth,[29] and thiocarbonylthio compounds, such as dithioesters, trithiocarbonates, xanthates and dithiocarbamates.[30] The method with dithiocompound, called reversible addition-fragmentation chain transfer (RAFT) polymerization, undergoes an exchange via addition-fragmentation process (Error! Reference source not found.).[31] In RAFT, kinetics follows the steady state approach, similar to the behavior in a conventional radical polymerization, but linear evolution of molecular weight with conversion and narrow molecular weight distributions resemble a controlled process.

\section{Insert Scheme 3.}

Some experimentally quantifiable parameters for NMP, ATRP and RAFT were recently quantified and correlated.[32] Several borderline systems were also developed with both spontaneous and catalyzed cleavage, or degenerative transfer and ATRP or OMRP. It is interesting to note that equilibria between active in dormant species, typical for all RDRP systems, are also essential in many biological processes. [33]

\subsection{Basic chemistry of photomediated processes}

\subsubsection{Photoinitiators/photosensitizers}

Photoinitiated polymerization is a process that transforms monomers into polymers under ultra violet-visible (UV-Vis) light irradiation.[34] The polymerization is initiated by photogenerated reactive species, either free radicals or ions, which are produced from added photosensitive compounds, namely photoinitiators (PI) and/or photosensitizers (PS) (

Figure 1).

\section{Insert Figure 1.}


The key feature of photoinitiated polymerization is its ability to be conducted under mild reaction conditions. This provides several advantages, including more environmentally friendly and efficient processes and preparation of novel materials.[35, 36] Since the polymerization may proceed at room temperature, thermally sensitive molecules, such as pharmaceutical compounds, enzymes, peptides or proteins can be safely incorporated into the polymers during polymerization. Solvent-free formulation, or use of less solvent in the polymerization process, reduces the release of volatile compounds giving rise to environmental and health benefits. Unlike thermally initiated polymerization, photoinitiated polymerization provides spatial and temporal controls that make it possible to determine where, when and what type of polymerization process could be applied.[37] Moreover, the rate of initiation and polymerization can be controlled by adjusting light intensity or concentration of photosensitive compounds.

In the free radical mode, which is the focus of this article, photosensitive compounds generate active species either through unimolecular dissociation or bimolecular photoinduced electron transfer reactions upon exposure to UV or visible light.[38-40] A unimolecular photoinitiator undergoes a homolytic bond cleavage, producing active free radicals. In the case of a bimolecular photoinitiator, active free radicals are formed via a hydrogen abstraction or an electron-transfer mechanism between a photosensitizer, which absorbs the light and produces a long-lived excited triplet state, and a co-initiator that can quench the excited molecules (

Figure 2). There are many commercially available photoinitiators activated by UV and visible light irradiations and some of them are listed in Table 1.

\section{Insert Figure 2.}

\section{Insert Table 1.}

The main classes of unimolecular photoinitiators are benzoin ethers, benzil ketals, acetophenones, oxime esters, aminoalkyl phenones, and acylphosphine oxides. Typical bimolecular photoinitiators include benzophenones, thioxanthones, benzils, and quionones as photosensitizers

with alcohols, amines, and thiols acting as hydrogen donors. In addition to conventional free radical polymerization, photosensitive compounds have also been used for photochemical initiation of controlled radical polymerization procedures. In this context, photoinitiators, photosensitizers, photoredox catalysts play vital role in the process which will be detailed in the following sections. 


\subsubsection{Photoredox catalysts}

Photoinduced electron transfer from transition metals (e.g. ruthenium and iridium complexes) and organic dyes (e.g. eosin Y, 9,10-dicyanoanthracene, and triphenylpyrylium salts) have recently received considerable attention, particularly for synthetic applications in a process called photoredox organocatalysis,

Figure 3.[41] Due to their excellent light absorption properties (from $400 \mathrm{~nm}$ to $800 \mathrm{~nm}$ ), these catalysts could be applied in various specific organic reactions, namely the formation of carboncarbon bonds, including atom transfer radical addition to unactivated terminal alkenes, the reduction of organic halides, or the conversion of alcohols to the corresponding halides.[42]

\section{Insert Figure 3.}

These photoredox catalysts generate active species through single-electron transfer between excited state of a given metal complex or organic dye and an oxidative or reductive quencher under visible light irradiation. Upon light absorption, the photosensitizer (PS) attains a long-lived excited triplet state $\left(\mathrm{PS}^{*}\right)$ that can be either reductively quenched $\left(\mathrm{PS}^{\circ}{ }^{-}\right.$) or oxidatively quenched $\left(\mathrm{PS}^{\circ+}\right)$ by a wide range of organic substrates. The formed radical ions (substrate ${ }^{\circ+}$ ) are subsequently converted to the final products in a number of possible reactions (

Figure 4).[43]

\section{Insert Figure 4.}

Photoredox catalysts are activated under very mild conditions, with most reactions proceeding at room temperature without the need for reactive radical initiators. They exhibit wide functional group tolerance and minimization of waste products.[41] These catalysts were initially based on ruthenium or iridium compounds because of their intense visible light absorption, long lived excited states and suitable redox potentials. In general, they were utilized in (i) photodynamic therapy, (ii) dye-sensitized solar cells and (iii) organic light-emitting diodes, and (iv) polymerization reactions. Heterogeneous semiconductors such as mesoporous carbon nitride and various metal oxides and sulfides were also used as visible light photoredox catalysts.[44] 
However, their activation mechanisms were more complex then transition metal-polypyridyl complexes.

Very recently, several reviews on photo-initiated/mediated/induced polymerization have been published,[45-48] but this review highlights the recent progress in the use of light to regulate controlled/living radical polymerization from mechanism, reaction, material preparation to advanced applications.

\section{Photomediated ATRP}

\subsection{Cu-based systems in the presence of photoinitiators}

The significant progress made in controlled radical polymerization techniques during the past few years has enabled the synthesis of well-defined polymers by photochemical means. Preliminary examples of successful photomediated CRP were based on integration of photosensitive compounds (photoinitiators, photosensitizers or photoredox catalysts) in well-known CRP systems. The role of photosensitive compounds was to convert the physical energy of incident light into chemical energy in the form of reactive radicals with high electron-donating properties. These radicals were capable of regenerating a lower oxidation state activator from a higher oxidation state deactivator (

Figure 5).[49] Many UV and visible light sensitive compounds including unimolecular[50-53] and bimolecular free radical photoinitiators, [50, 54] dyes, [52] semiconducting nanoparticles[5560] and metal carbonyls[61] were used to photochemically initiate and control the ATRP process ( 
Table 2).

\section{Insert Figure 5.}

\section{Insert Table 2.}

Upon UV irradiation, unimolecular photoinitiators such as 2,2-dimethoxy-2-phenyl acetophenone or (2,4,6-trimethylbenzoyl) diphenylphosphine oxide undergo a unimolecular bond cleavage to generate free radicals, which could be used for the activation of an ATRP at room temperature. On the other hand, bimolecular photoinitiators such as benzophenone or camphorquinone, require a second compound called a co-initiator, including tertiary amines, thiols or alcohols, to produce such intermediates. Both reverse ATRP[62] and simultaneous reverse \& normal initiation (SR\&NI)[63] were investigated for photoinduced ATRP.[54] In the reverse ATRP system, the polymerization of methyl methacrylate (MMA) proceeded in the absence of an added alkyl halide, but with a poor control over molecular structure. The results also indicated that unimolecular photoinitiators were more suitable due to their mechanistic advantages including higher quantum yield and short lifetimes of transient steps, which prevented any quenching by the metal ions. Addition of alkyl halide to the mixture to provide a SR\&NI ATRP system, not only improved initiation efficiency but also produced well-defined polymers with more narrow molecular weight distribution (1.11 1.26).

Subsequently, a photoinduced ATRP was conducted in an inverse microemulsion media using hexane as the continuous phase and poly(oxyethylene) (3) oleyl ether and poly(oxyethylene) (6) oleyl ether surfactants along with aqueous oligo(ethylene glycol) monomethyl ether methacrylate solutions containing (poly(ethylene glycol) $\left(M_{\mathrm{n}}=550 \mathrm{~g} / \mathrm{mol}\right)$ as a costabilizer), tris((2pyridine)methyl)amine (TPMA) or $N, N, N^{\prime}, N^{\prime \prime}, N^{\prime \prime}$-pentamethyldiethylenetriamine (PMDETA) as ligand, with a water-soluble photoinitiator (Irgacure 2959) and an alkyl halide (poly(ethylene oxide) based macroinitiator) under UV irradiation.[53] Photochemically generated radicals from both photoinitiator and direct photolysis of the $\mathrm{Cu}(\mathrm{II}) /$ ligand complex not only initiated the polymerization but also regenerated the $\mathrm{Cu}(\mathrm{I})$ activators, which could be lost due to termination reactions. The method was particularly advantageous for tuning the final particle size by switching on/off the UV light and adjusting, the fraction of the aqueous phase in the recipe. The UV light enabled to control growth of polymer chains, while the polymer molecular weights were determined by the size of the micelles. 
Many attempts have been directed towards extending spectral sensitivity to longer wavelengths. The use of visible-light sensitive compounds such as photoinitiators, dyes, mesoporous carbon nitride and various metal oxides and sulfides were employed for the key step of regeneration of $\mathrm{Cu}(\mathrm{I})$ activator in an ATRP.[49] In one case, the excited dye molecule and hydrogen donor molecule such as amine underwent electron transfer reaction to form radical-cation/radical-anion pairs, which essentially yielded free radicals in the system.[52] The radicals produced from the hydrogen donors were able to activate the ATRP, but the other radicals generated from the dye molecules were not capable of reducing the $\mathrm{Cu}(\mathrm{II})$. They acted mostly as radical chain terminators or underwent other reactions leading to bleaching the dye. A unimolecular photoinitiator, (2,4,6-trimethylbenzoyl)diphenylphosphine oxide, could be also utilized for the visible-light induced ATRP and provided better control of the molecular characteristics under the same experimental conditions.[51] Considering their role in photoinduced click reactions and photodecomposition pathway, acylgermane photoinitiators were also expected to activate ATRP within the visible range.[64]

In another approach, the use of camphorquinone and benzhydrol as photosensitive compounds was investigated for visible light-induced ATRP.[54] In this system, the camphorquinone absorbed visible light and abstracted a hydrogen from benzhydrol to form two ketyl radicals, which were inactive toward the monomer, but were able to reduce $\mathrm{Cu}$ (II) to $\mathrm{Cu}$ (I) species. In addition, the PMDETA ligand also could act as hydrogen donor and produced free radicals that were active in the ATRP equilibrium. The reverse ATRP process was poorly controlled and only polymers with broad molecular weight distributions were obtained in the absence of benzhydrol. The addition of benzhydrol to the process provided better control over molecular characteristics, forming polymers with narrow molecular weight distributions and good agreement between theoretical and experimental molecular weights. The SR\&NI ATRP system was also investigated under similar conditions and was more successful than photoinduced reverse ATRP.

Zinc oxide[57], titanium dioxide[55, 60] and mesoporous carbon nitride[56] nanoparticles, are known light-sensitive compounds which absorb visible light and consequently released their electrons in the conductive band (

Figure 6). These photochemically generated electrons could be efficiently used to modulate an ATRP process by regenerating $\mathrm{Cu}(\mathrm{I})$ activators from $\mathrm{Cu}(\mathrm{II})$ deactivators. Recently, the 
photocatalytic activity of $\mathrm{TiO}_{2}$ or $\mathrm{ZnO}$ nanoparticles was improved by the addition of photosensitive compounds such as dyes, reduced graphene oxides and iron nanoparticles. The addition of these compounds not only extended the optical absorption characteristics of the system up to the visible range but also enhanced the rate of polymerization.[58] The process was also implemented for surface-initiated ATRP of various monomers, including sulfopropyl methacrylate potassium salt, 2-hydroxyethyl methacrylate, $N$-isopropylacrylamide and oligo(ethylene glycol) methacrylate in an aqueous environment. The visible light-initiated polymerization using $\mathrm{TiO}_{2}$ nanoparticles in the presence of an initiator-modified substrate resulted in polymer brush growth in a controlled manner.[55, 60] The thickness of the polymer brushes increased linearly as a function of irradiation time. An especially attractive feature of this system was the heterogeneous nature of the nanoparticles, which made them easily separable and removable from the reaction media without causing any contamination in the ATRP process. Furthermore, this system did not require any expensive compounds, metals, and conventional radical initiators that might lead to side reactions. In a recent study, the visible light-induced ATRP was successfully mediated by benzyl alcohol grafted niobium(V) chloride $\left(\mathrm{NbCl}_{5}\right)$ nanoparticles as a heterogeneous photocatalyst with ethyl 2-bromoisobutyrate as initiator led to the well-defined polymers with predicted molecular weights and narrow molecular weight distributions $\left(M_{\mathrm{w}} / M_{\mathrm{n}}=1.16 \sim 1.30\right)$. [59] The niobium complex nanoparticles could be reused as photocatalysts in five further cycles without significant loss of control or activity.

\section{Insert Figure 6.}

Dimanganese decacarbonyl $\left(\mathrm{Mn}_{2}(\mathrm{CO})_{10}\right)$ is a photosensitive compound that can homolytically decompose to manganese pentacarbonyl $\left({ }^{\circ} \mathrm{Mn}(\mathrm{CO})_{5}\right)$ when exposed visible light illumination or sunlight.[61] These radicals can not only abstract halogen atoms from alkyl halides to generate carbon centered radicals, but also regenerate the $\mathrm{Cu}(\mathrm{I})$ activators thereby allowing photoinduced ATRP of (meth)acrylates and styrene (

Figure 7). The polymerization was instantly stopped in the absence of light. Subsequent reexposure to visible light restored the growth of polymer chains with the same kinetic characteristics that were observed in the former light-on process. This versatile process was also applied to the synthesis of graft copolymers from commercially available poly(vinyl chloride) via the grafting from approach. The direct abstraction of halide atoms from poly(vinyl chloride) by 
$\left(\mathrm{Mn}(\mathrm{CO})_{5}\right)$ radicals enabled the grafting of MMA from the polymer backbone. Successful formation of a graft copolymer was confirmed by gel permeation chromatography with monomodal molecular weight distribution and a significant shift of the peak value toward higher molecular weight.

\section{Insert Figure 7.}

\subsection{Cu-mediated system in the absence of photoinitiators}

\subsubsection{Cu-mediated photoATRP}

Among the various polymerizations that can be grouped under the tern photoATRP, coppermediated polymerizations play one of the most important roles due to their close resemblance to the classical copper/amine ATRP reactions. In fact, the effect of light on classical ATRP was first mentioned in 2000.[65] A strongly enhanced rate of polymerization was then observed under the influence of light; however it took more than 10 years before this observation was further investigated. PhotoATRP via reduction of initially added copper(II) salt was reported in the presence of an excess of PMDETA.[21, 22] This methodology was further developed using PMDETA and TPMA derivatives, leading to a successful photoATRP with less than $100 \mathrm{ppm}$ copper catalyst to monomer.[23, 24] A photoinduced copper-mediated ATRP was later reported for polymerization of acrylates in the presence of an excess of tris[2-(dimethylamino)ethyl]amine $\left(\mathrm{Me}_{6} \mathrm{TREN}\right)($

Figure 8). [25, 66]

\section{Insert Figure 8.}

Copper-mediated photoATRP is a versatile technique that has been used for the polymerization of many substituted acrylates; including methyl, [23, 25, 56, 66-75] ethyl, [23, 25, 71] $n$-butyl, [25, 67, 69, 70] $t$-butyl,[25, 70] ethylene glycol methyl ether,[25, 67, 71, 72] oligo(ethylene glycol) methyl ether,[25, 67, 68] di(ethylene glycol) ethyl ether, hydroxyethyl,[69, 70] hydroxypropyl,[69] lauryl,[69] stearyl,[69] isooctyl,[69] solketal,[67, 69, 71] and glycidyl

acrylate.[69] Other monomers, such as $N$-isoproylacrylamide[60] [2(acryloyloxy)ethyl]trimethylammonium chloride,[76] 3-(dimethylamino)propyl[76] and 2- 
ethylhexyl[76] acrylate were successfully polymerized in surface initiated reactions (see section $8)$.

Polymerization of methacrylates is more challenging in copper-mediated photoATRP, since fewer monomers were reported. They include methyl methacrylate[21-25, 56, 58, 74, 75, 77-80], 2-methacryloyloxyethyl phosphorylcholine[81], glycidyl methacrylate[81], and oligo(ethyl glycol) methyl ether methacrylate.[23] The polymerization of 2-hydroxyethyl,[55] 3-sulfoproypl,[55] 2(dimethylamino)ethyl,[60] and tert-butyl methacrylate,[80] as well as [2(methacryloyloxy)ethyl]trimethylammonium chloride,[55] and $N$-(2hydroxyproyl)methacrylamide[82] were described but only for surface initiated reactions. Especially, a family of iminophenylboronate methacrylates was polymerized under photoATRP conditions at low temperature.[83] Styrene[25, 56] has also been polymerized via copperphotoATRP. Regarding the rate of polymerization, a correlation between light intensity, irradiation wavelength spectrum and copper/ligand concentrations exists for the respective monomers. The ligand and its specific molar ratio to $\mathrm{CuBr}_{2}$ plays an important role.

\subsubsection{Comparison of copper complexes}

The structure of the copper complex $\mathrm{Cu}^{\mathrm{II}}\left(\mathrm{L}_{\mathrm{n}}\right) \mathrm{X}$ has a significant impact on the polymerization due to the solubility of the formed complex $[21,67,68]$ and the potential participation of the ligand in the reduction process.[66, 84] An excess of amine ligand was often added to the reaction mixture.[22, 23, 25, 80] Nevertheless, copper catalyst concentrations in the range of and below 100 ppm were successfully used.[23-25] The lowest concentrations, below 1 ppm, were reported only for surface initiated ATRP (refer to section 8).[76, 82]

Most acrylate monomers were subjected to photoATRP using copper(II) bromide as the copper source and $\mathrm{Me}_{6}$ TREN as ligand in dimethyl sulfoxide (DMSO).[25, 67, 69, 71-74] Generally, very good control over molecular weight distribution was achieved for various monomers, in the presence of different solvents and light sources. In almost all reports, an excess of 6 to 1 was used for $\mathrm{Me}_{6}$ TREN to copper(II). Ratios of copper to Me ${ }_{6}$ TREN of 1:2 and 1:3 using ethyl $\alpha$ bromoisobutyrate $(\mathrm{EBiB})$ for the polymerization of methyl acrylate (MA) in DMSO provided good control over the reaction.[25] Equimolar ratios usually resulted in no polymer formation within 90 min reaction time. This observation was also confirmed by a similar reaction that no polymerization occurred during the first 4 hours of irradiation, but that subsequent irradiation led to a slow well-controlled polymerization,[74] with lower excess ligand.[25] 
Copper complex $\left[\mathrm{Cu}\left(\mathrm{Me}_{6} \mathrm{TREN}\right)\right]\left(\mathrm{O}_{2} \mathrm{CH}\right)\left(\mathrm{ClO}_{4}\right)$ was reported as an efficient catalyst for photoATRP.[73] With this copper(II)-formate complex, near-quantitative conversion of methyl acrylate within 2 hours was reached, while maintaining excellent end-group fidelity and low dispersities even with only $100 \mathrm{ppm}$ copper catalyst. Thereby, the long-term stability of the reported copper(II)-formate complex was underpinned by the possibility to chain extend the obtained polymers even after 6 months' storage of the complex solutions.

A $\left[\mathrm{Cu}(\text { phen })_{2}\right] \mathrm{Br}$ (phen: 1,10-phenanthroline) photocatalyst was employed for visible light mediated photoATRP.[78] Excellent control was achieved with only $80 \mathrm{ppm}$ catalyst, even if polymerization was slow due to the low light intensity of a $0.9 \mathrm{~W} \operatorname{LED}(\lambda=465 \mathrm{~nm})$ light source. $\left[\mathrm{Cu}(\text { phen })_{2}\right]^{+}$was not reactive enough to undergo thermal copper-mediated radical polymerization at room temperature, in contrast to a photoexcited form $\left[\mathrm{Cu}(\mathrm{phen})_{2}\right]^{+^{*}}$. The reaction was probably mediated by an oxidative quenching of the photoexcited copper catalyst and not by an aminebased reductive quenching. $[66,84]$ In the presence of a large excess of triethylamine, the reaction was significantly accelerated but was still much slower than a $\mathrm{CuBr}_{2} / \mathrm{Me}_{6}$ TREN based ATRP without excess ligand.[74]

In addition to copper(II) bromide based catalysts, copper(0), copper(I) bromide, and copper(II) chloride were used as the copper source.[25] Recently, the food supplement, copper(II) gluconate was used for photoATRP in the presence of additional $\mathrm{Me}_{6}$ TREN ligand.[72] This finding underlined the importance of the copper(II)-Me ${ }_{6}$ TREN complex as the deactivating mediator; incomplete substitution of gluconate with $\mathrm{Me}_{6}$ TREN resulted in a significant loss of control, or even an uncontrolled radical polymerization. A slow ligand exchange required to premix copper(II) gluconate and $\mathrm{Me}_{6}$ TREN prior to the reaction. This process could be accelerated when the mixture was heated or exposed to UV-light. A faster ligand exchange was observed when two equivalents of sodium bromide per equivalent of copper(II) were added, enabling direct photo polymerization without the requirement of premixing. Moreover, the presence of impurities in the food supplement did not impair the reaction speed nor the end-group fidelity or polymer dispersity.

Next to $\mathrm{Me}_{6}$ TREN, other amine ligands have also been investigated. Replacing $\mathrm{Me}_{6}$ TREN with tris(2-aminoethyl)amine (TREN) in a polymerization of methyl acrylate with EBiB as initiator and only 400 ppm copper(II) bromide in DMSO led to only a minor difference in control. However, the use of PMDETA resulted in a significant reduction in both polymerization rate and control.[25] Other aliphatic ligands such as TPMA (Error! Reference source not found.) and 
tris((4-methoxy-3,5-dimethylpyridin-2-yl)methyl)amine (TPMA*) were used in dimethylformamide (DMF).[23] While a significant reduction in polymerization rate was observed for both TPMA and TPMA* ligands, the reactions maintained low dispersity with only 100 ppm copper. A comparable reaction with $\mathrm{Me}_{6}$ TREN in DMSO was 10 times faster without compromising polymerization control.[25] In contrast to the success with aliphatic amines, the use of 2,2 '-bipyridine (bpy) as a ligand did not result in any polymerization.

\section{Insert Scheme 4.}

PhotoATRP of methacrylates was investigated with various copper complexes. The most common ligands employed were TPMA [23, 24, 74] and PMEDTA (Error! Reference source not found.). [21-24, 56, 75, 77, 79] $\mathrm{Me}_{6}$ TREN resulted in a loss of control and reduced polymerization rates.[25, 77] TPMA generally gave lower dispersities and faster polymerization rates compared to PMDETA, $[23,74]$ and the opposite effect was observed in anisole with copper concentrations above $100 \mathrm{ppm}$.[24] A reduction in polymerization rates and a loss of control was found for both 50 and $200 \mathrm{ppm} \mathrm{CuBr}_{2} / \mathrm{PMDETA}$ in anisole. Depending on the solvent and copper to ligand ratio a further reduction in copper concentration resulted in a loss of control and polymerization rate (

Figure 9).[24, 74] Interestingly, equimolar ratio of TPMA to $\mathrm{CuBr}_{2}$ gave very similar polymerization rates and molecular weight distributions.[74] Use of PMDETA as ligand works as well under equimolar conditions at $100 \mathrm{ppm} \mathrm{CuBr}_{2} \cdot[24,74]$ Additionally, $\mathrm{CuCl}_{2}$ was used with alkyl bromide initiators without compromising polymerization rate or control.[77] However, initiation with chlorinated initiators was generally too slow for photoATRP of methyl methacrylate.

\section{Insert Figure 9.}

In contrast to an ATRP with $\mathrm{CuBr}_{2}$ as the copper(II) source, the polymerization of methyl methacrylate in DMSO with $\mathrm{CuSO}_{4} \cdot 5 \mathrm{H}_{2} \mathrm{O}$ as the copper(II) source proceeded faster and provided better control when TPMA was used in excess.[77] The use of an organic copper(II) salt for the polymerization of methyl methacrylate with 4 equivalents TPMA per copper(II) was further investigated, since these catalysts feature higher solubility in organic solvents.[77] While there were no significant differences between $\mathrm{CuBr}_{2}$ and organic copper(II) salts detectable for conversions above $60 \%$, there were smaller differences for lower conversions, since the 
deactivator $\mathrm{CuBr}_{2}$ was generated in situ. The same behavior was also found for methyl acrylate.[66] The use of $\mathrm{CuBr}$ instead of $\mathrm{CuBr}_{2}$ resulted in almost identical results. [80] A very similar behavior was found when $\mathrm{CuO}$ was used instead of copper(II) salts. [74] When oxygen was present, the reaction started after an induction period which was strongly dependent on the amount of amine ligand. Subsequent to the induction period, the reaction followed first-order kinetics forming polymers with low dispersities.

\subsubsection{Influence of the light source in photoATRP}

Not only ligand and ligand concentrations, but also selection of light source has a very significant influence on photoATRP reactions. Since the absorption spectra of different copper complexes and reaction mixtures are generally different, the light source has to be selected accordingly.[23] In one of the earliest studies on photoATRP, various light sources were compared for the polymerization of methyl methacrylate using $100 \mathrm{ppm} \mathrm{CuBr}_{2}$ to monomer and TPMA* as ligand in DMF (

Figure 10).

\section{Insert Figure 10.}

Sunlight mediated and controlled the reaction most efficiently.[23] After 12 hours, the sunlightmediated reaction yielded $81 \%$ conversion with $M_{\mathrm{n}}=21,000$ and $Ð=1.09$. The same reaction mediated by LED $\left(\lambda=392 \mathrm{~nm} ; 0.90 \pm 0.05 \mathrm{~mW} / \mathrm{cm}^{2}\right)$ light required 27 hours to reach $71 \%$ conversion, and maintained slightly better control with $Ð=1.05$. In contrast, use of blue $(\lambda=450$ $\left.\mathrm{nm} ; 10.0 \pm 0.5 \mathrm{~mW} / \mathrm{cm}^{2}\right)$ and red $\left(\lambda=631 \mathrm{~nm} ; 8.9 \pm 0.5 \mathrm{~mW} / \mathrm{cm}^{2}\right)$ light resulted in only $26 \%$ and $0 \%$ conversion, respectively, after more than 20 hours, as these wavelengths lay mostly outside the absorption spectrum of the copper complex.

The use of light at various wavelengths was also investigated for the polymerization of methyl acrylate using $\mathrm{Me}_{6}$ TREN as ligand, $\mathrm{EBiB}$ as initiator, and copper(II) bromide as catalyst precursor at various concentrations (down to $100 \mathrm{ppm}$ ) in DMSO.[25] The photoATRP with UV light (360 nm; $4 \times 9 \mathrm{~W}$ nail curing device) gave a significantly faster polymerization and access to a broader range of copper to initiator ratios than sunlight. In agreement with previous studies,[23] blue light resulted in a good polymerization control with slower polymerization rates.[25] 
Generally, higher light intensities provide faster chain activation, and faster polymerization. However, high intensities also resulted in side reactions, providing poorer control. Important to note is that following Lambert-Beer law strong light intensity gradients exist in reaction vessels, leading to almost $100 \%$ depletion of UV light within a distance of few millimeters. Only by intensive stirring, all material can be brought into the illumination area of the vessel, and hence polymerization rate and the level of control also depends on the geometry of the reactor and the positioning of the lamps.[74]

Temporal control is one of the biggest advantages of photoreactions over thermal procedures. In principle, no reaction should occur in the absence of light and reactions should immediately stop when light irradiation is switched off. Temporal control is usually assessed by sequential light-on light-off experiments. Indeed, in most studies, a strongly reduced polymerization rate but not a full cessation was observed during the light-off periods (

Figure 11).[21, 24, 25, 56, 61, 74, 79] Recently, a limited temporal control was observed during light-off periods.[73] Incomplete cessation of polymerization in dark time periods hints at formation of active $\mathrm{Cu}(\mathrm{I})$ species that persist and which then further activate dormant species.

\section{Insert Figure 11.}

\subsubsection{Initiators in photoATRP}

In absence of an ATRP initiator, only low conversions and/or uncontrolled polymerizations were observed for photoATRP reactions.[22-25, 56, 66, 74, 79, 80] Higher initiator concentration lead to faster polymerizations.[66] EBiB was used in basically all photoATRP of acrylates, but also 2,3-dihydroxypropyl 2-bromo-2-methylpropionate and 2-(2-hydroxyethoxy)ethyl 2-bromo-2methylpropinate initiated polymerizations successfully. Bifunctional initiators, such as ethylene bis(2-bromoisobutyrate and bis[2-(2'-bromoisobutyryloxy)ethyl]disulfide, were successfully used for the synthesis $\alpha, \omega$-telechelic block copolymers. However, increasing the number of initiation sites per molecule led to a higher degree of termination compared to monofunctional initiators.[67]

In contrast to the polymerization of acrylates, a variety of initiators were successfully employed for polymerization of methacrylates. Ethyl $\alpha$-bromophenylacetate (EBPA) and 2bromopropanitrile (BPN) initiated the polymerization efficiently and yielded polymers with lower dispersities compared to $\mathrm{EBiB}$ for the polymerization of methyl methacrylate.[23, 24] In 
accordance to the above described influence of $\mathrm{CuCl}_{2}$ on photoATRP, chlorinated initiators led to slower polymerization and poorer control due to slow initiation. [77, 85]

\subsubsection{Solvents in photoATRP}

A sufficient solubility of the copper complex species in the polymerization medium is very important for an effective reaction to proceed, and also solvent polarity plays an important role in the success of polymerizations. For example, bulk methyl methacrylate polymerization did not show good control due to insufficient solubility of the copper complex.[69, 77] When methanol was added to the polymerization mixture, much better results were obtained.[21] Most photoATRP reactions of acrylates were carried out in DMSO (25-50 vol\%),[25, 56, 66, 67, 69-75, 86] as well as in ionic liquids,[68] acetonitrile, [25, 56] DMF,[23, 25, 69] and other solvents such as toluene and alcohols have been reported.[25, 69] A variety of solvents were screened under similar conditions, and methanol was found to yield best results with near-quantitative conversion of monomer with $Ð=1.05$ within $90 \mathrm{~min}$,[25] whereas the use of other alcohols, i.e. ethanol and 2,2,2-trifluoroethanol only resulted in 38\% and 54\% conversion, respectively.[69] In contrast, the use of toluene as solvent resulted in a relatively uncontrolled polymerization with $Ð=1.54$ at $62 \%$ conversion after $90 \mathrm{~min} .[25]$

When water was used as solvent for the polymerization of oligo(ethylene glycol) methyl ether acrylate $\left(\mathrm{OEGA}_{480}\right)$ poor control over the polymerization was observed,[69] while the same reaction proceeded well in DMSO. Nevertheless, a 1 to 1 mixture of water and DMSO provided polymerization rates similar to pure DMSO and a further increase towards pure DMSO improved the control. Various ionic liquids were also identified as excellent solvents for polymerization of methyl acrylate, enhancing the polymerization rate by factor 3 and concomitantly giving better control for lowest copper catalyst loadings.[68] Other monomers, or resulting polymers, showed poor solubility in at least some of these ionic liquids, limiting their utility for block copolymerizations. The use of 1-octyl-3-methylimidazolium hexafluorophosphate $\left[\mathrm{C}_{8} \mathrm{mim}\right]\left[\mathrm{PF}_{6}\right]$ was beneficial for many monomers and allowed block copolymerization without compromising fast polymerization rates or dispersity.

Methyl methacrylate was predominantly polymerized in DMF,[23, 58, 75, 77, 79] and rarely in DMSO,[74, 77] anisole,[24, 74, 77] acetonitrile,[56] methanol,[21, 81], 1-butyl-3-methylimidazolium hexafluorophosphate $[\mathrm{bmim}]\left[\mathrm{PF}_{6}\right],[79]$ or in the absence of solvent. [22, 56] PhotoATRP of MMA performed with BPN as initiator and $100 \mathrm{ppm} \mathrm{Cu}\left[\mathrm{TPMA}_{\mathrm{Br}} \mathrm{Br}_{2}\right.$ in DMSO was significantly faster than when the polymerization was performed in anisole under same 
conditions.[24, 74] Recently, photoATRP of oligo(ethylene oxide) methyl ether methacrylate (OEOMA) using $\mathrm{CuBr}_{2}$ /TPMA as catalyst in aqueous media was reported.[87] In this study, TPMA was selected as ligand due to formation of a stable $\mathrm{Cu}(\mathrm{I})$ complex that does not undergo significant disproportionation.[88] The polymerization was originally investigated using poly(ethylene oxide) bromophenylacetate $\left(\mathrm{PEO}_{2000} \mathrm{BPA}\right)$ as macroinitiator with a high concentration of $\mathrm{Cu}(20,000 \mathrm{ppm}$ vs. monomer, $\mathrm{Cu}$ to initiator: 9:1) in very highly diluted solution (90\% water). The conversion of $\mathrm{OEOMA}_{500}$ reached $60 \%$ after $4 \mathrm{~h}$ of irradiation with visible light, providing polyOEOMA 500 with $M_{\mathrm{n}}=97,000, M_{\mathrm{w}} / M_{\mathrm{n}}=1.07$. Good control was maintained with $\mathrm{Cu}$ concentration as low as 220 ppm; however, the reaction with 110 or 22 ppm resulted in an uncontrolled radical polymerization. This was attributed to the dissociation of the halide from $\mathrm{CuBr}_{2}$ /TPMA complex, resulting in a low concentration of active deactivator, a major challenge for ATRP in aqueous media. $[89,90]$ This problem was resolved by adding $5 \mathrm{mM}$ or 30 $\mathrm{mM} \mathrm{NaBr}$ into the reaction mixture. The photoinduced ATRP of OEOMA $\mathrm{A}_{500}$ with $22 \mathrm{ppm} \mathrm{Cu}$ catalyst and $30 \mathrm{mM}$ salt after $4 \mathrm{~h}$ gave $55 \%$ conversion, and formed a polymer with $M_{\mathrm{n}}=103,000$ and $M_{\mathrm{w}} / M_{\mathrm{n}}=1.17$.

\section{Insert Figure 12.}

This system featured low concentrations of catalyst, as low as $c a .20 \mathrm{ppm}$ molar ratio to monomer, or $0.3 \mathrm{mg} / \mathrm{L}$ copper amount in aqueous media with irradiation by visible light at room temperature, which could be an attractive procedure for preparation of water soluble copolymers for a broad range of applications. A similar system using $\mathrm{Me}_{6}$ TREN as a ligand in aqueous media was recently reported to polymerize acrylates. [91]

\subsubsection{Mechanism of Cu-mediated photoATRP}

Several investigations were reported to elucidate the mechanism of photoATRP.[23, 25, 66, 84] In the early work, the activation rate of alkyl halides by $\mathrm{Cu}^{\mathrm{I}} / \mathrm{HMTETA}$ or TPMA catalyst was measured, and negligible difference was found in activation rate with or without irradiation. [23, 92] In contrast to the $\left[\mathrm{Cu}(\text { phen) })_{2}\right] \mathrm{Br}$ system (see section 2.2.1),[78] $\mathrm{CuBr}_{2}$ with TPMA or $\mathrm{Me}_{6}$ TREN was proposed to undergo an reductive quenching cycle, Error! Reference source not found.. No polymerization of MA was observed in the absence of free ligand or an appropriate electron donor such as trialkylamine, i.e., at $\mathrm{CuBr}_{2}: \mathrm{Me}_{6} \mathrm{TREN}=1: 1$, whereas increasing the ratio of ligand to copper resulted in an increased rate of polymerization, 
Figure 13.[66]

\section{Insert Scheme 5.}

\section{Insert Figure 13.}

A series of experiments and kinetic simulations were conducted in which one or more components were removed from the standard model conditions. [66] It was determined that the major pathway to radical (re)generation was the photochemical reduction of $\mathrm{Cu}^{\text {II }}$ complexes by free amine moieties, similar to an ARGET ATRP process. Small contributions from other processes such as the interaction between alkyl halides and ligands, ligands with monomers, and the photochemical cleavage of alkyl halides were still present. These observations were further supported by a more detailed investigation at a molecular level.[84] Pulsed-laser polymerization (PLP) was used to carry out the polymerization, and electrospray-ionization mass spectrometry (ESI-MS) was utilized to analyze the exact structure of the polymer and its chain end groups. In analogy to a previous report,[66] different combinations of photoATRP reaction components $\mathrm{EBiB}, \mathrm{CuBr}_{2}, \mathrm{Me}_{6} \mathrm{TREN}, \mathrm{MMA}$ and DMSO were investigated and led to a similar conclusion.[84]

PhotoATRP was also conducted with two fluorescent lamps $(\lambda=400-750 \mathrm{~nm})$ at a distance $10 \mathrm{~cm}$ from the reaction mixture.[80] This study raised one important question, whether photochemical processes influence various systems such as normal, ICAR and ARGET ATRP. ICAR ATRP polymerizations were conducted under ambient lighting or in the dark, and negligible differences were observed.[93] This suggested that under typical ICAR conditions, only chemical decomposition of AIBN contributes to the activator (re)generation. PhICAR (photochemical plus ICAR) ATRP was then investigated to clarify the effect of light on the activator (re)generation procedure. Previous photo-system used a light source at a distance of $10 \mathrm{~cm}$, while the following reactions conducted the reaction at $1 \mathrm{~m}$ distance from the light source. At low concentration of 2,2'-azobis(2-methylpropionitrile), AIBN, 0.035 equiv. with respect to ATRP initiator, photochemical process in $392 \mathrm{~nm}$ photoreactor $\left(0.9 \mathrm{~mW} / \mathrm{cm}^{2}\right)$ contributed $c a .50 \%$ to activator regeneration while at higher concentration of AIBN, 0.2 equiv with respect to ATRP initiator, the contribution dropped to $c a$. $15 \%$.

The effects of ambient light on normal and ARGET ATRP were also investigated.[94] An enhancement in polymerization rate was observed with ambient light under normal ATRP conditions, but not under ARGET ATRP conditions. Under both conditions, excess amine ligand 
PMDETA was used. The rate enhancement in normal ATRP could be due to (re)generation of activator $\mathrm{Cu}(\mathrm{I})$ via photoreduction of $\mathrm{Cu}$ (II) by excess ligands. In ARGET ATRP, the reduction of $\mathrm{Cu}$ (II) by chemical reducing agents such as ascorbic acid might be faster than photoreduction with excess amines. Therefore, the contribution of photochemical process is negligible, and no rate enhancement was observed in ARGET ATRP.

\subsection{Fe-mediated photoATRP}

PhotoATRP systems based on iron catalysts were reported in 2014 [95, 96] and 2015.[97-100] Iron could be considered as a preferred transition-metal for ATRP due to its abundance, lower toxicity, and lower cost than copper.[101, 102] Iron-mediated ATRP successfully polymerized various methacrylates and styrenes; however, polymerizations of acrylates were more challenging.[103-108] The Fe-mediated photoATRP polymerization of various methacrylates including methyl[97-100], ethyl[98, 100], n-butyl[98, 100], t-butyl[98], isobutyl[98], glycidyl[98], benzyl[100], 2-(trimethylsilyloxy)ethyl[100], and furfuryl methacrylate[100] were reported.

\section{Insert Table 3.}

PhICAR ATRP of MMA was conducted using $\mathrm{CCl}_{4}$ as ATRP initiator, tetramethylethylenediamine (TMEDA) as ligand and $\mathrm{FeCl}_{3}$ and $\mathrm{AIBN}$ as photoinitiators.[95] Benzoyl peroxide (BPO) was also successfully used as a conventional radical initiator under irradiation conditions. Typical features of controlled radical polymerization were observed in this system, such as linear semilogarithmic plots, linear relationships of $M_{\mathrm{n}}$ with conversion and low dispersity. Additionally, it was observed that $\mathrm{FeCl}_{3} / \mathrm{bpy}$ complex was reduced by ethanol under irradiation at room temperature.[95] A series of Fe-mediated photoATRPs were conducted by using this photoreduction mechanism to provide well-defined PMMA. An induction period was usually observed at the early stage of the polymerization period, and polymerization completed stopped in the absence of irradiation.

\section{Insert Figure 14.}

Nanosized $\alpha-\mathrm{Fe}_{2} \mathrm{O}_{3}$ was used as photoinitiator for the photoATRP of MMA with $\mathrm{FeCl}_{3} / \mathrm{PPh}_{3}$ as catalyst in PEG as solvent.[97] The polymerization proceeded in a controlled manner; however the dispersities of the synthesized PMMAs ranged from 1.39 to 1.68. It was proposed that under irradiation, an electron $\left(\mathrm{e}_{\mathrm{CB}}{ }^{-}\right)$and a positive hole $\left(\mathrm{h}_{\mathrm{VB}}{ }^{+}\right)$were generated in the $\alpha-\mathrm{Fe}_{2} \mathrm{O}_{3}$ nano- 
particle, and $\mathrm{Fe}(\mathrm{III})$ was reduced to $\mathrm{Fe}(\mathrm{II})$ by the electron $\left(\mathrm{e}_{\mathrm{CB}}^{-}\right)$. Once $\mathrm{Fe}(\mathrm{II})$ was formed, an ATRP equilibrium could be established.

Photoredox catalysts were extensively used in organic and material chemistry.[41, 109-113] An iron based catalyst, $\mathrm{Fe}(\mathrm{bpy})_{3} \mathrm{Br}_{2}$, was used to activate alkyl bromide to generate the radical in enantioselective organocatalytic alkylation of aldehydes.[114] A similar iron-based photoredox catalyst $\mathrm{Fe}(\mathrm{bpy})_{3}\left(\mathrm{PF}_{6}\right)_{2}$ was investigated for photoATRP of various methacrylates, [98] providing both linear semilogarithmic plot and a linear relationship of $M_{\mathrm{n}}$ with conversion. However, the molecular weights of the polymers obtained were ca. 10 times higher than theoretic values, indicating limited initiation efficiency.

Three iron-based photoredox catalysts were designed, synthesized with reduction potentials from -2.1 to $-2.7 \mathrm{~V}$ for the excited states of the catalysts.[99] With these reduction potentials, alkyl bromides such as EBPA could be reduced to radicals, initiating an ATRP. The "living" nature of this system was confirmed by linear kinetic plot, linear correlation between $M_{\mathrm{n}}$ and conversion, and clean chain extension. The broad molecular weight distribution $\left(M_{\mathrm{w}} / M_{\mathrm{n}}=1.5-2.2\right)$ for this system could be due to photo-degradation of iron photoredox complexes during polymerizations.

Polar solvents such as DMF and MeCN are efficient ligands for $\mathrm{FeBr}_{2}$-catalyzed ATRP of MMA.[108] Based on this observation, a photoinduced Fe-mediated ATRP of methacrylates using only air-stable $\mathrm{FeBr}_{3}$ without additional ligands, reducing agents or radical initiators was reported.[100] Simple irradiation of a reaction mixture of methacrylate, $\mathrm{FeBr}_{3}$ and EBPA in $\mathrm{MeCN}$ provided well-defined polymers with high bromine chain-end functionality. A mechanistic investigation showed that irradiation resulted not only in the photoreduction of $\mathrm{Fe}$ (III) to $\mathrm{Fe}$ (II) in presence of excess monomer (

Figure 15) but also in photoactivation of Fe(II) activators. Since the activation required irradiation, full temporal control with no detectable monomer conversion was observed when the irradiation was turned off.

\section{Insert Figure 15.}

\subsection{Ir-mediated photoATRP}

Inspired by powerful organic transformations reported by MacMillan,[41, 115] Yoon,[42, 116] and Stephenson,[117, 118] ATRP was successfully photocatalyzed using the most commonly 
used photoredox catalyst, fac-[Ir(ppy) $\left.{ }_{3}\right]$ (ppy: 2,2'-phenylpyridine).[119] Initially, the polymerization of MMA was investigated using EBPA as conventional ATRP initiator and 0.2 mol\% $\mathrm{fac}_{-}\left[\operatorname{Ir}(\mathrm{ppy})_{3}\right]$ as catalyst under visible light irradiation which resulted in an uncontrolled polymerization. This indicated that the photoredox catalyst was only involved in activating dormant species rather than deactivating the propagating radicals. This was also previously observed in photoinduced uncontrolled radical and ring-opening polymerizations using Ir photoredox complexes.[120] Interestingly, decreasing the loading of the Ir complex catalyst to $0.005 \mathrm{~mol} \%$ resulted in excellent control over the polymerization, providing PMMA with $M_{\mathrm{n}}=$ 22,900 and $M_{\mathrm{w}} / M_{\mathrm{n}}=1.25$. Another interesting property of this system was polymerization of methacrylic acid (MAA), which is one of the most challenging monomers to be polymerized under ATRP conditions due to a carboxylic acid functional group. Other methacrylates such as ethylene glycol methyl ether (PEGMA),[121] 1,1,1-trifluoroethyl (TFEMA),[122] and propargyl methacrylate (PMA)[123] were also polymerized in a controlled fashion under Ir-catalyzed photoATRP conditions.

\section{Insert Figure 16.}

Polymerization proceeded only under irradiation and in the presence of $f a c$ - $\left[\operatorname{Ir}(\mathrm{ppy})_{3}\right]$, and the reaction completely stopped by turning off the light, indicating that the excited state of Ir catalyst activated the alkyl bromide.[119] The simplified proposed mechanism for photoredox catalyst Ircatalyzed ATRP is shown in Error! Reference source not found.. A very strong reductant fac$\left[\operatorname{Ir}(\mathrm{ppy})_{3}\right]^{*}\left(E^{\Theta}=-1.73 \mathrm{~V}\right.$ vs SCE$)$ is formed under irradiation by visible light. This excited $\operatorname{Ir}^{\mathrm{III} *}$ species would reduce an alkyl bromide or polymer with bromine as chain end functionality to generate the propagating radical. The radicals then added monomers before being deactivated by the oxidizing $\mathrm{Ir}^{\mathrm{IV}}$ complex. The deactivation reaction between $\mathrm{Ir}^{\mathrm{IV}}$ complex and radicals closed the catalytic cycle to regenerate the ground-state Ir $^{\mathrm{III}}$ catalyst and dormant polymer chain with bromine chain end, which was important for a controlled radical polymerization.

\section{Insert Scheme 6.}

\section{Insert Table 4.}

Ir-catalyzed photoATRP was then extended to the polymerization of various acrylates including methyl, ethyl, n-butyl, t-butyl acrylates as well as acrylic acid in the presence of benzyl $\alpha$ bromoisobutyrate (BnBiB).[124] Random copolymerization of acrylic acid (AA) and ethyl 
acrylate (EA) was prepared at different molar ratios, up to $50 \mathrm{~mol} \%$ AA. In the polymerization with low ratios of AA (5 - 20 mol\%), copolymers with low dispersities, between 1.25 and 1.40 were achieved but higher dispersities $(1.51-1.90)$ were observed with higher ratios of AA (30 $50 \mathrm{~mol} \%$ ). Compared to the polymerization of MAA under Ir-catalyzed photoATRP conditions, control over the copolymerization of AA was more difficult to achieve. This could be related to the displacement of the terminal halide by a pendant nucleophilic carboxylate.[125]

A new iridium complex $\operatorname{Ir}(\mathrm{btp})_{2}(\mathrm{tmd})$ (btp: 2-(2'-benzothienyl)pyridine; tmd: 2,2,6,6-tetramethyl3,5-heptanedione) was designed and investigated for radical or cationic controlled polymerizations under mild irradiation $(\lambda$ : 457 - $532 \mathrm{~nm})$.[126] Compared to $f a c$-[ $\left.\operatorname{Ir}(\mathrm{ppy})_{3}\right]$, $\operatorname{Ir}(\mathrm{btp})_{2}(\mathrm{tmd})$ catalyst complex exhibited a stronger absorption in visible light range, and the luminescence lifetimes of newly designed complex $\operatorname{Ir}(\mathrm{btp})_{2}(\mathrm{tmd})$ was longer than $f a c$ - $\left[\operatorname{Ir}(\mathrm{ppy})_{3}\right]$ (4400 ns vs. 1300 ns). Consistent with previous observations, the photoATRP with higher loading of Ir catalyst, $1.5 \%$ mol ratio to initiator, resulted in an uncontrolled radical polymerization of MMA, but simply reducing the amount of catalyst to $0.14 \mathrm{~mol} \%$ ratio to initiator resulted in much better controlled polymerization, providing linear relationship between $M_{\mathrm{n}}$ and conversion as well as polymers with low dispersities $(1.2-1.3)$.

\subsection{Ru-mediated photoATRP}

Another photoredox catalyst tris(bipyridine)ruthenium(II) chloride $\left(\mathrm{Ru}(\mathrm{bpy})_{3} \mathrm{Cl}_{2}\right.$,

Figure 17) was recognized as a photosensitizer capable of initiating a free radical polymerization in the presence of a sacrificial electron donor trimethylamine in 1985.[127] A combination of $\mathrm{Ru}(\mathrm{bpy})_{3} \mathrm{Cl}_{2}$ and $\mathrm{N}, \mathrm{N}$-diisopropylethylamine was used to initiate radical polymerization in the presence of $\mathrm{EBiB}$ under visible light irradiation in 2011.[128] This system efficiently polymerized various methacrylates, including MMA, EMA, nBMA, tBMA, iBMA, GMA, and 2ethoxyethyl (EEMA) methacrylate. However, polymerization of styrene (St) and acrylates using this system was less successful.

\section{Insert Figure 17.}

At the same time, a cyclometalated ruthenium (II) complex was reported to catalyze photoATRP utilizing a different photo-activation mechanism.[129] A series of cyclometalated ruthenium (II) compounds were synthesized, and both $c i s-\left[\mathrm{Ru}\left(o-\mathrm{C}_{6} \mathrm{H}_{4}-2-\mathrm{py}\right)(\mathrm{phen})(\mathrm{MeCN})_{2}\right] \mathrm{PF}_{6}(\mathbf{R u I}$, 
Figure 17) and cis-[Ru(o- $\left.\left.\mathrm{C}_{6} \mathrm{H}_{4}-2-\mathrm{py}\right)(\mathrm{bpy})-(\mathrm{MeCN})_{2}\right] \mathrm{PF}_{6}(\mathbf{R u I I}$,

Figure 17) were photosensitive.[130] Both compounds were inactive under thermal conditions. However, visible light irradiation of complex $\mathbf{I}$ in methanol resulted in the dissociation of one acetonitrile ligand generating a reactive 16-electron $\mathrm{Ru}$ intermediate I'. The Ru complex I' with one vacant site activated an alkyl halide to produce a carbon-centered radical and a 17 electron complex II, which acted as an efficient deactivator in a classic ATRP equilibrium (Error! Reference source not found.), allowing polymerization of typical monomers, such as MMA, BA and St, with good control. Almost linear semilogarithmic kinetic plots for each monomer indicated a constant concentration of radicals during the polymerization under irradiation. Although the initiation efficiency was not quantitative, the $M_{\mathrm{n}}$ increased linearly with the monomer conversion. PMMA with a low value for $M_{\mathrm{w}} / M_{\mathrm{n}}$ (1.22) was synthesized, but polymerization of $n$-butyl acrylate (BA) and St resulted in formation of polymers with higher dispersity, $M_{\mathrm{w}} / M_{\mathrm{n}} 1.5-1.6$.

\section{Insert Scheme 7.}

\subsection{Au-mediated photoATRP}

Recently, a photoATRP system based on a dinuclear gold (I) photoredox catalyst $\left[\mathrm{Au}_{2}(\mu-\right.$ $\mathrm{dppm})_{2} \mathrm{Cl}_{2} \quad$ (dppm: (diphenylphosphino)methane) was reported.[131] Controlled radical polymerizations of methacrylates and acrylates were achieved in the presence of EBPA as the conventional ATRP initiator and low loadings, $1.25 \mathrm{~mol} \%$ ratio of $\mathrm{Au}(\mathrm{I})$ catalyst to initiator, under various irradiation conditions. Similar to the Ir-mediated photoATRP (Error! Reference source not found.), an excited state $\mathrm{Au}_{2}{ }^{\mathrm{I} *}$ complex ( $E^{\ominus}=-1.6 \mathrm{~V}$ vs SCE) was photogenerated, which reduced a $\mathrm{C}-\mathrm{Br}$ bond at the polymer chain end or in a low molecular weight initiator to provide a carbon-centered radical. This activation process was further supported by a rate constant of $9.2 \times 10^{8} \mathrm{M}^{-1} \mathrm{~s}^{-1}$ for the reaction between $\mathrm{Au}_{2}{ }^{{ }^{I}{ }^{*}}$ and EBPA by laser flash photolysis (LFP) measurements. Additionally, the oxidized gold complex $\left[\mathrm{Au}_{2}(\mu-\mathrm{dppm})_{2} \mathrm{Cl}_{2}\right]^{+} \mathrm{Br}^{-}$, detected by UV-vis spectra, quenched the propagating radical to regenerate the bromine chain end and ground-stated $\mathrm{Au}_{2}{ }^{\mathrm{II}}$ complex. The "living" nature of this process was confirmed by the 
predictable $M_{\mathrm{n}}$ and retention of chain end functionality (CEF). However, the molecular weight distributions were broad (1.62 - 1.85). This indicated that the deactivation was not very efficient and some side reactions competed with the main reaction pathways.

\subsection{Metal-free ATRP}

\subsubsection{Polymerization of methacrylates}

Inspired by the success of transition metal-based photoredox catalysts, a series of organic based small molecules were examined as catalysts for photoATRP, due to their intrinsic photoredox properties. 10-Phenylphenothiazine (PTZ,

Figure 18) was reported to successfully polymerize methacrylates in a controlled manner with traditional ATRP initiator using $380 \mathrm{~nm}$ irradiation.[26] When the reaction was conducted in the absence of irradiation, initiator or catalyst, uncontrolled radical polymerization was observed. The PTZ-mediated photoATRP could be temporally controlled, with no monomer conversion in the absence of irradiation,

Figure 19a. The molecular weight increased linearly with conversion, also with several "on-off" irradiation cycles,

Figure 19b. The linear semilogarithmic kinetic plot indicated a controlled radical polymerization process,

Figure 19c. Interestingly, 2-(dimethylamino)ethyl methacrylate (DMAEMA) could be polymerized with precise control over molecular weight generating polymers with low dispersity ( 1.1) under PTZ-mediated photoATRP conditions. In contrast, Ir-based photoATRP of 
DMAEMA only provided an uncontrolled polymerization due to formation of a radical cation from the amine moiety in the monomer by the oxidized $\mathrm{Ir}^{\mathrm{IV}}$ complex.

\section{Insert Figure 18.}

\section{Insert Figure 19.}

Perylene was also reported as an organic photoredox catalyst for a metal-free photoATRP of methyl methacrylate and other functionalized vinyl monomers.[132] Perylene behaved as an activator to reduce a conventional ATRP initiator, such as EBPA, to induce radical polymerization under a visible light irradiation. However, limited initiation efficiency was determined from $2 \%$ to $40 \%$ in various solvents when catalytic amounts, 0.11 equiv to initiator, of perylene were used. Although a linear semilogarithmic kinetic plot and good temporal control by light was observed, the molecular weight decreased with conversion. This indicated that the system catalyzed by perylene partially resembled an uncontrolled radical polymerization. This could be attributed to the fact that oxidized perylene radical cation did not efficiently quench the radicals. Retention of the bromine chain end functionality was limited, which was confirmed by several chain-extension polymerizations and MALDI-TOF. This might arise because of bromine abstraction from EBPA by the propagating radical as well as other undesired radical terminations, rather than a clean deactivation pathway.

Fluorescein was recently used as metal-free catalyst for photoATRP of methyl methacrylate.[133] In the presence of trimethylamine as a sacrificial electron donor, fluorescein was involved in a reductive quenching cycle to activate the alkyl bromide and generate a propagating radical. In contrast, both PTZ and perylene-catalyzed photoATRP systems were proposed to undergo an oxidative quenching cycle, and they did not require a sacrificial electron donor. A more detailed discussion is presented in section 2.7.3. Model experiments clearly showed that control over polymerization could only be obtained in the presence of fluorescein, trimethylamine, EBPA and irradiation. A polymerization with fluorescein but without trimethylamine resulted in formation of a trace amount of uncontrolled polymer (3\% monomer conversion, $M_{\mathrm{n}}=62100, M_{\mathrm{w}} / M_{\mathrm{n}}=1.92$ ). The fluorescein-catalyzed photoATRP of MMA was investigated with various amounts of catalyst $(10 \%, 20 \%$, and $30 \% \mathrm{~mol}$ ratio to initiator) and trimethylamine,

Figure 20. All three experiments gave almost linear first-order kinetic plots, and the polymerization with higher loading of fluorescein gave slower polymerization rate. The 
increasing molecular weight with conversion supported a controlled polymerization process. However, the molecular weights for obtained PMMA were much higher than the theoretical values, indicating limited initiation efficiency. Interestingly, the initiation efficiency was significantly improved by targeting higher degrees of polymerization (DP). For example, when targeting a DP of $800,86 \%$ initiation efficiency was obtained at $39 \%$ monomer conversion. This system fulfills some criteria for a controlled polymerization process such as linear kinetic plot, increasing molecular weight and high chain end functionality. However, this system was inferior to PTZ-catalyzed photoATRP due to inherent limitations in initiation and deactivation. Very recently, a visible light mediated metal-free ATRP was developed using 5,10-diaryl-5,10dihydrophenazine derivatives (Figure 18). [134]

\section{Insert Figure 20.}

\subsubsection{Polymerization of acrylonitrile}

The PTZ-catalyzed photoATRP system was also applied to the synthesis of polyacrylonitrile and produced polymers with predictable molecular weights and relatively low dispersities.[27] In addition to 10-phenylphenothiazine, 10-(4-methoxyphenyl)- and 10-(1-naphthalenyl)phenothiazine were synthesized and successfully applied to the polymerization of acrylonitrile. PhotoATRP of acrylonitrile was conducted with EBPA as ATRP initiator and catalytic amounts of the PTZ derivatives, $10 \mathrm{~mol} \%$ to initiator. Linear semilogarithmic kinetic plots were observed for acrylonitrile polymerizations in both DMSO and DMF, while the polymerization in DMSO gave a faster polymerization than in DMF (

Figure 21a). The molecular weights increased with conversion, but at the beginning of the polymerization the molecular weight was higher than the theoretical value, indicating slow initiation (

Figure 21b). 2-Bromopropionitrile was also used as an ATRP initiator instead of EBPA for the polymerization of acrylonitrile under PTZ-catalyzed photoATRP conditions. However, the initiation efficiency was not improved. High bromine chain end functionality was confirmed by ${ }^{1} \mathrm{H}$ NMR spectra and successful chain extension of polyacrylonitrile macroinitiator. This is an especially viable method to synthesize polyacrylonitrile as a precursor for carbons for electronic 
applications since it contains no residual transition metal.[135-138] On the other hand, Cumediated ATRP of acrylonitrile usually provided polymers with very narrow MW distribution $\left(M_{\mathrm{w}} / M_{\mathrm{n}}<1.10\right) .[139,140]$

\section{Insert Figure 21.}

\subsubsection{Mechanism of metal-free ATRP}

An extensive investigation recently elucidated the mechanism of metal free PTZ-catalyzed photoATRP, especially as a comparison with Cu-mediated ATRP.[141] A series of phenothiazine derivatives, including phenothiazine, 10-methylphenothiazine 10-(4-chlorophenyl)-phenothiazine and phenyl benzo[b]phenothiazine and 9-phenylcarbazole and thianthrene were tested in metalfree photoATRP in order to investigate and identify structure-reactivity relationships. Successful polymerization of MMA was conducted with all selected catalysts in the presence of EBiB as the ATRP initiator. All compounds efficiently photoactivated the alkyl bromide to generate radicals which initiated the polymerizations. However, only $\mathrm{N}$-aryl phenothiazine mediated polymerizations gave polymers with controlled molecular weights and relatively narrow molecular weight distributions, indicating that the other compounds were not efficiently involved in the deactivation process.

Upon irradiation, excited $f a c-\left[\operatorname{Ir}(\mathrm{ppy})_{3}\right]^{*}$ and $\mathrm{Ph}-\mathrm{PTZ} *$ show strong reduction ability, $-1.73 \mathrm{~V}$ and $-2.10 \mathrm{~V}$ vs SCE, respectively (

Figure 22). The most active catalyst for $\mathrm{Cu}$-mediated ATRP is $\left[\mathrm{Cu}^{\mathrm{I}}\left(\mathrm{TPMA}^{*} 3\right)\right]^{+}\left(E^{\theta}=-0.18 \mathrm{~V}\right.$ vs SCE, TPMA*3 = tris((4-methoxy-3,5-dimethylpyridin-2-yl)methyl)-amine; note that redox potential of $\left[\mathrm{CuBr}_{2}\left(\mathrm{TPMA}^{* 3}\right)\right]$ is more negative $-0.42 \mathrm{mV}$ due to a strong halidophilicity of $\mathrm{Cu}(\mathrm{II})$ species). [142] These values suggested that both $f a c-\left[\operatorname{Ir}(\mathrm{ppy})_{3}\right]$ and Ph-PTZ have much greater reactivity for the activation of alkyl halides via an outer-sphere electron transfer (OSET) processes rather than typical inner-sphere electron transfer (ISET) in Cu-based ATRP[143, 144] The activation step was studied by laser flash photolysis (LFP) experiments. Different concentration of EBPA solutions was reacted with excited $\mathrm{Ph}^{\mathrm{PTZ}}{ }^{*}$, resulting in different lifetimes. By plotting the concentration and lifetime to Stern-Volmer equation, a rate constant 5.7 $\times 10^{9} \mathrm{M}^{-1} \mathrm{~s}^{-1}$ was obtained for the reaction between EBPA and Ph-PTZ*. 


\section{Insert Figure 22.}

The OSET activation step of a metal-free ATRP was subsequently carefully evaluated by modified Marcus theory.[145-148] Based on calculations, the ground state Ph-PTZ could not activate methyl 2-bromoisobutyrate $\left(\mathrm{MBiB}, k_{\text {act, OSET }}=1.0 \times 10^{-14} \mathrm{M}^{-1} \mathrm{~s}^{-1}\right)$, while $\mathrm{Ph}-\mathrm{PTZ} *$ reacted with $\mathrm{MBiB}$ with a very large rate constant $\left(k_{\text {act,OSET }}=5.8 \times 10^{8} \mathrm{M}^{-1} \mathrm{~s}^{-1}\right)$. This value could not be directly compared to the activation rate constant of a traditional $\mathrm{Cu}$-catalyzed ATRP due to the low quantum yield $\left(\varphi=1.3 \times 10^{-3}\right)$ for Ph-PTZ*. Therefore the effective rate constant was significantly decreased by the low lifetime of the excited state and the quantum yield.

The triplet excited state of $f a c$ - $\left[\operatorname{Ir}(\mathrm{ppy})_{3}\right]$ has a much longer lifetime (1900 ns) compared to PhPTZ (4.5 ns), which resulted in much higher quantum yield for $f a c$-[Ir(ppy $\left.)_{3}\right](\varphi=0.4)$. This could also explain why photoATRP of MMA required only 50-100 ppm of $f a c$-[ $\left[\operatorname{rr}(\mathrm{ppy})_{3}\right]$, but 1000 ppm of Ph-PTZ. A much higher proportion of the Ir complex would be involved in both activation and deactivation, while most of formed Ph-PTZ* would quickly decay back to the ground state, being unable to efficiently participate in the activation and deactivation processes.

Another interesting observation in this study was that a controlled polymerization was achieved only for compounds that exhibited a reversible cyclic voltammetry. Irreversible redox behavior was detected for 9-phenylcarbazole and thianthrene (

Figure 23), indicating that the radical cation derivatives were not stable enough to deactivate the propagating radical during a polymerization. The deactivation pathways were then investigated by both Marcus and DFT theory calculations. Five possible deactivation mechanism of MiB (methyl isobutyrate) radicals were proposed, (Error! Reference source not found.): (a) the ISET mechanism through a concerted $\mathrm{Br}$ atom transfer; b) the outer-sphere dissociative electron transfer (DET) between $\mathrm{MiB}^{\bullet}$ to $\mathrm{Ph}_{-} \mathrm{PTZ}^{\bullet+} \mathrm{Br}^{-}$, providing dissociation form of catalyst and $\mathrm{Br}^{-}$; (c) the stepwise outer-sphere electron transfer (OSET-I) from $\mathrm{MiB}^{\bullet}$ to $\mathrm{Ph}-\mathrm{PTZ}^{\bullet+} \mathrm{Br}^{-}$to form an $\mathrm{MiB}^{+}$ carbocation; (d) the outer-sphere electron transfer between $\mathrm{MiB}^{\bullet}$ and the dissociated $\mathrm{Ph}-\mathrm{PTZ}^{\bullet+}$ (OSET-II); and (e) the termolecular outer-sphere associative ET (AET-ter) from $\mathrm{Ph}-\mathrm{PTZ}^{\bullet+}$ to $\mathrm{MiB}^{\bullet}$ and $\mathrm{Br}^{-}$to form the ground-state catalyst 2 and $\mathrm{MBiB}$. 


\section{Insert Figure 23.}

Among the five possible pathways, the ISET pathway required an activation free energy of 10.5 $\mathrm{kcal} / \mathrm{mol}$. Both DET and OSET-II involved the formation of carbocation had activation free energies of 13.4 and $9.2 \mathrm{kcal} / \mathrm{mol}$, respectively, while OSET-I had the highest barrier, 13.6 $\mathrm{kcal} / \mathrm{mol}$. It was concluded that AET-ter had the lowest activation free energy, $3.9 \mathrm{kcal} / \mathrm{mol}$, indicating that it was the most favorable pathway, consistent with experimental observations. Metal-free photoATRP with an alkyl chloride initiator was not controlled, ruling out the possibility of OSET-II. Additionally, the fact that there was no formation of oligomers implied no carbocation intermediates were formed, which excluded DET, OSET-I and OSET-II pathways. Consequently, the most favorable AET-ter pathway was further supported by the calculated reaction rates. The rate of AET-ter deactivation was calculated to be $\sim 200$ times faster than the rate of radical-radical termination, while rates of other deactivation pathways (ISET, DET, and OSET-I) were much slower, or in the same order of magnitude (OSET-II) as termination.

\section{Insert Scheme 8.}

By combining all the information summarized above, an overall mechanism was proposed as an oxidative quenching cycle for PTZ-catalyzed photoATRP (Error! Reference source not found.). Excitation of ground state of $\mathrm{Ph}-\mathrm{PTZ}$ by irradiation provided the excited $\mathrm{Ph}-\mathrm{PTZ}$, which had sufficient reduction potential to reduce alkyl bromide in a dissociative electron transfer process to form a carbon-centered radical. At the same time, neutral catalyst Ph-PTZ was oxidized to the radical cation form, $\mathrm{Ph}-\mathrm{PTZ}^{\bullet+}$, which was in equilibrium with the ion pair $\mathrm{Ph}-\mathrm{PTZ}^{\bullet+} \mathrm{Br}^{-}$. The radicals initiated polymerizations before being deactivated by $\mathrm{Ph}_{-} \mathrm{PTZ}^{\bullet+}$ in a termolecular associative electron transfer, which closed the catalytic cycle to reform the catalyst and polymer chain with bromine as chain end.

\section{Insert Scheme 9.}

Several side reactions were also possible in this metal free PTZ-catalyzed photoATRP. A background reaction photoinduced homolytic cleavage of the $\mathrm{C}-\mathrm{Br}$ bond would provide carbon centered and bromine radicals which could initiate a free radical polymerization. The carbon centered radical could abstract bromine from ATRP initiator or be deactivated by $\mathrm{Ph}-\mathrm{PTZ}^{\bullet+}$ to form an active polymer chain. Although Ph-PTZ* had the ability to reduce the radical to a 
carbanion, this process should be very slow due to the low concentrations of these two active intermediates in the reaction medium.

Unlike PTZ-catalyzed photoATRP, metal-free photoATRP based on fluorescein was proposed to undergo a reductive quenching cycle (Error! Reference source not found.). [133] Upon irradiation by visible light, an excited species of fluorescein was formed and quenched by excess electron donor trimethylamine to provide the radical anion form of fluorescein and an amine radical cation. Then, the radical anion form of fluorescein reduced the $\mathrm{C}-\mathrm{Br}$ bond in the ATRP initiator, or polymer chain end, to generate the propagating radical and regenerate the catalyst in ground state. It was proposed that trialkylamine radical cation oxidized the bromide anion to bromine radical which quenched the propagating radical to form a polymer with a bromine chain end. The proposed deactivation pathway was a pure radical-radical coupling reaction, which should have very slow rate. On the other hand, the deactivation reaction between $\mathrm{Br}^{\bullet}$ and $\mathrm{P}_{\mathrm{n}}{ }^{\bullet}$ competed with bromine radical-initiated polymerization. This led to an inefficient deactivation procedure which caused the molecular weight of the formed polymers to be much higher than theoretical values as well as displaying broad molecular weight distributions.

\section{Insert Scheme 10.}

\subsection{Applications}

High level of control over block copolymer formation provided access to responsive materials that can be utilized in complex environments. Full sequential control, thus close to $100 \%$ end group fidelity, allows for the formation of multiblock copolymers, permitting to mimic the primary structure and potential function of simple proteins. The photoATRP procedures featured herein provide excellent "living" characteristics and have already been employed for formation of multiblock copolymers. An amphiphilic diblock copolymer based on a block of poly(methyl acrylate) and one of poly(solketal acrylate) that was subsequently hydrolyzed formed nanoparticles in a self-assembling process and were characterized via transmission electron microscopy (TEM) and dynamic light scattering (DLS) in water (

Figure 24). [69]

\section{Insert Figure 24.}

The sequential synthesis of a decablock copolymer with a dispersity of only 1.16 and a molecular weight of 8,500 was reported.[70] Methyl, di(ethylene glycol) ethyl ether and $t$-butyl acrylate 
were polymerized in a sequential procedure adding neither copper(II) nor ligand in between chain extension steps. Since only a $\mathrm{DP}_{\mathrm{n}}=13$ was targeted, reaction times were kept between 2 and 7 hours. Increasing reaction times was required for an increasing number of blocks. Since multiblock copolymer synthesis via photoATRP relies on the presence of a bromine, or a (pseudo)halide functionality, monomer must always be present to keep radical-radical coupling reactions at a minimum.[149] Therefore, monomer conversion was monitored via Fourier transform infrared spectroscopy (FTIR) to keep radical coupling reactions to a minimum, nevertheless a clearly visible shoulder appeared in the SEC graph with increasing number of blocks (

Figure 25). Hydrolysis of the incorporated $t$-butyl acrylate units led to formation of a $\mathrm{pH}$ and temperature responsive polymer with self-assembly properties. The hydrodynamic radii of the formed decablock copolymer particles at $\mathrm{pH}=6$ changed with temperature due to thermoresponsive poly(di(ethylene glycol) ethyl ether acrylate) blocks.

\section{Insert Figure 25.}

High molecular weight multiblock polyacrylates were also investigated.[71] Blocks of 3, 10, 25 and 100 repeating units were targeted. With increasing target $\mathrm{DP}_{\mathrm{n}}$ per block, a decrease in both polymerization rate and end group fidelity was observed, limiting the number of blocks for the multiblock copolymer targeting segments with $\mathrm{DP}_{\mathrm{n}}=100$ to 6 , whereas $\mathrm{DP}_{\mathrm{n}}=3$ allowed 11 blocks with comparable dispersity of around 1.2, in agreement with results obtained for other controlled polymerization techniques. In contrast to the previous approach, fresh $\mathrm{CuBr}_{2} / \mathrm{Me}_{6}$ TREN in DMSO was added along with every $2^{\text {nd }}\left(\mathrm{DP}_{\mathrm{n}}=25\right.$ and 100$)$ or $3^{\text {rd }}$ monomer addition, respectively, improving the degree of control as well as polymerization rates. Reported reaction times were longer in these reactions, due to a different light source and lower polymerization temperature.[67]

Recently, bifunctional initiators were used for the synthesis of $\alpha, \omega$-telechelic multiblock copolymers.[67] The use of bifunctional initiators allowed for the synthesis of copolymers with up to 19 blocks, with a bimodal molecular weight distribution $(\nexists=1.45)$, indicating more termination than with the monofunctional analogues since the number of initiating sides was doubled. Although no temperature dependence was found for the synthesis of the homopolymers, the degree of control was dependent on the temperature, with lower temperatures giving better control. Consequently, an optimized protocol allowed for the formation of a 23-block copolymer 
(11 block extensions) with target $\mathrm{DP}_{\mathrm{n}}=2$ and a dispersity of only 1.18 . The optimized protocols also allowed the preparation of undecablock copolymers (target $\mathrm{DP}_{\mathrm{n}}=100$ ) with $150,000, D=$ 1.20. Despite the good agreement between theoretical and actual molar mass, a clearly visible shoulder ( $\sim 30 \%$ peak height) was observed in the SEC trace. The symmetric nature of the telechelic polymers was demonstrated via SEC after cleavage of a polymer formed with a bifunctional initiator with a disulfide linkage, yielding a polymer with half of the initial molecular weight. A multiblock star polymer up to 21 arms was recently synthesized via photoinduced $\mathrm{Cu}$ based ATRP. [150]

Sequence-defined polymerizations were one step beyond sequence-control, providing a precise number of monomer units per block, i.e. the precise equivalent to proteins or DNA. Sequencedefined polymers could potentially be used to encode information.[151] Recently, the synthesis of sequence-defined acrylate oligomers was reported using photoinduced copper-mediated single unit monomer insertions.[152, 153] Starting from EBiB, methyl acrylate and up to 4 other acrylate monomer units were sequentially inserted into the copolymer. The conversion was monitored in situ via FTIR spectroscopy. After each monomer insertion step, the desired products were isolated via preparative recycling SEC. Interestingly, chlorine end groups were identified as unreactive side products after purification, which could be linked to presence of traces of chlorinated solvents during the photoreaction.

Synthesis of molecularly imprinted polymer (MIP) nanoparticles was reported, which recognized testosterone and $\beta$-propanolol, based on photoATRP processes using an iridium catalyst.[154] The MIPs were crosslinked polymer matrices formed in the presence of a template molecule. The template molecules were removed after the polymerization and left a cavity that preferentially binds the template molecule. PhotoATRP was a beneficial procedure for MIP formation as it could be performed at low temperatures. The controlled nature of the network formation enhanced molecular recognition by providing more homogenous recognition sites. Iridium catalysts were chosen to ensure compatibility with acidic monomers, which were required to reach good imprinting.

One of the biggest constraints of photoreactions is the low illumination efficiency arising from the optical density of the reaction mixture. Efficient exposure to light is only given for the outermost layers of a reaction container, as strong photo-gradients were imposed by BeerLambert's law. To overcome this limitation, photoreactions can be carried out in continuous flow reactors that provided shorter path lengths compared to batch reactors, [155] and which provide 
scalability of polymerizations by increasing tube lengths and parallelization of reactors. Homogeneous reaction conditions as well as scalability and more efficient heat release are some additional advantages of flow reactors.[156] The significant impact of efficient illumination in flow reactors is best visualized by the speed-up of otherwise very slow [2+2] cycloadditions, which can be accelerated from several hours to a minute reaction time when switching from batch to flow operation.[157, 158] PhotoATRP in a continuous flow reactor for methyl and $n$-butyl acrylate was reported with $\mathrm{CuBr}_{2} / 6 \mathrm{Me}_{6}$ TREN in DMSO.[86] The reaction was carried out at $15^{\circ} \mathrm{C}$ in microreactors. Good control over the molecular weight distribution was obtained along with a speed-up by a factor of 4 compared to (small scale) batch systems. Very high end group fidelity was demonstrated by ESI-MS and underpinned by block copolymer formation. More recently, MMA was polymerized in a flow reactor. PMDETA was selected as ligand in order to attain good block structures.[75] In this case the reaction was around 7 times faster than in batch. The strongest increase in polymerization rate of a photopolymerization reaction so far was observed for Co-mediated photoCMRP.[159] Remarkably, control was also maintained for UV irradiation in flow above room temperature, whereas crosslinked polymers were found for the same reaction in batch systems. Recently, Ir-mediated flow photoATRP was reported for the synthesis of PMMA, demonstrating similar advantages for flow reactions over batch processing.[160] As mentioned above, rate increase and avoidance of side reactions caused by too high light intensities directly at the reactor wall, is one advantage of using flow reactors. However, highest potential is the simple scalability of the reactions. While batch photoreactions become increasingly inefficient when increasing reactor dimensions, flow reactors are easily scaled up while maintaining short optical pathlengths. Flow reactors are hence ideal to produce larger amounts of material compared to batch, where often only a few grams are synthesized.

\section{Photomediated RAFT polymerization}

The inherent difficulty in performing photoRAFT resided in the fact that RAFT agents are typically good UV-absorbers and are therefore prone to UV-degradation. Conditions must be chosen to allow for initiation of the polymerization while retaining the integrity of the RAFT end group functionalities. Several strategies are discussed in the following sections, starting with the so-called iniferter polymerization mechanism. Photoinduced iniferter reactions can be seen as precursor technique of RAFT as some features between both techniques are similar. Iniferter initiation processes played an important role in the development of modern photo RAFT procedures and are thus described first. 


\subsection{Iniferter polymerization}

Iniferter (initiator-transfer agent-terminator) polymerization is among the oldest living/controlled radical polymerization techniques.[161,162] An iniferter agent is defined as a molecule that can decompose into radical fragments to initiate chain growth. Iniferter-derived radicals were thereby expected to end chain growth by end-capping the growing chains. This end-capping was either reversible, or to be prone to transfer reactions. The iniferter technique was followed by a photoinitiated iniferter method. In a photoiniferter polymerization a UV-labile bond is homolytically cleaved, followed by polymerization and end-capping of polymer chains. The polymers, containing specific thioether or thioester end groups, can be reactivated by UV-light, effectively establishing a reversible termination equilibrium. Dithiocarbamates form one class of photoiniferter agents, but could also act as RAFT agents. Dithiocarbamates are good control agents for vinyl acetate RAFT polymerizations.[163] Vice versa, classical RAFT agents, such as dithiobenzoates and trithiocarbonates can in principle also act as RAFT mediators and concomitantly as photoiniferters (see below).

Photoiniferter reactions can be considered as the first example of a successful photoRDRP process. Polymerization of a variety of monomers was possible, including styrene, acrylates, methacrylates, vinyl esters and derivatives thereof, however with varying levels of success.[161, 164-167] As mentioned above, dithiocarbamates were most commonly used but dithiols, selenides or phosphines could also act as photoiniferters.[162, 164, 167-171] The recently developed nitroxide-mediated photopolymerization can also be classified as a photoiniferter process.[172] Complex block copolymer architectures have been reported for classical photoiniferter polymerization.[166, 173-175]

\subsection{RAFT initiated by conventional photoinitiators}

RAFT polymerizations can be initiated by conventional PIs. Most dithioesters are colored and absorb light in the range of ca. 500-600 nm. Hence, even under visible light irradiation, decomposition of the RAFT agent may occur, rendering photoRAFT polymerization reactions with these transfer agents difficult. Trithiocarbonates are typically blue-shifted, and hence absorb in the region below 400-450 $\mathrm{nm}$. Still, trithiocarbonates decompose under UV light, but usually to lesser extent and are thus a better choice for any photoRAFT reaction.

The first photoRAFT polymerization was demonstrated in 2002.[176] Two different dithiobenzoates were employed to control styrene polymerization. Initially, well controlled polymers were obtained, $(\nexists<1.2)$, however only at $<30 \%$ monomer conversion, thus short 
irradiation times. When extending the reaction time to higher conversions, discoloration of the RAFT polymer was observed, accompanied by broadening of the molecular weight distributions. The effect was attributed to the loss of RAFT agent in secondary decomposition reactions after homolytic cleavage of the C-S bond. Interestingly, no external photoinitiator was employed, and hence the polymerization relied on initiation of the reaction by the iniferter mechanism (see section 3.3).

Later, trithiocarbonate, specifically DDMAT, $S$-dodecyl-S'-( $\alpha, \alpha$ '-dimethyl- $\alpha$ ' '-acetic acid, was used for photoRAFT reactions.[177] When exposed to longer wavelength UV light, > $365 \mathrm{~nm}$, only a few percent of the RAFT agent decomposed even after irradiation over a few hours. With this RAFT agent, in combination with a conventional PI (2,4,6-trimethylbenzoyl)diphenyl phosphine (TPO), poly(methyl acrylate) was obtained up to $90 \%$ conversion with dispersity below 1.1 and with an excellent linear correlation between $M_{\mathrm{n}}$ and conversion. Block extension and reactivation of the residual macro RAFT agents were presented, underpinning that if the wavelength regions of the RAFT agent and the PI were chosen appropriately, polymerizations could be efficiently performed.[178] This approach was used in kinetic studies,[179-182] via socalled pulsed laser polymerization experiments, and propagation and termination kinetics of macroradicals could be assessed. The RAFT process was employed to probe chain-length dependencies, especially in the termination reaction. In these experiments, laser generated populations of initiating radicals rapidly enter the main equilibrium of the RAFT process, hence converting all radicals into macroradicals with a specific chain length.[181, 183] Later this concept of pulse laser-induced RAFT polymerization was extended to study RAFT kinetics.[184190]

PI RAFT reactions have successfully been applied to numerous monomers. The outcomes of polymerizations under UV and/or visible light irradiation were comparable to results obtained under thermal polymerization conditions, but also under mild reaction conditions. This was especially interesting for preparation of polymers with distinct lower critical solution temperature (LCST) behavior. 
Table 5 gives an overview of the selection of photoRAFT reactions reported in literature. In almost all cases, trithiocarbonates were chosen over dithiobenzoates for the reasons discussed above. Light sources varied largely, with a trend towards visible light irradiation with a full irradiation spectrum or blue and green light. In general, when UV light in the range of $365 \mathrm{~nm}$ was employed, a superimposition of the typical RAFT mechanism with the iniferter reversible termination mechanism could be postulated (see section 3.3 for details). A distinction between both mechanisms was difficult since product were identical for both mechanisms. Little information could be gathered from the typical tests of controlled polymerization. The presence of reversible termination iniferter reactions was indicated by a dependence of the overall polymerization rate on the concentration of the RAFT agent. Increasing $c_{\text {RAFT }}$ resulted in increasing rate, which could only be explained on the basis of the iniferter mechanism. Obviously, the higher the wavelength employed for the light source, the less influence was from iniferter reactions.

Homo- and block copolymers were synthesized such as polystyrene, PS, polystyrene) $b$-poly $(\alpha-$ vinylbenzyl alcohol) PS- $b$-PVBA[191] and poly(butyl acrylate), PBA[192] using the RAFT agent S,S'-bis( $\alpha, \alpha$ '-dimethyl- $\alpha$ '-acetic acid), BDMAT. Poly(glycidyl methacrylate), PGMA, and poly(butyl methacrylate), PBMA and block copolymers were interestingly polymerized with a dithiobenzoate, namely 2-cyanoprop-2-yl(4-fluoro) dithiobenzoates, CPFDB.[193] Even when a broadband irradiation source was used, no breakdown of the RAFT agent was observed on the timescale of the polymerization. Block copolymerizations were targeted with visible light. The ethyl substituted derivative of DDMAT was used to obtain water-soluble polymers based on poly( $N$-(2-acryloyloxyethyl) pyrrolidone) (PNAP) and poly(poly(ethylene glycol) acrylate) (PPEGA) directly by reaction in water as reaction medium. [194] Another water-soluble polymer, poly( $N$-isopropyl acrylamide) (PNIPAAm), was obtained using BDMAT and Irgacure 2959 as PI in DMF solution.[195] Also, vinyl acetate was polymerized using xanthates.[196, 197] With the xanthate methyl(ethoxycabonothioyl)sulfanyl acetate (MESA), poly(vinyl acetate) (PVAc) with well controlled molecular weight was obtained. Interestingly, some degree of stereocontrol could also be achieved as the solvent and polymerization temperature had an influence over tacticity. In this way, stereoblock copolymers were synthesized.

\section{Insert Table 5.}




\subsection{Initiator-free photoRAFT}

As described above, contributions from iniferter initiation and reversible termination to the PIinduced photoRAFT reactions were commonly observed, even if the mechanistic situation was somewhat ambiguous regarding quantification of the effects.

When RAFT agent is continuously decomposed under UV-incidence but only to a low extent, the radicals produced from the iniferter mechanism can maintain the reaction, essentially leaving the RAFT equilibrium unaffected. Error! Reference source not found. describes this case. The RAFT mechanism with its pre- and main equilibrium remained fully active and was unaltered compared to a conventional RAFT process. The only difference is that the initial RAFT agent could serve as a radical source by homolytic C-S bond cleavage. The thiyl radical that is at that instance created can trap propagating macroradicals, yielding a macroRAFT agent that takes part in the main RAFT equilibrium. They can also dimerize forming thiuram. The $\mathrm{R}$ group radicals initiate chain growth and act as a conventional initiator. Once all initial RAFT agent is consumed by the pre-equilibrium (composition plays only a minor role with regards to RAFT agent conversion), radicals are generated by the reversible termination action of the macroRAFT. A beneficial side-effect of this process is that all residual chains (with the exception of chains stemming from bimolecular termination) carry not only the desired trithiocarbonate (TTC) end group in $\omega$-position, but also the $\mathrm{R}$ group in $\alpha$-position. Hence, polymers are more pure than thermally initiated RAFT polymers, or polymers obtained from PI-initiated photoRAFT, where a fraction of initiator-derived radicals is always found in the polymer as a side product. A similar approach was previously reported for concurrent ATRP/RAFT process.[198-200]

\section{Insert Scheme 11.}

As with the PI-induced RAFT reactions, trithiocarbonates are preferred for initiator-free photoRAFT reactions, as decomposition should be kept low to reduce consumption of the RAFT agent in secondary decomposition reactions. The average radical concentrations must also be kept low in order to prevent irreversible bimolecular termination. Therefore a UV light source is preferred over visible light, as trithiocarbonates absorb typically between 350 and $400 \mathrm{~nm}$.[178] For example, poly(acrylic acid) (PAA) was obtained with different molecular weight directly from polymerization in water at room temperature by reaction with BDMAT, achieving polymers in the molecular weight range of 3,000 to 40,000 with dispersities below 1.1.[201] The same principle was applied to polymerization of styrene. However, instead of a conventional TTC 
RAFT agent, the complexity of agent was increased by polymerizing a bifunctional TTC with dichloro-p-xylene, which yielded a polymer with a molar mass of few thousand Da with several TTC moieties built into the main chain. Polymerization of such polyTTC directly yielded multisegmented polymer materials, which could be later degraded into their single segments by aminolysis. These results show that no significant difference exists between a thermal RAFT process (employing then a thermal initiator) and an initiator-free photoRAFT reaction. Segment growth of the PS units was uniform, well controlled and could be reinitiated after further monomer addition, yielding polymers of up to 30,000 with each segment of ca. 4,500 ( $\oplus$ =1.28).[202] Using the same RAFT agent, the polymerization of PBA and PS was achieved.[203] Both monomers were successfully polymerized with very good match between theoretical and experimental molecular weight. Since BDMAT is a symmetrical TTC, chains grew to either side of the RAFT agent. Consequently, chain extension led to formation of triblock copolymers. In this manner, high molecular weight PS- $\boldsymbol{b}$-PBA- $\boldsymbol{b}$-PS polymer with $\mathrm{M}_{\mathrm{n}}>200,000$ was obtained (see 
Table 6 for a collection of examples of initiator-free photoRAFT polymerizations). DFT calculations were performed on the bond dissociation energy of the C-S bond, showing that the tertiary nature of the leaving group of BDMAT was essential for the process. If the R group was replaced by a less stabilized radical fragment, less control was achieved over the RAFT polymerizations, which was also confirmed by experiment.[204] PVAc was obtained using xanthates as mediators/initiators.[205] Similar to the phenylbis(2,4,6-trimethylbenzoyl)phosphine oxide (BAPO)-initiated polymerizations ( 
Table 5), well controlled polymerizations were observed with a polymerization rate increasing with increasing xanthate concentration. To facilitate decomposition and re-initiation of the $\mathrm{R}$ group, a specific derivative of MESA was employed, $S$-2-cyano-2-propyl- $O$-ethyl xanthate (CPEC), which decomposed to yield a cyanoisopropyl fragment, as would normally be obtained from AIBN. Polymerizations without BAPO were slower, but still faster than their thermal counterparts.

In a detailed study on the kinetics of initiator-free photoRAFT, the effect of RAFT agent concentration (DDMAT) on methyl acrylate polymerization was examined, alongside a detailed study on the effect of increasing light intensities.[206] In-situ NMR measurements further confirmed the suggested mechanism, shown in Error! Reference source not found.. Higher light intensities led to increased radical concentrations and hence acceleration of polymerization.

Figure 26 displays the fourfold rate increase in rate of polymerization when going from 3 $\mathrm{mW} \cdot \mathrm{cm}^{-2}$ to $48 \mathrm{~mW} \cdot \mathrm{cm}^{-2}$. It is noteworthy that the intensity increase showed no negative effect on the polymerization. In all cases polymer of almost identical molecular weight and dispersity was obtained.

\section{Insert Figure 26.}

Interestingly, the iniferter and RAFT mechanism could also be decoupled.[207] Starting from a xanthate-terminated poly ( $N$-vinyl carbazole) (PNVP) or xanthate-terminated PVAc, the iniferter reaction was used to initiate chain growth with acrylic crosslinkers. As xanthates are not efficient mediators for activated monomers, no RAFT equilibrium was established. Rapid crosslinking occurred, showing that RAFT end groups could also generally serve as efficient macroinitiators for free radical polymerizations.

Whether TTCs could be activated under visible light conditions was then investigated.[208] In fact the excited $\pi^{*}$ state can also be reached by absorption in the blue light region when TTCs were employed. Consequently, polymers could be obtained by irradiation with blue LED. Polymers with pristine end group fidelity were obtained, which was further exemplified by multiple chain extensions of the RAFT polymer. Hexablock amphiphilic block copolymers were obtained using this process, thus confirming a high end group fidelity. Results from polymerizations from this study are summarized in 
Table 6. The potential of initiator-free photoRAFT was further explored for precision polymer design (

Figure 27).[209] Very pure star polymers could be obtained based on PMA polymers made via DDMAT, with variation in the number of arms depending on the length of the PMA building block and crosslinker concentration. Polymeric star nanoparticles were obtained with molecular weights ranging from roughly 55,000 (7 arms, $Ð=1.59)$ to $38,450,000 \quad(\sim 720$ arms, $\oslash=1.94)$. The reshuffling of trithiocarbonates and also thiocarbamates was successfully applied to prepare self-healing materials under UV and also visible light conditions under oxygen-free and ambient conditions.[210, 211]

\section{Insert Table 6.}

\section{Insert Figure 27.}

Recently, visible light mediated photolysis RAFT agent was applied under a catalyst-free photoRAFT conditions using blue and green light.[212] It was further extended to a polymerization-induced self-assembly (PISA) system. [213]

As described above, good polymerization results were achieved for PI-free as well as PI-initiated photoRAFT polymerizations. Polymers with similar structures were prepared with a slight advantage of PI-free photoRAFT, since no PI fragments would incorporate as the end group in the $\alpha$-position of a fraction of the chains. An important difference that did exist, however, was reaction rate. Photoinitiator-free photoRAFT scaled in overall polymerization rate with the initial RAFT agent concentration, while PI-initiated photoRAFT scaled with PI concentration. Consequently, photoRAFT reactions in absence of PIs typically proceeded on the timescale of several hours to tens of hours, while PI-initiated photoRAFT often proceeded within tens of minutes to only few hours reaction time. The only exception was vinyl acetate polymerization mediated by xanthates. VAc polymerized even in absence of a PI within one hour, with PI reactions running to completion in only a few minutes. The long reaction times in the PI-free scenario therefore often limit the achievable conversion and many examples discussed above were stopped at conversions below $50 \%$. 


\subsection{Photoinduced electron transfer (PET)-RAFT}

An extension to the PI-induced RAFT concept was recently introduced, which was termed photoinduced electron transfer (PET)-RAFT polymerization.[214] Inspired by the work of Irmediated photoATRP, $[119,215]$ photoredox catalysts were used to initiate a polymerization, which then proceeded via a classical RAFT polymerization pathway.

PET-RAFT can be classified as an efficient PI-induced photoRAFT process, as it featured similarities with PI-free photoRAFT. Kinetically, the reaction is driven by the metal complex concentration (ppm range). From a product point of view, no PET-initiator is incorporated into the polymer product. Thus, PET-RAFT combines the advantages of the above described photoRAFT systems. Moreover, the use of metal complexes for initiation allows the procedure to work with materials that absorb in the visible light region, hence giving the possibility to completely decouple the RAFT process from any background-iniferter or RAFT agent decomposition reactions. Consequently, reactions proceeded comparatively fast (the system given in Error! Reference source not found. was not optimized for rate) but later PET-RAFT systems allowed for reaction times in the order of just few hours. End group fidelity wags high. This system resembles concurrent ATRP/RAFT systems (without irradiation). [198-200]

\section{Insert Scheme 12.}

In the initial paper,[214] PET-RAFT was shown to be highly versatile, demonstrating the successful photo-controlled polymerization of MMA, MA, tBuA, St, $N, N$-dimethylacrylamide, $N$ (2-hydroxypropyl) methacrylamide (HPMA), NIPAAm, oligo(ethylene glycol) methyl ether methacrylate (OEGMA), OEGA, isoprene, vinyl acetate, vinyl pivalate (VP), NVP and dimethyl vinylphosphonate (DVP). Trithiocarbonates were used for activated monomers; while xanthates were used for non-activated ones, Error! Reference source not found.. Polymerizations proceeded in a well-controlled manner, and up to decablock copolymers were achieved.

Follow-up research significantly broadened the application of PET-RAFT. Polymerizations were largely independent of oxygen content, and a similar degree of livingness of polymerizations was observed in presence or absence of air ( 80\%).[216] This feature also allowed for the synthesis of a variety of quasi-pentablock copolymers by sequential addition of monomers without purification steps in between chain extensions.[217] Using a ruthenium catalyst $\left(\mathrm{Ru}(\mathrm{bpy})_{3}\right)$, similar oxygen tolerance could be observed, leading to well-defined polymer products and synthesis of decablock copolymers.[218] In a further study, PET-RAFT conditions were 
combined with Lewis acid mediators that influence tacticity of polymers, by which stereoblock copolymers could be obtained.[219]

Insert Table 7.

PET-RAFT, similar to photoATRP, is not necessarily dependent on metal-based catalysts and organocatalysts are able to induce a PET process. Thus, efficient PET-RAFT reactions were demonstrated using eosin Y,[220] donor-acceptor porphyrins[221] or even chlorophyll.[222] In all three cases, good control over polymerization was achieved, leading to different polymers, see 
Table 7 for examples. Porphyrins were combined with metals in order to tune the absorption wavelength over the whole visible light spectrum.[223] PET-RAFT was already adopted for use under the name of conventional PI-induced photoRAFT. Curcumin was demonstrated as an efficient PET-RAFT catalyst.[224] $\mathrm{Nb}(\mathrm{OH})_{5}$ nanoparticles were also used as initiators, as well as dimanganese decacarbonyl $\mathrm{Mn}_{2}(\mathrm{CO})_{10},[225,226]$ and 10-phenylphenothiazine (PTZ) as organocatalyst.[227]

PET-RAFT system was extended to conduct the polymerization by near-infrared/far-red irradiation.[228, 229]1 It was especially useful to polymerize some photosensitive monomers such as $o$-nitrobenzyl methacrylate.[230] A selective activation of RAFT agents under different wavelength was reported, achieving single monomer addition. [231]

\subsection{Applications}

Despite being a relatively recent field of research, photoRAFT in its different variations was applied in a number of different applications, with a strong focus on crosslinked materials and (nano)particle synthesis. Examples of various applications are given in the following section.

\subsubsection{Advanced photoRAFT}

As discussed in section 2.8, continuous flow photoreactors could avoid several obstacles in photoinduced reactions.[155] The RAFT/iniferter reaction of a symmetrical trithiocarbonate[232] was significantly faster in a Booker-Milburn designed flow reactor compared to batch reactions.[233] Within one hour, high monomer conversions were reached for a series of acrylates and acrylamides, with dispersities below 1.2, allowing the synthesis of PDMA with molecule weights above 100,000 while batch reactions require much more extended reaction times. Photoinduced RAFT of (meth)acrylates and acrylamides was conducted in a tubular continuous flow reactor with Irgacure 369 and Irgacure 819 at ambient temperature. [234]

\subsubsection{Particle synthesis}

In the initial photoRAFT dispersion polymerization, styrene was polymerized in the presence or absence of AIBN with a dithiobenzoates CTA.[235] The dithiobenzoates carried a carboxy moiety on the R-group to make the agent surface active. The suspension was stabilized by poly(vinylpyrrolidone). The typical behavior of an initiator-free photoRAFT polymerization was observed; increasing rate with increasing RAFT agent concentration, but interestingly no large variation in rate when AIBN was added. This could be attributed to the low efficiency of AIBN as photoinitiator. While control over molecular weight was lower than in a solution polymerization 
and dispersity was in the range of 2, overall satisfying results were obtained, demonstrating that photoRAFT was applicable to dispersed systems. An improvement of the dispersion system was reported with sodium dodecyl sulfate (SDS) as surfactant. The RAFT agent used was a surfmer, coined surfiniferter.[236] A well-controlled PMMA was obtained via emulsion approach, with molecular weights in the range of 400,000 and dispersities around 1.3.

A dispersion polymerization was employed to produce close to monodisperse microspheres from PI-initiate photoRAFT polymerizations of MMA.[237-239] The use of a trithiocarbonate (BDMAT) allowed preparation of monodisperse particles in a one-stage procedure by delaying the initial polymerization rate during the nucleation phase. Polymers followed a controlled polymerization pathway and could be reactivated after addition of fresh monomer,

Figure 28. In this way, a variety of functional monomers could be introduced to form functional surfaces on the microspheres, including poly(vinylpyrrolidone) or HPMA. This work also presented a pathway towards synthesis of functional microspheres by using hydrophilic macroRAFT agents as surfmer to stabilize the particles.[240] Various macroRAFT agents based on the TTC DDMAT and PEGA, AA and 4-vinyl pyridine were prepared, for example the microspheres containing AA on the surface showed pH-responsive behavior. Subsequently, variations of core-shell microsphere particles were synthesized, e.g. particles with thermoresponsive properties based on PEGA and methoxyethyl acrylate.[241] The concept was also suitable for synthesis of metal-composite particles,[238, 240] or particles suitable for bioconjugation via 1-ethyl-3-(3-dimethylaminopropyl)carbodiimide (EDC) coupling.[242] In a similar procedure monodisperse microparticles were obtained by direct photoRAFT of crosslinking monomers.[243]

\section{Insert Figure 28.}

Miniemulsion polymerization has also been addressed in addition to suspension and conventional emulsion polymerization, by employing a PET-RAFT process. In the controlled polymerizations of styrene using the $\operatorname{Ir}(\mathrm{ppy})_{3}$ catalyst system, good temporal control was achieved and characteristics of controlled polymerization was retained.[244]

Nanoparticle synthesis was reported using polymerization-induced self-assembly (PISA) procedure.[245, 246] MacroRAFT agents based on oligo(ethylene glycol) acrylate were chain 
extended in a PET-RAFT process with a Ru(bpy) ${ }_{3}$ catalyst under blue light irradiation. Selfassembly was induced by chain extension with benzyl acrylate. Depending on catalyst concentrations (

Figure 29), different particle morphologies were obtained. While the catalyst had no direct influence on individual block lengths, it altered the rate of polymerization and hence longer benzyl acrylate blocks were obtained with higher catalyst loadings. Overall, spherical micelles, vesicles and worm-like micelles could be obtained by this method simply by variation of solid content and catalyst concentration.

\section{Insert Figure 29.}

\subsubsection{Synthesis of gels and networks}

PhotoRAFT reactions were applied to the synthesis of networks in the bulk phase. Dental resins became more stress-resistant when RAFT agents were added to the photopolymerization formulation. The RAFT agent regulated the segment length between two crosslinks, altering the strength of the network.[247] Post-polymerization modifications became possible when the networks were formed in which the typical RAFT groups were incorporated into the main chain rather than being attached at the end of chain branches. A polymer gel synthesized from monomer and a trithiocarbonate-containing crosslinker monomer did not only lead to a controlled network polymerization, but also allowed control over the mesh size in the network after polymerization, simply by irradiating the gels with UV light.[248] The C-S bonds were reversibly cleaved by the action of the UV light, giving the network the chance to rearrange depending on the external circumstances, in a quasi-self-healing process. In a similar approach, a symmetrical RAFT agent with dienophile end groups could form crosslinks in tetrazine Diels-Alder reactions. The polymerization could be carried out following the iniferter initiation mechanism. Also, a reshuffling of the network structure and chain extension of the network segments became accessible by light irradiation in a post-polymerization process when fresh monomer was present.[249, 250] 


\section{Photomediated NMP}

Nitroxide-mediated radical polymerization (NMP) is one of the most common CRP techniques that employs reversible thermal homolysis of $\mathrm{C}-\mathrm{ON}$ bond of an alkoxyamine to produce an initiating radical and a persistent radical, a nitroxide. The conventional NMP requires high temperatures and long polymerization times for the activation of strong oxygen-carbon bonds present in the dormant species. The controlled polymerization of meth(acrylate)s is rather difficult due to their high equilibrium constants. The slow recombination of the nitroxide and the sterically-hindered chain-end radicals lead to high concentration of active radicals that promote the formation of "dead" polymers via self-termination at low conversions. This limitation has been partly compensated by recently developed alkoxyamines having higher equilibrium constants at lower temperatures thereby providing acceptable rate of polymerization for meth(acrylate)s. However, the use of the second generation alkoxyamines in NMP still require a high temperature, such as heating at $90{ }^{\circ} \mathrm{C}$. Photosensitive alkoxyamines that generate reactive species by light irradiation have been recently introduced to mediate controlled radical polymerization at ambient temperatures. The initial work on photoinduced NMP was based on the homolysis of TEMPO (2,2,6,6-tetramethylpiperidinyloxy-4-yl radical)-based alkoxyamines.[251] In this case, photochemical activation of TEMPO derivatives containing either xanthone or pyrene moieties generated reactive carbon-centered radicals and stable TEMPO radicals. Although the photosensitization step was successful, these molecules could not be used in polymerization reactions due to the low efficiency in generating the carbon-centered radicals.[252, 253] After these initial results, new photosensitive alkoxyamines bearing different chromophores covalently linked to the TEMPO-OH moiety were designed. Some successful polymerizations were reported using these compounds but either uncontrolled polymers with much higher molecular weights and broader molecular weight distribution or very low monomer conversion were also obtained ( 
Table 8).

Quite recently, new second generation alkoxyamines bearing photosensitive benzophenone, quinolone or xanthone groups were developed to accomplish NMP under more ambient conditions.[172] In addition to experimental studies on the influence of the types and positions of chromophore as well as nitroxide groups on the NMP process, theoretical studies were extensively investigated.[254-257] In the first step, the photosensitive alkoxyamine generated an active alkyl and a stable nitroxide radical under UV light (

Figure 30). The alkyl radicals could initiate polymerization, whereas the nitroxide radicals were responsible for reversibly deactivating the propagating radicals, establishing a rapid equilibrium between active and dormant species. In order to enhance the energy transfer as well as generation of alkyl radicals, the chromophore groups were deliberately located close to the aminoxyl function, 1 or 2 carbons, which was responsible for the cleavage of the $\mathrm{C}-\mathrm{O}$ bond. Faster polymerization rates, $80 \%$ conversion in $500 \mathrm{~s}$. for NMP of n-butyl acrylate were observed in addition to partial living characteristics, as suggested by the close agreement between experimental and theoretical molecular weights and the relatively low molecular weight distributions.

\section{Insert Figure 30.}

Notably, these photosensitive alkoxyamines could potentially act as a bimolecular photoinitiator in combination with a co-initiator, e.g. alcohols, amines or thiols, and produce two different radicals during irradiation. Some of these radicals might contribute to an independent initiation resulting in a polymerization that proceeds in an uncontrolled manner. The photosensitive alkoxyamines could be also efficiently direct the thermally induced NMP process by heating at $120{ }^{\circ} \mathrm{C}$. Using these two different NMP modes, the thermal polymerization of styrene at $120{ }^{\circ} \mathrm{C}$ and sequential photopolymerization of isobornyl acrylate, block copolymers with moderate molecular characteristics were obtained.[258] In a recent study, photosensitive nitroxides bearing different chromophore groups (benzophenone, naphthalene and quinoline) were developed for the photoinduced NMP of methyl methacrylate in the presence of 2,2-dimethoxy-2-phenyl acetophenone as photoinitiator.[58] In contrast to linear polymer growth with narrow molecular weight distributions (1.3-1.4), a loss of end-group functionality was confirmed by a chain extension study. 


\section{Insert Table 8.}

\section{Other Controlled Radical Polymerizations}

\subsection{Photomediated CMRP}

The use of organocobalt(III) compounds in cobalt-mediated radical polymerization (CMPR) is receiving growing attention because of the reversible formation of a carbon-cobalt covalent bond under mild conditions.[259, 260] Since the Co-C bond can be easily cleaved by heat or light, CMPR was successfully applied for various monomers such as vinyl acetates, acrylates and acrylamides.[261] In earlier studies, UV light not only facilitated the generation of initiating radicals from photosensitive alkyl cobaloxime compounds but also manipulated the reversible cleavage of the $\mathrm{Co}-\mathrm{C}$ bond throughout the polymerization.[262] However, the scope of the cobaloxime compounds was limited to acrylates. The use of bis-acetylacetonatocobalt complex $\left(\mathrm{Co}(\mathrm{acac})_{2}\right)$ in CMRP not only broadened the range of monomers but also allowed the polymerization to be conducted at lower temperatures, even below room temperature.[263] The photoinitiated version of CMRP using $\mathrm{Co}^{\mathrm{II}}(\mathrm{acac})_{2}$ and UV irradiation of a typical azo-initiator provided well-defined polymers with very high molar masses. Subsequently, this method was further extended by using commercially available photoinitiators, such as 1,2-diphenyl-2,2dimethoxyethanone and bis(2,4,6-trimethylbenzoyl)phenylphosphine oxide. The photodissociation of organocobalt(III) compounds into an organic radical and a cobalt(II) metalcentered radical upon exposure to UV or visible light was confirmed by both spin-trapping and kinetics of polymerization experiments. The Co-C bond was highly photosensitive so that the polymerization only occurred under light irradiation when the light source turns off, polymerization was immediately stopped. Furthermore, the polymerization kinetics could be tuned by photoinitiator type, its concentration and irradiation time, which directly affected the initiating efficiency of the system.

Recently, organocobalt porphyrin derivatives have been developed for thermally and photochemically initiated CMRP to eliminate the need to use conventional radical initiators.[264] By applying a visible light source or sunlight to the organocobalt porphyrin, a number of structurally different monomers, both hydrophilic and hydrophobic, could be successfully polymerized in a controlled manner. Some key features of this system included mild reaction conditions and no need for additional compounds such as dyes, photosensitizers, or reducing agents. The organocobalt porphyrins were highly photosensitive so that the visible light irradiation could facilitate mediation of chain growth by reversibly activating the $\mathrm{Co}-\mathrm{C}$ bond ( 
Figure 31). A series of well-defined multi-block copolymers containing acrylate and acrylamide segments were prepared via photoinduced CMRP using a simple household light source or sunlight.[265] Furthermore, this system provided excellent temporal control over the chaingrowth process, enabling the synthesis of complex macromolecular structures.

\section{Insert Figure 31.}

Structurally similar organocobalt salen complexes (salen is a common abbreviation for $\mathrm{N}_{2} \mathrm{O}_{2}$ bisSchiff base bis-phenolates) were also used as control agents for the polymerization of acrylates, acrylamides and vinyl acetate, under ambient conditions, i.e. visible light irradiation at room temperature.[266] Since the organocobalt salen complex has a relatively weak $\mathrm{Co}-\mathrm{C}$ bond, the direct photolysis of this complex generates an organic radical that can initiate polymerization and a persistent metal-centered radical that is responsible for the reversible termination of the radical on the growing polymer chain end. Because of its convenient synthesis, just mixing commercial available cobalt(II) salen complexes with a traditional photoinitiator, this system was more suitable for industrial applications, compared to organocobalt porphyrin systems.[267] This system enabled the preparation of well-defined homopolymers and copolymers with predetermined molecular weight and narrow molecular weight distributions under mild conditions.

The photomediated CMRP process was recently implemented in continuous flow reactors using $\left(\mathrm{Co}(\mathrm{acac})_{2}\right)$ complex with UV light irradiation.[159] The flow reactor process not only led to a significant improvement in polymerization rates, but also allowed scalable synthesis of polymers by simplifying purification steps. This process was successfully applied to the polymerization of vinyl acetate and its copolymerization with a less reactive olefin 1-octene to obtain random copolymers containing up to $50 \mathrm{~mol} \%$ of 1-octene. This continuous photoflow system enabled reaching high conversion in several polymerization processes by increasing the light intensity and efficiency that reduced reaction times from days to minutes.

\subsection{Photomediated ITP}

Iodine transfer polymerization (ITP) was one of the earliest CRP methods and is based on the cleavage of C-I bond via a degenerative chain transfer mechanism.[268] The method was particularly suitable for the polymerization of fluorinated and phosphonic monomers and was used industrially to produce high value thermoplastic elastomers based on hard-soft-hard 
triblock copolymers. The basic ITP system consisted of a monomer, an iodinated chain transfer agent and a conventional initiator, but did not comprise any metal. Due to the weak C-I bond, the iodinated chain transfer agents were very sensitive to both heat and light, and tended to decompose during synthesis, isolation, or storage. To overcome these limitations, the required iodinated compounds could be formed in situ from a free radical initiator in conjunction with iodine $\left(\mathrm{I}_{2}\right)$. Conventional thermal initiators such as BPO, AIBN and potassium persulfate were mainly used, but recently, photoinitiators were used as alternatives that allowed conducting the polymerization under ITP conditions.[269]

The first example of photoinitiated ITP was a degenerative iodine transfer process under visible light irradiation using dimanganese decacarbonyl $\left(\mathrm{Mn}_{2}(\mathrm{CO})_{10}\right)$ in conjunction with alkyl iodide compounds.[270-272] The photochemical homolysis of the metal-metal bond of $\mathrm{Mn}_{2}(\mathrm{CO})_{10}$ generated a highly reactive metal-centered radical $\bullet \mathrm{Mn}(\mathrm{CO})_{5}$, which directly abstracted iodine atoms from alkyl iodides. The newly formed carbon-centered radicals not only initiated ITP of unconjugated monomers such as vinyl acetate and vinylidene fluorides, but also conjugated monomers such as styrenes and acrylates in the presence of appropriate alkyl iodide-based initiators. The generated $\mathrm{I}-\mathrm{Mn}$ bond in $\mathrm{I}-\mathrm{Mn}(\mathrm{CO})_{5}$ was too stable to reversibly deactivate propagating radicals and the system mainly operated by a degenerative chain transfer mechanism (

Figure 32).

\section{Insert Figure 32.}

The system provided well-defined polymers with narrow molecular weight distribution and the experimental molecular weights relatively close to theoretical values and temporal control for the growth of polymer chains by switching on/off the light source. This system was also applied to the preparation of well-defined poly(vinylidene fluoride) and its copolymers using a variety of alkyl and perfluoroalkyl halides (Cl, Br, I).[273] The visible light-induced ITP using bifunctional alkyl and perfluoroalkyl iodides afforded telechelic poly(vinylidene fluoride)s with very high (> $95 \%$ ) iodide chain-end fidelity, which were further utilized for preparation of a series of ABA triblock copolymers containing vinyl acetate, tert-butyl acrylate, methyl methacrylate, isoprene, styrene, and acrylonitrile segments.[274-276] 
The influence of UV light on photomediated ITP was also investigated under various experimental conditions.[277] ITPs of butyl methacrylate with cyanopropyl iodide as chain transfer agent and a conventional initiator, AIBN, were conducted at $60{ }^{\circ} \mathrm{C}$ under dark conditions and in the presence of UV light. The UV irradiation not only provided a significant increase in the molar-mass of the polymers but also lowered molecular weight distribution from above 1.5 down to 1.1 compared to non-irradiated system. The $\mathrm{C}-\mathrm{I}$ bond was light sensitive and homolytically cleaved to give R• and I• radicals upon UV irradiation. Since the light produces radicals needed for the ITP, its use not only accelerated the polymerization rate but also allowed formation of polymers with narrow molecular weight distributions. The effect of UV light on the polymerization rates was also investigated by continuous and intermittent UV irradiation experiments. The system was perfectly dormant with no monomer conversion occurring during the dark time, whereas the chain growth could be immediately restarted upon further irradiation.

Recently, a new concept for ITP was developed and consists of an alkyl iodide (dormant species) and an amine (catalyst) and uses visible light (350-600 nm).[278] It is among the most simple, inexpensive, and robust photoinduced LRPs. Mechanistically, the key reaction is the photolysis of free dormant species and dormant species/catalyst complex, which frequently occurs as the main activation process. The subsequent photochemical dissociation of this complex prevented "irreversible" reactions in the process and enabled the polymerization of a variety of acrylates to yield relatively well-defined polymers $\left(M_{\mathrm{w}} / M_{\mathrm{n}}<1.4\right)$. The temporal control of the system was also demonstrated by switching light "on" and "off". No polymerization occurred in the dark, meaning that the formed amine complex efficiently quenched the propagating radicals. By turning on the light, the restoration of the polymer chain growth of MMA was achieved in all cycles. The wavelength flexibility of this process further was extended to the visible range by employing various organic dyes $\left(\mathrm{CY}-1\left(\lambda_{\max }=530 \mathrm{~nm}\right)\right.$ and CY-3 $\left(\lambda_{\max }=602 \mathrm{~nm}\right)$ cyanine dyes, and SP-1 $\left(\lambda_{\max }=718 \mathrm{~nm}\right)$ spiropyran dye $)$ as photocatalysts.[279] Although, the similar mechanism was described, the photocatalyst acted as antenna that transferred light energy to cleave the $\mathrm{C}-\mathrm{I}$ bond of the complex (

Figure 33). This process was responsive to the irradiation wavelength; the polymerization could be conducted at the desired wavelengths by choosing suitable organic dyes. All three organic dyes produced well-defined polymers $\left(M_{\mathrm{w}} / M_{\mathrm{n}}<1.3\right)$ in good yields. The process was compatible with various functional groups and could be used to synthesize a wide range mechanistically different block copolymers in a one-pot mode. A block copolymer of MMA and Valero lactone (VL) was prepared by selectively regulating ITP and ring-opening polymerization using 
orthogonal irradiation techniques. The visible light exposure of the reaction mixture led to PMMA with 67\% yield, while no conversion of VL could be observed, consistent with selective light irradiation. The following UV light irradiation $(\lambda=350-380 \mathrm{~nm})$ resulted to $80 \% \mathrm{VL}$ monomer conversion with only $1 \%$ further MMA conversion, and resulted in formation of a welldefined block copolymer $\left(M_{\mathrm{w}} / M_{\mathrm{n}}=1.2\right)$. By taking advantage of the wavelength-responsive nature of this technique it might lead to one-pot synthesis of block copolymers using different polymerization mechanisms.

\section{Insert Figure 33.}

\subsection{Photomediated TERP}

Photochemical stimuli was efficiently implemented for the activation of organotelluriummediated radical polymerization (TERP) of meth(acrylate)s.[280] Two distinct activation mechanisms, namely the degenerative chain transfer and reversible termination, were involved and the TERP process mainly occurred by the degenerative chain transfer mechanism (

Figure 34).[281] The direct photolysis of the organotellurium compound led to homolytic cleavage of $\mathrm{C}-\mathrm{Te}$ bond with high quantum yield, 0.84 . The directly generated tertiary carboncentered radicals were responsible for the quantitative initiation of TERP even under visible light. The reversible deactivation process not only prevented irreversible termination reactions but also provided the synthesis of well-defined polymers with narrow molecular weight distribution $\left(M_{\mathrm{w}} / M_{\mathrm{n}}=<1.2\right)$, with the exception of poly(acrylic acid) which was slightly broader, $M_{\mathrm{w}} / M_{\mathrm{n}}=$ 1.34 .

\section{Insert Figure 34.}

In order to extend its wavelength sensitivity into the visible light range, ditelluride compounds, having a stronger absorption coefficient, were used the TERP process.[282] Since the quantum efficiency of organotellurium compounds was so high, the light intensity of the TERP process could be reduced by either using filters or altering UV light source with visible light sources like LED lamps. Indeed by tuning of the light intensity, well-defined polymers with narrow molecular weight distributions were synthesized under LED irradiation with or without a neutral density filter. In addition, no polymerization occurred in the absence of light, indicating temporal control of the polymer chain growth between active and inactive states by turning the light source on and 
off. However, the organotellurium compounds were very sensitive to air and light, foul-smelling, and difficult to handle. Because of these drawbacks, the development of simple preparation methods for organotellurium compounds still remains a challenging topic. In a recent study, a highly reactive benzoyl phenyltelluride, easily prepared from diphenyl ditelluride and benzoyl chloride in one-step, was developed for the photomediated TERP of various monomers under mild condition, visible light irradiation $(400-500 \mathrm{~nm})$ at room temperature.[283] Although the process was not feasible for styrenes and methacrylates, it produced well-defined polymers with narrow molecular weight distributions as low as 1.2 to 1.3 from acrylamides and acrylates.

\section{Radical intermediates in other photomediated controlled polymerizations}

As discussed in previous sections, photomediated reactions generate free radicals which promote controlled radical polymerization. However in addition to free radicals, other reactive intermediates such as radical anions and radical cations, derived from photoredox catalyzed reactions, could participate in several other controlled polymerizations.

A photoinduced ring-opening metathesis polymerization (ROMP) was developed and mediated by pyrylium salts acting as metal-free photoredox catalysts.[284] Pyrylium salts have been identified as efficient catalysts for photoinduced redox reaction in organic transformations.[285, 286] The polymerization of norbornene (

Figure 35), with a relatively high ring strain among ROMP monomers, was carried out with 1ethoxy-1-propene as initiator and a pyrylium salt in a molar ratio of 1000:1:0.03 in dichloromethane under a blue LED irradiation $(450$ - $480 \mathrm{~nm})$. After $120 \mathrm{~min}$ of irradiation, conversion of norbornene reached $61 \%$ based on ${ }^{1} \mathrm{H}$ NMR, and polynorbornene (PNB) was formed with $M_{\mathrm{n}}=60,200$ and $M_{\mathrm{w}} / M_{\mathrm{n}}=1.6$, which was close to the theoretical value of 57,400 . The synthesized PNB was confirmed by ${ }^{1} \mathrm{H}$ NMR analysis. $M_{\mathrm{n}}$ increased with conversion, and the reaction could be stopped or restarted by turning the irradiation source off or on.

\section{Insert Figure 35 .}

The "living" property and "on/off" behavior of this photoinduced metal-free ROMP was clarified by the proposed mechanism (Error! Reference source not found.). Vinyl ethers with oxidation potentials in the range of 1.43 to $1.30 \mathrm{~V}$ vs SCE, could be feasibly oxidized by the excited state of pyrylium cations shown in 
Figure 35. The oxidation potential was calculated to be $1.74 \mathrm{~V}$ vs SCE.[287] After the electron transfer from vinyl ether A to the excited pyrylium cation, a vinyl ether radical cation $\mathbf{B}$ was formed as a key intermediate, which subsequently reacted with a cycloalkene monomer to provide the $[2+2]$ cycloaddition intermediate C. Due to high ring strain, ring-opening of cyclobutane radical cation $\mathbf{C}$ formed $\mathbf{D}$ with radical cation chain end, which continually propagated with additional monomers to provide polymer chain $\mathbf{E}$. It was also proposed that the propagating radical cation had a dynamic redox couple with the reduced pyrylium species, which allowed the reduction of radical cation chain end to terminate polymerization $(\mathbf{E} \rightarrow \mathbf{F})$. This oxidation and reduction cycles represented the activation and deactivation cycles in a controlled polymerization.

\section{Insert Scheme 13.}

After this original methodology for the synthesis of PNB, photoinduced metal-free ROMP of dicyclopentadiene (DCPD), another common ROMP monomer, was carried out to form linear polyDCPD.[288] The cyclopentane moiety in polyDCPD also allowed the preparation of a crosslinked polyDCPD by thiol-ene chemistry.

\section{Combination of photomediated radical polymerizations and click processes}

The term "click chemistry" defines a collection of reactions allowing for the efficient building of chemical libraries under mild reaction conditions using readily available reagents. [289] The criteria for being classified as click chemistry reaction are wide in scope, modular, proceeds in high yields, with none, or inoffensive by-products, tolerant to many functional groups and final products that can be easily isolable by non-chromatographic methods. The copper(I)-catalyzed azide-alkyne cycloaddition (CuAAC) was the first example of "click" chemistry.[290, 291] Since ATRP and CuAAC processes are both based on a similar $\mathrm{Cu}(\mathrm{I})$ catalytic system, their sequential or simultaneous combinations provide versatile routes for the construction of a variety of complex and functional macromolecular structures from heterofunctional molecules.[292-295] The mechanistic combination of ATRP and CuAAC techniques were usually achieved in a sequential manner.[296-302] The general approach was either the use of an alkyne-functionalized ATRP initiator to synthesize a polymer bearing an $\alpha$-alkynyl end-group or a nucleophilic displacement of the terminal halogen of polymers prepared by ATRP with sodium azide. Application of a subsequent CuAAC click reaction with these reactive polymers afforded the desired product in a quantitative yield under ambient conditions. On the other hand, there were a 
few examples of the simultaneous combination of ATRP and CuAAC techniques.[299, 300, 303305] The combination of photoinduced CuAAC and ATRP was also accomplished and the results comparable with the thermally combined version. Quite recently, synthesis of block[306] and graft[307] polymers were successfully achieved by combined one-pot photoinduced ATRP and CuAAC protocols (

Figure 36). For preparation of the block copolymer, clickable azido-terminated polystyrene and prop-2-ynyl 2-bromo-2-methylpropanoate as ATRP initiator were mixed with methyl methacrylate and $\mathrm{Cu}(\mathrm{II}) / \mathrm{PMDETA}$ in acetonitrile. Photochemically regenerated $\mathrm{Cu}(\mathrm{I})$ activators could activate both CuAAC and ATRP processes under UV light irradiation. After $24 \mathrm{~h}$, a polystyrene- $b$-poly(methyl methacrylate) block copolymer was obtained with $68 \%$ monomer conversion. The monomodal GPC peak with a clear shift to a higher molecular weight region confirmed the successful block copolymer formation without any detectable contaminant homopolymer.

\section{Insert Figure 36.}

The utility of this one-pot protocol for the synthesis of well-defined polystyrene- $g$-poly(methyl methacrylate) was also demonstrated.[307] In this case, a side-chain azide functional copolymer, namely poly(styrene-co-4-azidomethylstyrene), was used instead of azido-terminated polystyrene. After $4 \mathrm{~h}$ of UV light irradiation, the graft copolymer was obtained with good conversion of added monomer (72\%) under mild reaction conditions. The reaction was carried out at room temperature without the necessity of an added photosensitive molecule. By varying the recipe and reaction conditions, choosing the proper ligand, initiator, and solvent, the combined one-pot photoinduced ATRP and CuAAC enabled the construction of the desired macromolecular structures in a well-controlled manner. ATRP was successfully combined with ring-opening, ionic and even polycondensation processes and this approach can be extended to photomediated

processes. [308-311] In a similar approach, PET-RAFT was combined with orthogonal ROMP to synthesize block copolymer at ambient temperature.[312] Very recently, a photoacid-mediated ring opening polymerization irradiated by visible light was combined with photoRAFT to result in a one-pot sequential polymerization under two different wavelength. [313] 


\section{Surface-initiated photomediated radical polymerization}

The temporospatial control of photoinduced reactions is ideal for manipulation of surfaces. Photopolymerizations in specific areas can be used to directly grow polymer brushes in specific patterns following CRP protocols (hence retention of end group functionality and the ability to form block copolymers). In the following, the performance of photomediated RDRP reactions for surface modification and patterning is highlighted.

\subsection{PhotoATRP}

The first reported copper-mediated photoATRP surface-initiated polymerization used $0.5 \mathrm{~mol} \%$ $\mathrm{CuCl}_{2}$ /bpy as catalyst and $\mathrm{TiO}_{2}$ as photosensitizer.[60] Various hydrophilic, or even ionic, methacrylates such as MeOGEMA, NIPAAm and 3-sulfopropyl methacrylate (SPMA) were grafted from a flat gold substrate or titanium nanowires with water-methanol mixtures as solvent at low light intensities. SPMA films grew linearly with time and reached more than $200 \mathrm{~nm}$ in thickness (

Figure 37). Increased thicknesses were found with increased light intensities, whereas the $\mathrm{TiO}_{2}$ concentration have no influence once a certain level was exceeded.

\section{Insert Figure 37.}

Polymer brush patterns were generated via combination of $\mu \mathrm{CP}$ and photoATRP.[60] More recently, $\mathrm{TiO}_{2}$ particles were imprinted with a dye, yielding faster reactions and making the reaction accessible to the use of visible light $\left(66 \mathrm{~mW} / \mathrm{cm}^{2}\right)$.[55] Remarkable nanoscale-resolved patterns were generated with anodized aluminum oxide as a photomask. Recently, the grafting from a nanocomposite of $\mathrm{TiO}_{2}$ and reduced graphene oxide as photosensitizer were reported in combination with tetrasulfonated copper phthalocyanine. The resulting pMMA brushes were cleaved in a saponification reaction over 7 days and showed a dispersity of around 1.3 and $M_{n}$ $=25,000 .[58]$

The first copper-photoATRP grafting without photosensitizer was described for MMA from silicon substrates.[80] PMMA films of $15 \mathrm{~nm}$ thickness were formed after 5 hours irradiation. Successful block copolymer formation with tBMA and micropatterning indicated the controlled nature of the initial grafting from polymerization and exemplified the full potential of photoATRP grafting. Photosensitizer-free copper-photoATRP of acrylates was first reported to 
graft methyl acrylate from cellulose substrates.[85] Subsequent SEC characterization of the cleaved brushes confirmed the livingness of the procedure, however the polymers displayed higher dispersities and higher molecular weights than polymers formed in the presence of a sacrificial initiator. Interestingly, block copolymer formation from secondary chlorinated initiators was feasible, although never reported for polymerizations in solution.

PhotoATRP grafting of various acrylates from silicon substrates was investigated.[76] $\mathrm{N}-2$ hydroxypropylmethacrylate and MeOEGMA were grafted from substrates under photoATRP conditions.[82] In both cases, (meth)acrylate monomers, $\mathrm{CuBr}_{2} / \mathrm{Me}_{6} \mathrm{TREN}$ (1/6) in DMSO were exposed to UV light (360 nm, $36 \mathrm{~W}$ ) with air cooling. [76, 82] The grafting rate was proportional to the irradiation time, even in the presence of ppb-range concentrations of catalyst. The catalyst concentration had some influence on the polymerization rate, and both very low (100 ppb) and relative high (100 ppm) copper catalyst concentrations led to a decrease in final film thickness compared to medium concentrations (1-10 ppm),

Figure 38. The grafting rate was proportional to the monomer concentration. As reported for photoATRP in solution, the choice of solvent had significant influence on the polymerization rate. Interestingly, monomers with high excluded volume interactions such as $t$-butyl, 2-ethylhexyl acrylate or the ionic [2-acryloyloxy)ethyl]trimethylammonium chloride led to film thicknesses of to up to $950 \mathrm{~nm}$ after 1 hour, whereas film thickness with other monomers stayed below $250 \mathrm{~nm}$. Reinitiation and block copolymer formation of $t$-butyl and 2-hydroxyethyl acrylate were performed on the polymer brushes. No phase separation was found for the PtBA and the poly(2hydroxyethyl acrylate) blocks, rather a gradual composition shift from a 2-hydroxyethyl acrylate enriched upper layer towards almost pure $t \mathrm{BA}$ at the polymer-silicon interface was revealed via X-ray photoelectron spectroscopy (XPS) depth profiling (

Figure 38).[76] A similar result was reported for $N$-(2-hydroxypropyl)methacrylamide and MeOEGMA.[82] ToF-secondary ion mass spectrometry (SIMS) depth profile showed maximum of the bromine concentration at the polymer/air interface and the polymer/silicon interface depending on the choice of sputter ion.[76] Basic spatial control was achieved via a shadow mask, but temporal control remained challenging.[76, 82] Antifouling properties of poly(HMPA) brushes were also demonstrated. [82] Photochemical modification of polypropylene surface with benzophenone containing bromoisobutyrate as ATRP initiator was followed by ATRP of dimethylaminoethyl methacrylate and subsequent quaternization with ethyl bromide. The 
quaternary ammonium moieties showed antibacterial activity against Escherichia coli (E. coli). The biocidal activity of the resultant surfaces depends on the amount of the grafted polymers (the number of available quaternary ammonium units). With the same grafting density, the surface grafted with relatively high MW polymers $\left(M_{\mathrm{n}}>10,000\right)$ showed almost $100 \%$ killing efficiency (killing all of the input E. coli $\left(2.9 \times 10^{5}\right)$ in the shaking test), whereas a lower biocidal activity (85\%) was observed for the surface grafted with shorter PQA chains $\left(M_{\mathrm{n}}=1,500\right)$.[314] In a related approach, thermoresponsive star polymers were photo-crosslinked in order to control of cell-surface interactions to enhance cell detachment.[315]

\section{Insert Figure 38 .}

Besides $\mathrm{Cu}$ - and Ir-based catalysts, Fe[99] and Mn-mediated[316] photoATRP grafting protocols were also reported. In the Mn-mediated photoATRP processes $\operatorname{Mn}_{2}(\mathrm{CO})_{10}$ was used to graft NIPAAm, styrene, $N$-vinylpyrrolidone and $t$-butyl methacrylate from various substrates.[316] A linear increase up to $160 \mathrm{~nm}$ film thickness within only 6 min was observed. Despite these thick films, XPS measurements showed intense signals originating from the substrate, indicating a less than homogenous grafting. However, block copolymer formation, reinitiation and other control experiments were not assessed. Iron-catalyzed photoATRP grafting of pentafluoropropyl acrylate was reported.[99] Photomasks were employed to generate spatially resolved patterns. Reinitiation and block copolymer formation were only demonstrated for the polymer film.

Ir-mediated photoATRP of MMA from silicon substrates carrying a self-assembled monolayer (SAM) of BiB-based was successful, Figure 39.[317]. The substrates were covered with a thin glass slide to ensure short path lengths of the employed visible light. MMA, OEGMA and various fluorinated methacrylates were polymerized and yielded film thicknesses between 60 and $120 \mathrm{~nm}$ within a one hour brush polymerization time.[317, 318] Ionic monomers such as 2methacryloyloxyethyl phosphorylcholine and sulfobetaine methacrylate formed only very thin layers, even with trifluoroethanol as co-solvent.[318] Catalyst concentrations were kept at $50 \mathrm{ppm}$ for MMA,[317] but were increased to 500 ppm for OEGMA and fluorinated monomers.[318] The linear relationship between irradiation time, light intensity and film thickness and performed

chain extension reactions confirmed the controlled nature of the reported grafting from procedures.[317, 318] This gave a direct access to 3D architectures on the surface via use of surface of photomasks with areas of different optical densities.[317] Due to short diffusion path lengths of the excited Ir-catalyst species, patterns with $\mu \mathrm{m}$ resolution were readily accessible. On- 
off experiments confirmed good temporal control. In a recent report, grafting on commercial PVC with Ir based catalyst was successful in solution and on surfaces. [319]

\section{Insert Figure 39.}

Block copolymers of OEGMA and TFEMA were grafted from surfaces, while the high hydrophobicity of TFEMA and its pronounced surface activity reduced its chain extension capability especially for thick layers of pTFEMA.[318] $\mathrm{CF}_{3}$ moieties were present at the polymer/air interface even when pOEGMA was grafted as the second, outer block, underlining the low surface energy of $\mathrm{CF}_{3}$. The sequential use of Ir-mediated photoATRP with thermal $\mathrm{Cu}-$ mediated ATRP gave access also to ionic monomers. The generated micron-scale patterns were investigated with optical microscopy, scanning transmission X-ray microscopy (STXM) and SIMS. Ir-mediated photoATRP was also reported for the modification of membranes with antifouling polymers such as hydroxyethyl methacrylate (HEMA), GMA and OEGMA.[320] Reaction times were extended to 24 hours under fluorescent light (50W) and a linear relationship of grafting yield (mass) and time was up to $12 \mathrm{~h}$ exposure time, irrespective of the catalyst concentration which was generally kept below 300 ppm. Furthermore, a linear dependency on the monomer concentration was reported by the same group.

Recently, a visible-light mediated phenothiazine-catalyzed metal-free ATRP was extended to fabricate of well-defined polymer brushes from flat, curved surfaces and particle surfaces.[321] Single-layer patterns, gradient structures as well as block copolymer architectures could be readily obtained under irradiation from compact fluorescent lamps and natural sunlight. A linear relationship between brush height and irradiation time was observed, confirming the "living" nature of whole process. Moreover, chain-extension of a uniform PMMA brush ( 30 nm) with TFEMA increased the brush thickness of $26 \mathrm{~nm}$, indicating a good retention of active chain ends. In a following work, 2-bromo-2-phenylacetate based tetherable initiators were prepared and gave better initiation efficiency and performance in PTZ-catalyzed metal-free SI-ATRP. [322]

\subsection{Photoiniferter and photoRAFT}

The first photoiniferter grafting methodology was reported in 1996.[323] Various monomers such as MMA, styrene and dimethylaminopropyl acrylamide were grafted from polymer substrates armed with $30 \mathrm{~mol} \%$ diethyldithiocarbamate functionalities. These polymer substrates became more readily available after a dithiocarbamate-containing methacrylate monomer was synthesized.[324] Triblock copolymers were grafted in a controlled manner, visualized via fluorescence microscopy after staining, and film thicknesses up to $420 \mathrm{~nm}$ were reached.[323] 
Diblock copolymer brushes were also grafted from a silicon substrate, with a significantly reduced grafting rate, potentially due to the lower amount of initiating sites.[325] Photomask and neutral-density filters were employed to generate complex 3D structures,

Figure 40.[323, 326] More elaborated patterns with polymer grafting including formation of a gradient in film thicknesses were generated with $x$-y-adjustable photomasks.[327] A methodology for the generation of 2D and 3D patterned polymer substrates was developed.[328]

Patterns of second[329] or even third[330] generation hyperbranched brushes on glass slides were prepared from poly(p-chloromethylstyrene) brushes functionalized with additional iniferter initiators (

Figure 40). Hydrophilic monomers such as MAA, OEGMA or DMAAm were grafted from the new iniferter initiator sites in a subsequent polymerization step.[329] The growth of PMMA brushes was monitored in situ via quartz crystal microbalance (QCM) and provided a film thickness of $290 \mathrm{~nm}$ PMMA which corresponded with a molecular mass of 137,500.[331] In addition to the functionalization of flat surfaces, the surfaces of nanoparticles of alumina and silica were also successfully modified via photoiniferter, underlining the high versatility of photoiniferter procedures for any surface modification.[332-334]

\section{Insert Figure 40.}

The investigation of the kinetics of photoiniferter surface grafting showed a linear evolution of film thickness with time only for short reaction times, followed by a decrease in polymerization rate indicating the presence of termination events.[335] This behavior is expected for any surfaceinitiated RDRP.[336] The addition of a diethyldithiocarbamate functionality was suggested to suppress chain transfer events and to improve the polymerization control.[334, 337, 338] The effect of diethyldithiocarbamates on the grafting kinetics was investigated[339] and the reinitiation ability dependence of the concentration of the diethyldithiocarbamate tetraethylthiuram disulfide was modeled in a subsequent study.[340] Furthermore, it was suggested that an increase the concentration of deactivating species could improve the livingness.[335] Also the influence of the light intensity was investigated. Similar to photoATRP, 
a linear dependence of the grafting rate on the monomer concentration was identified. A methodology was developed to generate patterns with gradient film thicknesses from silicon substrates.[341]

Temperature- and $\mathrm{pH}$-responsive polystyrene particles were prepared using a photoiniferter to graft pNIPAAm and $\mathrm{p}$ (NIPAAm-co-acrylic acid) and pNIPAAm- $b$-p(NIPAAm-co-acrylic acid) as well as p(NIPAAm-co-acrylic acid)- $b$-pNIPAAm diblock copolymers, respectively, from polystyrene particles featuring DC-initiators sites.[342, 343] Temperature, $\mathrm{pH}$ and ionic strengthresponsive diblock copolymer brushes of PMAA and PNIPAAm were grafted from silicon substrates.[344] The ellipsometric film thickness of the diblock copolymer brushes on silicon substrate was expanded by factor up to 4 via changes in $\mathrm{pH}$ and temperature or addition of ions with respect to the dry film thicknesses. Surface-bound PNIPAAm blocks show LCST behavior over a temperature range of around $10{ }^{\circ} \mathrm{C}$, in contrast to a sharp transition in solution. When a disulfide-containing photoiniferter initiator was employed to form a SAM and subsequently graft pNIPAAm from gold substrates, a significantly wider temperature range was not observed for LCST behavior.[345] In a further study, the topographic changes upon $\mathrm{pH}$ changes were characterized via atomic-force microscopy (AFM).[346] Very recently, conductive polymer brushes based on polythiophene were reported.[347] 3-Methylthienyl methacrylate was grafted via a photoiniferter mechanism and was then subsequently oxidized and doped with iodine to give a conductive polythiophene polymer parallel to the polymethacrylate backbone (

Figure 41). Film thicknesses up to $290 \mathrm{~nm}$ were reported, and the conductivity decreased by factor 7 within one hour after doping, indicating oxidation.

\section{Insert Figure 41.}

Hydrophilic,[348, 349] ionic,[350] and carboxybetaine[351-355] monomers were employed for the preparation of antifouling coatings on gold, polymers and silicon substrates via photoiniferter grafting from reactions. Furthermore, monomers based on amino acids were also reported in photoiniferter grafting polymerization.[356, 357] Protein immobilization via post-polymerization EDC coupling and controlled drug release systems based on drugs encapsulated in block copolymer brushes were reported.[348] In one example bovine albumin protein was attached to a dithiocarbamate-initiator on glass slides.[358] Subsequently poly(dimethylacrylamide) and polyNIPAAm brushes were grown from the surface. Brushes featuring bovine albumin reached heights of 400 to $500 \mathrm{~nm}$. The combination of poly(carboxybetaine) brushes with long brushes 
for specific protein antibody binding enabled access to antifouling films with high loading binding characteristics.[353-355] A similar approach based on polyPEGMA was reported.[349]

Another example of selective particle functionalization was synthesis of $\mathrm{SiO}_{2}$ particles functionalized with multiple layers of molecular imprinted polymers, which were subsequently successfully employed for the separation of D- and L-phenylalanine anilide in affinity column chromatography.[359] Recently, MIPs for the anti-inflammatory drug fenbufen were generated using a photoiniferter, leading to potential applications in analytical devices.[360] Likewise, hybrid-MIPs for the identification and subsequent oxidation of catechol were produced using photoiniferter.[361] Unrelated to that, photoiniferters were also employed to produce microfluidic devices, underpinning again the versatility of the photoiniferter methodology.[362]

Recently, initiator-free photoRAFT was employed to graft PMA brushes from crosslinked pEGDA particles.[208] An initiation efficiency of 95\% was determined for the embedded trithiocarbonate RAFT agent. No additional photoinitiator was used since radicals were directly generated from the transfer agent 2-(((butyltio)carbonothiolyl)thio)propanoic acid upon exposure to blue LED-light. PhotoRAFT was employed to graft PAA[363, 364] and PHEMA[365] from polypropylene microporous membranes, although only a few repeating units were attached in the presence of chain transfer agent in solution. Subsequently, a PNIPAAm block was grafted via thermal RAFT to yield block copolymers.[363, 365] Further control experiments were not performed. These modifications of the surface of membrane were conducted by initially exposing the surface to high power UV light in the presence of benzophenone which subsequently formed surface-bound semipinacol radicals to initiate the polymerization in the presence of a chain transfer agent.

The potential of photoRAFT for the production of $\mathrm{MIP}-\mathrm{SiO}_{2}$ composite beads was demonstrated.[366] Photoiniferter and photoRAFT, activated with a surface-bound azoinitiator in the presence of a RAFT agent, were compared. PhotoRAFT and photoiniferter surface functionalization were carried out at $15{ }^{\circ} \mathrm{C}$. L-Phenylalanine anilide was imprinted on a poly(MAA-co-EGMA) matrix. A loss of sulfur concentration was observed for both systems, indicating a loss of control potentially due to recombination events. However, the same result was found in an earlier study where photoiniferter was reported to allow successful formation of various layers.[359] In contrast to a photoiniferter procedure, the photoRAFT system maintained the pore size distribution of the original silica particles.[366] Furthermore, particles modified with the photoRAFT featured a more homogenous polymer film. With respect to enantioselectivity 
and retention in column chromatography, photoRAFT surface functionalization was superior to photoiniferter grafting. Silica particles functionalized via photoiniferter grafting were not able to separate racemic mixtures of phenylalanine anilide, whereas the respective particles, after photoRAFT grafting, featured good separation for film thicknesses beyond $1 \mathrm{~nm}$, which was attributed to formation of more homogenous films. Retention factor and enantioselectivity were linearly dependent on the graft density for photoRAFT, in contrast to the results of photoiniferter grafting from reactions where a bell shaped curve was observed.

\subsection{Photomediated NMP}

The development of novel photosensitive alkoxyamines extended the use of NMP process in many applications such as photolithography, microfluidic, data storage devices, 3D-printing, biochips, etc. For example, a homogeneous acrylate-based multilayered film with a thickness close to $50 \mathrm{~nm}$ and a good spatial resolution could be prepared by using photosensitive alkoxyamines.[367] The living character of the photoinduced NMP enabled reinitiation of the polymerization producing multi-layered objects with nanometric scale thickness. The photoinduced NMP process was also proposed and developed for fabrication functional micropatterns with complex structures such as hydrophilic/hydrophobic or luminescent surfaces simply by using a mask (

Figure 42). [368] The self-healing process was reported in covalent polymer networks via the photodissociation of alkoxyamine junctions.[369]

\section{Insert Figure 42.}

\subsection{PhotoCRP in direct laser writing}

Direct laser writing (DLW) with photoinduced controlled radical polymerizations was first reported for photomediated NMP.[367] Line widths of $1.5 \mu \mathrm{m}$ were achieved with a $375 \mathrm{~nm}$ laser $(16 \mathrm{~mW})$ and an inverted microscope objective with piezoelectronically positioned sample. Polymeric microstructures of up to $2 \mu \mathrm{m}$ height were grafted from the surface of a crosslinked polymer film featuring suitable alkoxyamine groups with only $30 \mathrm{~ms}$ irradiation time. The structure height was proportional to the irradiation time for the first $10 \mathrm{~ms}$. Recently, microstructures grafting via direct laser writing on Ir-mediated photoATRP was reported ( 
Figure 43).[126] An almost linear dependence of the structure height on the power of the laser employed Nd:Yag microlaser (532 nm) was reported. In contrast to photomediated NMP, Irmediated photoATRP required significantly higher laser powers and structure heights were limited to less than $350 \mathrm{~nm}$.

\section{Insert Figure 43.}

\section{Summary and perspective}

Photomediated controlled radical polymerizations and other processes are versatile methods that allow synthesis of well-defined polymers with predesigned structures. Additionally, the photomediated conditions allow creating specialized materials with interesting features. The photomediated processes can be conducted at ambient or subambient temperatures. Temporal and spatial controls over the reaction could be easily controlled by switching on/off the irradiation source. Fine tuning of radical formation by controlling light intensity and wavelength leads to precisely controlled individual macromolecules and hybrid polymeric structures due to the exact control of radical concentration. In addition to these advantages of photomediated processes, the use of photochemistry also overcame challenges inherent in thermally mediated reactions. The excited molecules, which absorb the energy of photons, are active catalysts but they could not be generated under thermal conditions. Photoredox-mediated and metal-free ATRP could only be accessed under photochemical conditions.

While significant progress has been achieved, future research should target more environmentally friendly and refined systems. For instance, development of catalyst and processes using milder irradiation and longer wavelength sources in the presence of photosensitizers is attractive, since most systems described in this review required high energy irradiation sources $(350-500 \mathrm{~nm})$. The design of novel catalysts containing more than one distinct catalytic center could provide a selective reactivity under different irradiation conditions. Furthermore, the different reactivity of more sophisticated catalysts might possibly target longer wavelengths and lower light intensity.

Acknowledgments. KM and XP acknowledge support from NSF (CHE 1400052). TJ and JL are grateful for support from the Flemish Fonds Wetenschappelijk Onderzoek (FWO). 


\section{References}

[1] Matyjaszewski K, Davis TP, editors. Handbook of Radical Polymerization. Hoboken: Wiley-Interscience, 2002.936 pp.

[2] Braunecker WA, Matyjaszewski K. Controlled/living radical polymerization: Features, developments, and perspectives. Prog Polym Sci 2007;32:93-146.

[3] Goto A, Fukuda T. Kinetics of living radical polymerization. Prog Polym Sci 2004;29:329-385.

[4] Zhong M, Matyjaszewski K. How fast can a CRP be conducted with preserved chain end functionality? Macromolecules 2011;44:2668-2677.

[5] Nicolas J, Guillaneuf Y, Lefay C, Bertin D, Gigmes D, Charleux B. Nitroxide-mediated polymerization. Prog Polym Sci 2013;38:63-235.

[6] Allan LEN, Perry MR, Shaver MP. Organometallic mediated radical polymerization. Prog Polym Sci 2012;37:127-156.

[7] Wayland BB, Poszmik G, Mukerjee SL, Fryd M. Living Radical Polymerization of Acrylates by Organocobalt Porphyrin Complexes. J Am Chem Soc 1994;116:7943-7944.

[8] Poli R. Relationship between one-electron transition-metal reactivity and radical polymerization processes. Angew Chem Int Ed 2006;45:5058-5070.

[9] Kamigaito M, Ando T, Sawamoto M. Metal-Catalyzed Living Radical Polymerization. Chem Rev 2001;101:3689-3745.

[10] Matyjaszewski K, Xia J. Atom Transfer Radical Polymerization. Chem Rev 2001;101:2921-2990.

[11] Wang JS, Matyjaszewski K. Controlled/"living" radical polymerization. atom transfer radical polymerization in the presence of transition-metal complexes. J Am Chem Soc 1995;117:5614-5615.

[12] Matyjaszewski K. Atom Transfer Radical Polymerization (ATRP): Current Status and Future Perspectives. Macromolecules 2012;45:4015-4039.

[13] Fischer H. The Persistent Radical Effect: A Principle for Selective Radical Reactions and Living Radical Polymerizations. Chem Rev 2001;101:3581-3610.

[14] Jakubowski W, Matyjaszewski K. Activators Regenerated by Electron Transfer for Atom-Transfer Radical Polymerization of (Meth)acrylates and Related Block Copolymers. Angew Chem Int Ed 2006;45:4482-4486. 
[15] Matyjaszewski K, Jakubowski W, Min K, Tang W, Huang JY, Braunecker WA, Tsarevsky NV. Diminishing catalyst concentration in atom transfer radical polymerization with reducing agents. Proc Natl Acad Sci USA 2006;103:15309-15314.

[16] Magenau AJD, Strandwitz NC, Gennaro A, Matyjaszewski K. Electrochemically Mediated Atom Transfer Radical Polymerization. Science 2011;332:81-84.

[17] Abreu CMR, Mendonça PV, Serra AnC, Popov AV, Matyjaszewski K, Guliashvili T, Coelho JFJ. Inorganic Sulfites: Efficient Reducing Agents and Supplemental Activators for Atom Transfer Radical Polymerization. ACS Macro Lett 2012;1:1308-1311.

[18] Konkolewicz D, Wang Y, Zhong M, Krys P, Isse AA, Gennaro A, Matyjaszewski K. Reversible-Deactivation Radical Polymerization in the Presence of Metallic Copper. A Critical Assessment of the SARA ATRP and SET-LRP Mechanisms. Macromolecules 2013;46:8749-8772.

[19] Guliashvili T, Mendonça PV, Serra AC, Popov AV, Coelho JFJ. Copper-Mediated Controlled/“Living” Radical Polymerization in Polar Solvents: Insights into Some Relevant Mechanistic Aspects. Chem - Eur J 2012;18:4607-4612.

[20] Percec V, Guliashvili T, Ladislaw JS, Wistrand A, Stjerndahl A, Sienkowska MJ, Monteiro MJ, Sahoo S. Ultrafast synthesis of ultrahigh molar mass polymers by metalcatalyzed living radical polymerization of acrylates, methacrylates, and vinyl chloride mediated by SET at 25 degrees C. J Am Chem Soc 2006;128:14156-14165.

[21] Tasdelen MA, Uygun M, Yagci Y. Photoinduced controlled radical polymerization in methanol. Macromol Chem Phys 2010;211:2271-2275.

[22] Tasdelen MA, Uygun M, Yagci Y. Photoinduced controlled radical polymerization. Macromol Rapid Commun 2011;32:58-62.

[23] Konkolewicz D, Schroder K, Buback J, Bernhard S, Matyjaszewski K. Visible Light and Sunlight Photoinduced ATRP with ppm of Cu Catalyst. ACS Macro Lett 2012;1:12191223.

[24] Mosnáček J, Ilčíková M. Photochemically Mediated Atom Transfer Radical Polymerization of Methyl Methacrylate Using ppm Amounts of Catalyst. Macromolecules 2012;45:5859-5865.

[25] Anastasaki A, Nikolaou V, Zhang Q, Burns J, Samanta SR, Waldron C, Haddleton AJ, McHale R, Fox D, Percec V, Wilson P, Haddleton DM. Copper(II)/Tertiary Amine Synergy in Photoinduced Living Radical Polymerization: Accelerated Synthesis of $\omega$ - 
Functional and $\alpha, \omega$-Heterofunctional Poly(acrylates). J Am Chem Soc 2014;136:11411149.

[26] Treat NJ, Sprafke H, Kramer JW, Clark PG, Barton BE, Read de Alaniz J, Fors BP, Hawker CJ. Metal-Free Atom Transfer Radical Polymerization. J Am Chem Soc 2014;136:16096-16101.

[27] Pan X, Lamson M, Yan J, Matyjaszewski K. Photoinduced Metal-Free Atom Transfer Radical Polymerization of Acrylonitrile. ACS Macro Lett 2015;4:192-196.

[28] Goto A, Ohtsuki A, Ohfuji H, Tanishima M, Kaji H. Reversible Generation of a CarbonCentered Radical from Alkyl Iodide Using Organic Salts and Their Application as Organic Catalysts in Living Radical Polymerization. J Am Chem Soc 2013;135:1113111139.

[29] Yamago S. Precision Polymer Synthesis by Degenerative Transfer Controlled/Living Radical Polymerization Using Organotellurium, Organostibine, and Organobismuthine Chain-Transfer Agents. Chem Rev 2009;109:5051-5068.

[30] Moad G, Rizzardo E, Thang SH. Living Radical Polymerization by the RAFT Process. Aust J Chem 2005;58:379-410.

[31] Chiefari J, Chong YK, Ercole F, Krstina J, Jeffery J, Le TPT, Mayadunne RTA, Meijs GF, Moad CL, Moad G, Rizzardo E, Thang SH. Living Free-Radical Polymerization by Reversible Addition-Fragmentation Chain Transfer: The RAFT Process. Macromolecules 1998;31:5559-5562.

[32] Lin CY, Marque SRA, Matyjaszewski K, Coote ML. Linear-Free Energy Relationships for Modeling Structure-Reactivity Trends in Controlled Radical Polymerization. Macromolecules 2011;44:7568-7583.

[33] Chatgilialoglu C, Ferreri C, Matyjaszewski K. Radicals and Dormant Species in Biology and Polymer Chemistry. ChemPlusChem 2016;81:11-29.

[34] Yagci Y, Jockusch S, Turro NJ. Photoinitiated Polymerization: Advances, Challenges, and Opportunities. Macromolecules 2010;43:6245-6260.

[35] Dietlin C, Schweizer S, Xiao P, Zhang J, Morlet-Savary F, Graff B, Fouassier JP, Lalevee J. Photopolymerization upon LEDs: new photoinitiating systems and strategies. Polym Chem 2015;6:3895-3912.

[36] Xiao P, Zhang J, Dumur F, Tehfe MA, Morlet-Savary F, Graff B, Gigmes D, Fouassier JP, Lalevée J. Visible light sensitive photoinitiating systems: Recent progress in cationic 
and radical photopolymerization reactions under soft conditions. Prog Polym Sci 2015;41:32-66.

[37] Tasdelen MA, Yagci Y. Photochemical Methods for the Preparation of Complex Linear and Cross-linked Macromolecular Structures. Aust J Chem 2011;64:982-991.

[38] Gruber HF. Photoinitiators For Free-Radical Polymerization. Prog Polym Sci 1992;17:953-1044.

[39] Carlini C, Angiolini L, Caretti D, Corelli E. Recent advances on photosensitive polymers: Polymeric photoinitiators. Polym Adv Technol 1996;7:379-384.

[40] Shao J, Huang Y, Fan Q. Visible light initiating systems for photopolymerization: status, development and challenges. Polym Chem 2014;5:4195-4210.

[41] Prier CK, Rankic DA, MacMillan DWC. Visible Light Photoredox Catalysis with Transition Metal Complexes: Applications in Organic Synthesis. Chem Rev 2013;113:5322-5363.

[42] Yoon TP, Ischay MA, Du J. Visible light photocatalysis as a greener approach to photochemical synthesis. Nature Chem 2010;2:527-532.

[43] Cismesia MA, Yoon TP. Characterizing chain processes in visible light photoredox catalysis. Chem Sci 2015;6:5426-5434.

[44] Xi Y, Yi H, Lei A. Synthetic applications of photoredox catalysis with visible light. Org Biomol Chem 2013;11:2387-2403.

[45] Chen M, Zhong M, Johnson JA. Light-Controlled Radical Polymerization: Mechanisms, Methods, and Applications. Chem Rev 2016 DOI: 10.1021/acs.chemrev.5b00671

[46] Dadashi-Silab S, Doran S, Yagci Y. Photoinduced Electron Transfer Reactions for Macromolecular Syntheses. Chem Rev 2016 DOI: 10.1021/acs.chemrev.5b00586

[47] Corrigan N, Shanmugam S, Xu J, Boyer C. Photocatalysis in organic and polymer synthesis. Chem Soc Rev 2016 DOI: 10.1039/C6CS00185H

[48] Zivic N, Bouzrati-Zerelli M, Kermagoret A, Dumur F, Fouassier J-P, Gigmes D, Lalevée J. Photocatalysts in Polymerization Reactions. ChemCatChem 2016;8:1617-1631.

[49] Dadashi-Silab S, Tasdelen MA, Yagci Y. Photoinitiated Atom Transfer Radical Polymerization: Current Status and Future Perspectives. J Polym Sci Part A Polym Chem 2014;52:2878-2888.

[50] Tasdelen MA, Uygun M, Yagci Y. Studies on Photoinduced ATRP in the Presence of Photoinitiator. Macromol Chem Phys 2011;212:2036-2042. 
[51] Yagci Y, Tasdelen MA, Jockusch S. Reduction of $\mathrm{Cu}$ (II) by photochemically generated phosphonyl radicals to generate $\mathrm{Cu}(\mathrm{I})$ as catalyst for atom transfer radical polymerization and azide-alkyne cycloaddition click reactions. Polymer 2014;55:3468-3474.

[52] Tasdelen MA, Ciftci M, Yagci Y. Visible Light-Induced Atom Transfer Radical Polymerization. Macromol Chem Phys 2012;213:1391-1396.

[53] Ciftci M, Tasdelen MA, Li W, Matyjaszewski K, Yagci Y. Photoinitiated ATRP in Inverse Microemulsion. Macromolecules 2013;46:9537-9543.

[54] Taskin OS, Yilmaz G, Tasdelen MA, Yagci Y. Photoinduced reverse atom transfer radical polymerization of methyl methacrylate using camphorquinone/benzhydrol system. Polym Int 2014;63:902-907.

[55] Li B, Yu B, Zhou F. Spatial Control over Brush Growth through Sunlight-Induced Atom Transfer Radical Polymerization Using Dye-Sensitized TiO2 as a Photocatalyst.

Macromol Rapid Commun 2014;35:1287-1292.

[56] Dadashi-Silab S, Tasdelen MA, Kiskan B, Wang X, Antonietti M, Yagci Y. Photochemically Mediated Atom Transfer Radical Polymerization Using Polymeric Semiconductor Mesoporous Graphitic Carbon Nitride. Macromol Chem Phys 2014;215:675-681.

[57] Dadashi-Silab S, Tasdelen MA, Asiri AM, Khan SB, Yagci Y. Photoinduced Atom Transfer Radical Polymerization Using Semiconductor Nanoparticles. Macromol Rapid Commun 2014;35:454-459.

[58] Bansal A, Kumar A, Kumar P, Bojja S, Chatterjee AK, Ray SS, Jain SL. Visible lightinduced surface initiated atom transfer radical polymerization of methyl methacrylate on titania/reduced graphene oxide nanocomposite. RSC Adv 2015;5:21189-21196.

[59] Cao Y, Xu Y, Zhang J, Yang D, Liu J. Well-controlled atom transfer radical polymerizations of acrylates using recyclable niobium complex nanoparticle as photocatalyst under visible light irradiation. Polymer 2015;61:198-203.

[60] Yan J, Li B, Zhou F, Liu W. Ultraviolet Light-Induced Surface-Initiated Atom-Transfer Radical Polymerization. ACS Macro Lett 2013;2:592-596.

[61] Ciftci M, Tasdelen MA, Yagci Y. Sunlight induced atom transfer radical polymerization by using dimanganese decacarbonyl. Polym Chem 2014;5:600-606.

[62] Wang JS, Matyjaszewski K. "Living"/Controlled Radical Polymerization. TransitionMetal-Catalyzed Atom Transfer Radical Polymerization in the Presence of a Conventional Radical Initiator. Macromolecules 1995;28:7572-7573. 
[63] Gromada J, Matyjaszewski K. Simultaneous Reverse and Normal Initiation in Atom Transfer Radical Polymerization. Macromolecules 2001;34:7664-7671.

[64] Arslan M, Yilmaz G, Yagci Y. Dibenzoyldiethylgermane as a visible light photoreducing agent for CuAAC click reactions. Polym Chem 2015;6:8168-8175.

[65] Guan Z, Smart B. A Remarkable Visible Light Effect on Atom-Transfer Radical Polymerization. Macromolecules 2000;33:6904-6906.

[66] Ribelli TG, Konkolewicz D, Bernhard S, Matyjaszewski K. How are radicals (re)generated in photochemical ATRP? J Am Chem Soc 2014;136:13303-13312.

[67] Anastasaki A, Nikolaou V, McCaul NW, Simula A, Godfrey J, Waldron C, Wilson P, Kempe K, Haddleton DM. Photoinduced Synthesis of $\alpha, \omega$-Telechelic SequenceControlled Multiblock Copolymers. Macromolecules 2015;48:1404-1411.

[68] Anastasaki A, Nikolaou V, Nurumbetov G, Truong NP, Pappas GS, Engelis NG, Quinn JF, Whittaker MR, Davis TP, Haddleton DM. Synthesis of Well-Defined Poly(acrylates) in Ionic Liquids via Copper(II)-Mediated Photoinduced Living Radical Polymerization. Macromolecules 2015;48:5140-5147.

[69] Anastasaki A, Nikolaou V, Simula A, Godfrey J, Li M, Nurumbetov G, Wilson P, Haddleton DM. Expanding the Scope of the Photoinduced Living Radical Polymerization of Acrylates in the Presence of CuBr 2 and Me 6 -Tren. Macromolecules 2014;47:38523859 .

[70] Chuang YM, Ethirajan A, Junkers T. Photoinduced Sequence-Controlled CopperMediated Polymerization: Synthesis of Decablock Copolymers. ACS Macro Lett 2014;3:732-737.

[71] Anastasaki A, Nikolaou V, Pappas GS, Zhang Q, Wan C, Wilson P, Davis TP, Whittaker MR, Haddleton DM. Photoinduced sequence-control via one pot living radical polymerization of acrylates. Chem Sci 2014;5:3536-3542.

[72] Nikolaou V, Anastasaki A, Alsubaie F, Simula A, Fox DJ, Haddleton DM. Copper(ii) gluconate (a non-toxic food supplement/dietary aid) as a precursor catalyst for effective photo-induced living radical polymerisation of acrylates. Polym Chem 2015;6:3581-3585.

[73] Anastasaki A, Nikolaou V, Brandford-Adams F, Nurumbetov G, Zhang Q, Clarkson GJ, Fox DJ, Wilson P, Kempe K, Haddleton DM. Photo-induced living radical polymerization of acrylates utilizing a discrete copper(II)-formate complex. Chem Commun 2015;51:5626-5629. 
[74] Mosnáček J, Eckstein-Andicsová A, Borská K. Ligand effect and oxygen tolerance studies in photochemically induced copper mediated reversible deactivation radical polymerization of methyl methacrylate in dimethyl sulfoxide. Polym Chem 2015;6:25232530.

[75] Chuang YM, Wenn B, Gielen S, Ethirajan A, Junkers T. Ligand switch in photoinduced copper-mediated polymerization: synthesis of methacrylate-acrylate block copolymers. Polym Chem 2015;6:6488-6497.

[76] Laun J, Vorobii M, de Los Santos Pereira A, Pop-Georgievski O, Trouillet V, Welle A, Barner-Kowollik C, Rodriguez-Emmenegger C, Junkers T. Surface Grafting via PhotoInduced Copper-Mediated Radical Polymerization at Extremely Low Catalyst Concentrations. Macromol Rapid Commun 2015;36:1681-1686.

[77] Mosnacek J, Kundys A, Andicsova A. Reversible-Deactivation Radical Polymerization of Methyl Methacrylate Induced by Photochemical Reduction of Various Copper Catalysts. Polymers 2014;6:2862-2874.

[78] Yang Q, Dumur F, Morlet-Savary F, Poly J, Lalevée J. Photocatalyzed Cu-Based ATRP Involving an Oxidative Quenching Mechanism under Visible Light. Macromolecules 2015;48:1972-1980.

[79] Wang GX, Lu M, Hou ZH, Yang CA, Liang EX, Liu Lc, Wu H, Li XL, Xu YX. Photoinduced atom transfer radical polymerization in ionic liquid. J Polym Res 2015;22:60/1-6.

[80] Zhang T, Chen T, Amin I, Jordan R. ATRP with a light switch: photoinduced ATRP using a household fluorescent lamp. Polym Chem 2014;5:4790-4796.

[81] Chantasirichot S, Inoue Y, Ishihara K. Photoinduced atom transfer radical polymerization in a polar solvent to synthesize a water-soluble poly(2-methacryloyloxyethyl phosphorylcholine) and its block-type copolymers. Polymer 2015;61:55-60.

[82] Vorobii M, de los Santos Pereira A, Pop-Georgievski O, Kostina NY, RodriguezEmmenegger C, Percec V. Synthesis of non-fouling polyN-(2hydroxypropyl)methacrylamide brushes by photoinduced SET-LRP. Polym Chem 2015;6:4210-4220.

[83] Amado E, Kressler J. Reversible Complexation of Iminophenylboronates with Mono- and Dihydroxy Methacrylate Monomers and Their Polymerization at Low Temperature by Photoinduced ATRP in One Pot. Macromolecules 2016;49:1532-1544. 
[84] Frick E, Anastasaki A, Haddleton DM, Barner-Kowollik C. Enlightening the Mechanism of Copper Mediated photordrp via High-Resolution Mass Spectrometry. J Am Chem Soc 2015;137:6889-6896.

[85] Larsson E, Pendergraph SA, Kaldeus T, Malmstrom E, Carlmark A. Cellulose grafting by photoinduced controlled radical polymerisation. Polym Chem 2015;6:1865-1874.

[86] Wenn B, Conradi M, Carreiras AD, Haddleton DM, Junkers T. Photo-induced coppermediated polymerization of methyl acrylate in continuous flow reactors. Polym Chem 2014;5:3053-3060.

[87] Pan X, Malhotra N, Simakova A, Wang Z, Konkolewicz D, Matyjaszewski K. Photoinduced Atom Transfer Radical Polymerization with ppm-Level Cu Catalyst by Visible Light in Aqueous Media. J Am Chem Soc 2015;137:15430-15433.

[88] Fantin M, Isse AA, Gennaro A, Matyjaszewski K. Understanding the Fundamentals of Aqueous ATRP and Defining Conditions for Better Control. Macromolecules 2015;48:6862-6875.

[89] Konkolewicz D, Magenau AJD, Averick SE, Simakova A, He H, Matyjaszewski K. ICAR ATRP with ppm Cu Catalyst in Water. Macromolecules 2012;45:4461-4468.

[90] Simakova A, Averick SE, Konkolewicz D, Matyjaszewski K. Aqueous ARGET ATRP. Macromolecules 2012;45:6371-6379.

[91] Jones GR, Whitfield R, Anastasaki A, Haddleton DM. Aqueous Copper(II) Photoinduced Polymerization of Acrylates: Low Copper Concentration and the Importance of Sodium Halide Salts. J Am Chem Soc 2016 DOI: 10.1021/jacs.6b02701

[92] Kwak Y, Matyjaszewski K. Photoirradiated Atom Transfer Radical Polymerization with an Alkyl Dithiocarbamate at Ambient Temperature. Macromolecules 2010;43:5180-5183.

[93] Ribelli TG, Konkolewicz D, Pan X, Matyjaszewski K. Contribution of Photochemistry to Activator Regeneration in ATRP. Macromolecules 2014;47:6316-6321.

[94] Zhang T, Gieseler D, Jordan R. Lights on! A significant photoenhancement effect on ATRP by ambient laboratory light. Polym Chem 2016;7:775-779.

[95] Zhao QL, Liu EH, Wang GX, Hou ZH, Zhan XH, Liu Lc, Wu H. Photoinduced ICAR ATRP of Methyl Methacrylate with AIBN as Photoinitiator. J Polym Res 2014;21:1-6.

[96] Wang GX, Lu M, Hou ZH, Gao Y, Liu LC, Wu H. Photoirradiated Fe-Mediated AGET ATRP of Methyl Methacrylate in the Presence of Alcohol. J Macromol Sci Part A 2014;51:565-571. 
[97] Liu Lc, Lu M, Hou ZH, Wang GX, Yang CA, Liang EX, Wu H, Li XL, Xu YX. PhotoInduced atom transfer radical polymerization with nanosized $\alpha-\mathrm{Fe} 2 \mathrm{O} 3$ as photoinitiator. $\mathrm{J}$ Appl Polym Sci 2015;132:42389/1-8.

[98] Bansal A, Kumar P, Sharma CD, Ray SS, Jain SL. Light-induced controlled free radical polymerization of methacrylates using iron-based photocatalyst in visible light. J Polym Sci Part A Polym Chem 2015;53:2739-2746.

[99] Telitel S, Dumur F, Campolo D, Poly J, Gigmes D, Pierre Fouassier J, Lalevée J. Iron complexes as potential photocatalysts for controlled radical photopolymerizations: A tool for modifications and patterning of surfaces. J Polym Sci Part A Polym Chem 2016;54:702-713.

[100] Pan X, Malhotra N, Zhang J, Matyjaszewski K. Photoinduced Fe-Based Atom Transfer Radical Polymerization in the Absence of Additional Ligands, Reducing Agents, and Radical Initiators. Macromolecules 2015;48:6948-6954.

[101] Xue Z, He D, Xie X. Iron-catalyzed atom transfer radical polymerization. Polym Chem 2015;6:1660-1687.

[102] Poli R, Allan LEN, Shaver MP. Iron-mediated reversible deactivation controlled radical polymerization. Prog Polym Sci 2014;39:1827-1845.

[103] Xue Z, Linh NTB, Noh SK, Lyoo WS. Phosphorus-Containing Ligands for Iron(III)Catalyzed Atom Transfer Radical Polymerization. Angew Chem Int Ed 2008;47:64266429.

[104] Wang Y, Kwak Y, Matyjaszewski K. Enhanced Activity of ATRP Fe Catalysts with Phosphines Containing Electron Donating Groups. Macromolecules 2012;45:5911-5915.

[105] Ando T, Kamigaito M, Sawamoto M. Iron(II) Chloride Complex for Living Radical Polymerization of Methyl Methacrylate. Macromolecules 1997;30:4507-4510.

[106] Matyjaszewski K, Wei M, Xia J, McDermott NE. Controlled/“Living” Radical Polymerization of Styrene and Methyl Methacrylate Catalyzed by Iron Complexes. Macromolecules 1997;30:8161-8164.

[107] Simakova A, Mackenzie M, Averick SE, Park S, Matyjaszewski K. Bioinspired IronBased Catalyst for Atom Transfer Radical Polymerization. Angew Chem Int Ed 2013;52:12148-12151.

[108] Wang Y, Matyjaszewski K. ATRP of MMA in Polar Solvents Catalyzed by FeBr2 without Additional Ligand. Macromolecules 2010;43:4003-4005. 
[109] Meyer TJ. Chemical approaches to artificial photosynthesis. Acc Chem Res 1989;22:163170.

[110] Lalrempuia R, McDaniel ND, Müller-Bunz H, Bernhard S, Albrecht M. Water Oxidation Catalyzed by Strong Carbene-Type Donor-Ligand Complexes of Iridium. Angew Chem Int Ed 2010;49:9765-9768.

[111] DiSalle BF, Bernhard S. Orchestrated Photocatalytic Water Reduction Using SurfaceAdsorbing Iridium Photosensitizers. J Am Chem Soc 2011;133:11819-11821.

[112] Kalyanasundaram K. Photophysics, photochemistry and solar energy conversion with tris(bipyridyl)ruthenium(II) and its analogues. Coord Chem Rev 1982;46:159-244.

[113] Howerton BS, Heidary DK, Glazer EC. Strained Ruthenium Complexes Are Potent Light-Activated Anticancer Agents. J Am Chem Soc 2012;134:8324-8327.

[114] Gualandi A, Marchini M, Mengozzi L, Natali M, Lucarini M, Ceroni P, Cozzi PG. Organocatalytic Enantioselective Alkylation of Aldehydes with Fe(bpy)3Br2 Catalyst and Visible Light. ACS Catalysis 2015;5:5927-5931.

[115] Nicewicz DA, MacMillan DWC. Merging Photoredox Catalysis with Organocatalysis: The Direct Asymmetric Alkylation of Aldehydes. Science 2008;322:77-80.

[116] Skubi KL, Yoon TP. Organic chemistry: Shape control in reactions with light. Nature 2014;515:45-46.

[117] Wallentin CJ, Nguyen JD, Finkbeiner P, Stephenson CRJ. Visible Light-Mediated Atom Transfer Radical Addition via Oxidative and Reductive Quenching of Photocatalysts. J Am Chem Soc 2012;134:8875-8884.

[118] Nguyen JD, Tucker JW, Konieczynska MD, Stephenson CRJ. Intermolecular Atom Transfer Radical Addition to Olefins Mediated by Oxidative Quenching of Photoredox Catalysts. J Am Chem Soc 2011;133:4160-4163.

[119] Fors BP, Hawker CJ. Control of a Living Radical Polymerization of Methacrylates by Light. Angew Chem Int Ed 2012;51:8850-8853.

[120] Lalevée J, Blanchard N, Tehfe MA, Peter M, Morlet-Savary F, Fouassier JP. A Novel Photopolymerization Initiating System Based on an Iridium Complex Photocatalyst. Macromol Rapid Commun 2011;32:917-920.

[121] Ma W, Chen H, Ma Y, Zhao C, Yang W. Visible-Light-Induced Controlled Polymerization of Hydrophilic Monomers with $\operatorname{Ir}(\mathrm{ppy}) 3$ as a Photoredox Catalyst in Anisole. Macromol Chem Phys 2014;215:1012-1021. 
[122] Liu Q, Liu L, Ma Y, Zhao C, Yang W. Visible light-induced controlled radical polymerization of methacrylates with perfluoroalkyl iodide as the initiator in conjugation with a photoredox catalyst fac-Ir(ppy)3. J Polym Sci Part A Polym Chem 2014;52:32833291.

[123] Ma W, Chen D, Wang L, Ma Y, Zhao C, Yang W. Visible Light-Controlled Radical Polymerization of Propargyl Methacrylate Activated by a Photoredox Catalyst facIr(ppy)3. J Macromol Sci Part A 2015;52:761-769.

[124] Treat NJ, Fors BP, Kramer JW, Christianson M, Chiu CY, Alaniz JRd, Hawker CJ. Controlled Radical Polymerization of Acrylates Regulated by Visible Light. ACS Macro Lett 2014;3:580-584.

[125] Muehlebach A, Rime F. Synthesis of well-defined macromonomers and comb copolymers from polymers made by atom transfer radical polymerization. J Polym Sci Part A Polym Chem 2003;41:3425-3439.

[126] Telitel S, Dumur F, Telitel S, Soppera O, Lepeltier M, Guillaneuf Y, Poly J, MorletSavary F, Fioux P, Fouassier JP, Gigmes D, Lalevée J. Photoredox catalysis using a new iridium complex as an efficient toolbox for radical, cationic and controlled polymerizations under soft blue to green lights. Polym Chem 2015;6:613-624.

[127] Iwai K, Uesugi M, Takemura F. Tris(2,2prime-bipyridine)Ruthenium(II)-Sensitized Photopolymerization of Acrylamide. Polym J 1985;17:1005-1011.

[128] Zhang G, Song IY, Ahn KH, Park T, Choi W. Free Radical Polymerization Initiated and Controlled by Visible Light Photocatalysis at Ambient Temperature. Macromolecules 2011;44:7594-7599.

[129] Alfredo NV, Jalapa NE, Morales SL, Ryabov AD, Le Lagadec R, Alexandrova L. LightDriven Living/Controlled Radical Polymerization of Hydrophobic Monomers Catalyzed by Ruthenium(II) Metalacycles. Macromolecules 2012;45:8135-8146.

[130] Ryabov AD, Le Lagadec R, Estevez H, Toscano RA, Hernandez S, Alexandrova L, Kurova VS, Fischer A, Sirlin C, Pfeffer M. Synthesis, Characterization, and Electrochemistry of Biorelevant Photosensitive Low-Potential Orthometalated Ruthenium Complexes. Inorg Chem 2005;44:1626-1634.

[131] Nzulu F, Telitel S, Stoffelbach F, Graff B, Morlet-Savary F, Lalevee J, Fensterbank L, Goddard JP, Ollivier C. A dinuclear gold(i) complex as a novel photoredox catalyst for light-induced atom transfer radical polymerization. Polym Chem 2015;6:4605-4611. 
[132] Miyake GM, Theriot JC. Perylene as an Organic Photocatalyst for the Radical Polymerization of Functionalized Vinyl Monomers through Oxidative Quenching with Alkyl Bromides and Visible Light. Macromolecules 2014;47:8255-8261.

[133] Liu XD, Zhang LF, Cheng ZP, Zhu XL. Metal-free photoinduced electron transfer-atom transfer radical polymerization (PET-ATRP) via a visible light organic photocatalyst. Polym Chem 2016;7:689-700.

[134] Theriot JC, Lim C-H, Yang H, Ryan MD, Musgrave CB, Miyake GM. Organocatalyzed atom transfer radical polymerization driven by visible light. Science 2016;352:10821086.

[135] Kowalewski T, Tsarevsky NV, Matyjaszewski K. Nanostructured Carbon Arrays from Block Copolymers of Polyacrylonitrile. J Am Chem Soc 2002;124:10632-10633.

[136] McGann JP, Zhong M, Kim EK, Natesakhawat S, Jaroniec M, Whitacre JF, Matyjaszewski K, Kowalewski T. Block Copolymer Templating as a Path to Porous Nanostructured Carbons with Highly Accessible Nitrogens for Enhanced (Electro)chemical Performance. Macromol Chem Phys 2012;213:1078-1090.

[137] Zhong M, Natesakhawat S, Baltrus JP, Luebke D, Nulwala H, Matyjaszewski K, Kowalewski T. Copolymer-templated nitrogen-enriched porous nanocarbons for $\mathrm{CO} 2$ capture. Chem Commun 2012;48:11516-11518.

[138] Zhong M, Kim EK, McGann JP, Chun SE, Whitacre JF, Jaroniec M, Matyjaszewski K, Kowalewski T. Electrochemically Active Nitrogen-Enriched Nanocarbons with WellDefined Morphology Synthesized by Pyrolysis of Self-Assembled Block Copolymer. J Am Chem Soc 2012;134:14846-14857.

[139] Matyjaszewski K, Jo SM, Paik Hj, Shipp DA. An Investigation into the CuX/2,2‘Bipyridine $(\mathrm{X}=\mathrm{Br}$ or $\mathrm{Cl}$ ) Mediated Atom Transfer Radical Polymerization of Acrylonitrile. Macromolecules 1999;32:6431-6438.

[140] Matyjaszewski K, Mu Jo S, Paik Hj, Gaynor SG. Synthesis of Well-Defined Polyacrylonitrile by Atom Transfer Radical Polymerization. Macromolecules 1997;30:6398-6400.

[141] Pan X, Fang C, Fantin M, Malhotra N, So WY, Peteanu LA, Isse AA, Gennaro A, Liu P, Matyjaszewski K. Mechanism of Photoinduced Metal-Free Atom Transfer Radical Polymerization: Experimental and Computational Studies. J Am Chem Soc 2016;138:2411-2425. 
[142] Kaur A, Ribelli TG, Schröder K, Matyjaszewski K, Pintauer T. Properties and ATRP Activity of Copper Complexes with Substituted Tris(2-pyridylmethyl)amine-Based Ligands. Inorg Chem 2015;54:1474-1486.

[143] Lin CY, Coote ML, Gennaro A, Matyjaszewski K. Ab Initio Evaluation of the Thermodynamic and Electrochemical Properties of Alkyl Halides and Radicals and Their Mechanistic Implications for Atom Transfer Radical Polymerization. J Am Chem Soc 2008;130:12762-12774.

[144] Isse AA, Bortolamei N, De Paoli P, Gennaro A. On the mechanism of activation of copper-catalyzed atom transfer radical polymerization. Electrochim Acta 2013;110:655662.

[145] Marcus RA. On the Theory of Oxidation- Reduction Reactions Involving Electron Transfer. I. J Chem Phys 1956;24:966-978.

[146] Pause L, Robert M, Savéant JM. Reductive Cleavage of Carbon Tetrachloride in a Polar Solvent. An Example of a Dissociative Electron Transfer with Significant Attractive Interaction between the Caged Product Fragments. J Am Chem Soc 2000;122:9829-9835.

[147] Saveant JM. A simple model for the kinetics of dissociative electron transfer in polar solvents. Application to the homogeneous and heterogeneous reduction of alkyl halides. $\mathbf{J}$ Am Chem Soc 1987;109:6788-6795.

[148] Saveant JM. Dissociative electron transfer. New tests of the theory in the electrochemical and homogeneous reduction of alkyl halides. J Am Chem Soc 1992;114:10595-10602.

[149] Debuigne A, Hurtgen M, Detrembleur C, Jérôme C, Barner-Kowollik C, Junkers T. Interpolymer radical coupling: A toolbox complementary to controlled radical polymerization. Prog Polym Sci 2012;37:1004-1030.

[150] Wenn B, Martens AC, Chuang YM, Gruber J, Junkers T. Efficient multiblock star polymer synthesis from photo-induced copper-mediated polymerization with up to 21 arms. Polym Chem 2016;7:2720-2727.

[151] Roy RK, Meszynska A, Laure C, Charles L, Verchin C, Lutz JF. Design and synthesis of digitally encoded polymers that can be decoded and erased. Nat Commun 2015;6:7237/18.

[152] Vandenbergh J, Reekmans G, Adriaensens P, Junkers T. Synthesis of sequence controlled acrylate oligomers via consecutive RAFT monomer additions. Chem Commun 2013;49:10358-10360. 
[153] Vandenbergh J, Reekmans G, Adriaensens P, Junkers T. Synthesis of sequence-defined acrylate oligomers via photo-induced copper-mediated radical monomer insertions. Chem Sci 2015;6:5753-5761.

[154] Adali-Kaya Z, Tse Sum Bui B, Falcimaigne-Cordin A, Haupt K. Molecularly imprinted polymer nanomaterials and nanocomposites: atom-transfer radical polymerization with acidic monomers. Angew Chem Int Ed 2015;54:5192-5195.

[155] Junkers T, Wenn B. Continuous photoflow synthesis of precision polymers. React Chem Eng 2016;1:60-64.

[156] Elliott LD, Knowles JP, Koovits PJ, Maskill KG, Ralph MJ, Lejeune G, Edwards LJ, Robinson RI, Clemens IR, Cox B, Pascoe DD, Koch G, Eberle M, Berry MB, BookerMilburn KI. Batch versus flow photochemistry: a revealing comparison of yield and productivity. Chemistry 2014;20:15226-15232.

[157] Conradi M, Junkers T. Fast and Efficient $2+2$ UV Cycloaddition for Polymer Modification via Flow Synthesis. Macromolecules 2014;47:5578-5585.

[158] Conradi M, Junkers T. Efficient 2+2 photocycloadditions under equimolar conditions by employing a continuous UV-flow reactor. J Photochem Photobiol A 2013;259:41-46.

[159] Kermagoret A, Wenn B, Debuigne A, Jerome C, Junkers T, Detrembleur C. Improved photo-induced cobalt-mediated radical polymerization in continuous flow photoreactors. Polym Chem 2015;6:3847-3857.

[160] Melker A, Fors BP, Hawker CJ, Poelma JE. Continuous flow synthesis of poly(methyl methacrylate) via a light-mediated controlled radical polymerization. J Polym Sci Part A Polym Chem 2015;53:2693-2698.

[161] Otsu T, Matsunaga T, Doi T, Matsumoto A. Features of living radical polymerization of vinyl monomers in homogeneous system using $\mathrm{N}, \mathrm{N}$-diethyldithiocarbamate derivatives as photoiniferters. Eur Polym J 1995;31:67-78.

[162] Otsu T, Yoshida M, Tazaki T. A model for living radical polymerization. Makromol. 1982;3:133-140.

[163] Tasdelen MA, Durmaz YY, Karagoz B, Bicak N, Yagci Y. A new photoiniferter/RAFT agent for ambient temperature rapid and well-controlled radical polymerization. J Polym Sci Part A Polym Chem 2008;46:3387-3395.

[164] Kwon TS, Ochiai H, Kondo S, Takagi K, Kunisada H, Yuki Y. Radical polymerization of p-substituted styrenes with benzyl phenyl selenide as photoiniferter. Polym J

1999;31:411-417. 
[165] Bertin D, Boutevin B, Gramain P, Fabre JM, Montginoul C. Living radical polymerization of methyl methacrylate in the presence of piperidino-dithiocarbamate derivatives as photo-iniferters. Eur Polym J 1998;34:85-90.

[166] Yang XM, Qiu KY. Synthesis of block copolymers by radical polymerization of endfunctional polystyrene. J Appl Polym Sci 1997;64:1169-1174.

[167] Doi T, Matsumoto A, Otsu T. Radical polymerization of methyl acrylate by use of benzyl $\mathrm{N}, \mathrm{N}$-diethyldithiocrbamate in combination with tetraethylthiuram disulfide as a twocomponent iniferter. J Polym Sci Part A Polym Chem 1994;32:2911-2918.

[168] Rathore K, Reddy KR, Tomer NS, Desai SM, Singh RP. Visible-light-induced controlled/living radical polymerization of styrene with a phenyl seleno group at one terminal chain end: 1-(phenylseleno)ethyl Benzene as a photoiniferter. J Appl Polym Sci 2004;93:348-355.

[169] Kwon TS, Suzuki K, Takagi K, Kunisada H, Yuki Y. Radical polymerization of methyl methacrylate with diphenyl diselenide under thermal or photoirradiational conditions. $\mathbf{J}$ Macromol Sci Pure Appl Chem 2001;A38:591-604.

[170] Poljansek I, Kozamernik T, Sebenik A. Free radical polymerization of styrene and methyl methacrylate with biphosphine. Kovine, Zlitine, Tehnol 1997;31:81-84.

[171] Lalevee J, Blanchard N, ElRoz M, Allonas X, Fouassier JP. New Photoiniferters: Respective Role of the Initiating and Persistent Radicals. Macromolecules 2008;41:23472352.

[172] Guillaneuf Y, Bertin D, Gigmes D, Versace DL, Lalevee J, Fouassier JP. Toward Nitroxide-Mediated Photopolymerization. Macromolecules 2010;43:2204-2212.

[173] Durmaz YY, Karagoz B, Bicak N, Yagci Y. Synthesis of block copolymers by combination of ATRP and photoiniferter processes. Polym Int 2008;57:1182-1187.

[174] Kwon TS, Takagi K, Kunisada H, Yuki Y. Synthesis of ABA type triblock copolymers by radical polymerization with 1,4-Bis(p-tert-butylphenylselenomethyl)benzene as a photoiniferter. J Macromol Sci Pure Appl Chem 2001;A38:605-626.

[175] Kuriyama A, Otsu T. Living radical polymerization of methyl methacrylate with a tetrafunctional photoiniferter: synthesis of a star polymer. Polym J 1984;16:511-514.

[176] Quinn JF, Barner L, Barner-Kowollik C, Rizzardo E, Davis TP. Reversible additionfragmentation chain transfer polymerization initiated with ultraviolet radiation. Macromolecules 2002;35:7620-7627. 
[177] Lu L, Yang N, Cai Y. Well-controlled reversible addition-fragmentation chain transfer radical polymerization under ultraviolet radiation at ambient temperature. Chem Commun 2005 :5287-5288.

[178] Lu L, Zhang H, Yang N, Cai Y. Toward Rapid and Well-Controlled Ambient Temperature RAFT Polymerization under UV-Vis Radiation: Effect of Radiation Wave Range. Macromolecules 2006;39:3770-3776.

[179] Buback M, Egorov M, Junkers T, Panchenko E. Free-radical termination kinetics studied using a novel SP-PLP-ESR technique. Macromol Rapid Commun 2004;25:1004-1009.

[180] Buback M, Hesse P, Junkers T, Theis T, Vana P. Chain-Length-Dependent Termination in Acrylate Radical Polymerization Studied via Pulsed-Laser-Initiated RAFT Polymerization. Aust J Chem 2007;60:779-787.

[181] Buback M, Junkers T, Vana P. Laser single pulse initiated RAFT polymerization for assessing chain-length dependent radical termination kinetics. Macromol Rapid Commun 2005;26:796-802.

[182] Junkers T, Theis A, Buback M, Davis TP, Stenzel MH, Vana P, Barner-Kowollik C. Chain Length Dependent Termination in Butyl Acrylate Free-Radical Polymerization Studied via Stationary and Pulsed Laser Initiated RAFT Polymerization. Macromolecules 2005;38:9497-9508.

[183] Buback M, Junkers T, Vana P. Pulsed-laser initiated reversible addition fragmentation chain transfer polymerization. ACS Symp Ser 2006;944:455-472.

[184] Barth J, Buback M, Meiser W, Vana P. Easy Access to the RAFT Equilibrium Constant. Macromolecules 2010;43:51-54.

[185] Meiser W, Buback M, Barth J, Vana P. RAFT/MADIX rate coefficients measured via time-resolved EPR spectroscopy after pulse laser initiation. Polymer 2010;51:5977-5982.

[186] Meiser W, Buback M, Sidoruk A. EPR Investigations into the Kinetics of Trithiocarbonate-Mediated RAFT-Polymerization of Butyl Acrylate. Macromol Chem Phys 2013;214:2108-2114.

[187] Buback M, Hesse P, Junkers T, Vana P. Determination of addition and fragmentation rate coefficients in RAFT polymerization via time-resolved ESR spectroscopy after laser pulse initiation. Macromol Rapid Commun 2006;27:182-187.

[188] Chernikova E, Golubev V, Filippov A, Lin CY, Coote ML. Use of spin traps to measure the addition and fragmentation rate coefficients of small molecule RAFT-adduct radicals. Polym Chem 2010;1:1437-1440. 
[189] Junkers T. RAFT kinetics revisited: Revival of the RAFT debate. J Polym Sci Part A Polym Chem 2011;49:4154-4163.

[190] Ranieri K, Delaittre G, Barner-Kowollik C, Junkers T. Direct Access to Dithiobenzoate RAFT Agent Fragmentation Rate Coefficients by ESR Spin-Trapping. Macromol Rapid Commun 2014;35:2023-2028.

[191] Zhang H, Deng J, Lu L, Cai Y. Ambient-Temperature RAFT Polymerization of Styrene and Its Functional Derivatives under Mild Long-Wave UV-vis Radiation.

Macromolecules 2007;40:9252-9261.

[192] Jiang W, Lu L, Cai Y. Highly efficient and well-controlled ambient temperature RAFT polymerization under solar radiation. Macromol Rapid Commun 2007;28:725-728.

[193] Yin H, Zheng H, Lu L, Liu P, Cai Y. Highly efficient and well-controlled ambient temperature RAFT polymerization of glycidyl methacrylate under visible light radiation. J Polym Sci Part A Polym Chem 2007;45:5091-5102.

[194] Shi Y, Liu G, Gao H, Lu L, Cai Y. Effect of Mild Visible Light on Rapid Aqueous RAFT Polymerization of Water-Soluble Acrylic Monomers at Ambient Temperature: Initiation and Activation. Macromolecules 2009;42:3917-3926.

[195] Bouchekif H, Narain R. Reversible addition-fragmentation chain transfer polymerization of N-isopropylacrylamide: a comparison between a conventional and a fast initiator. $\mathrm{J}$ Phys Chem B 2007;111:11120-11126.

[196] Ham Mk, HoYouk J, Kwon YK, Kwark YJ. Photoinitiated RAFT polymerization of vinyl acetate. J Polym Sci Part A Polym Chem 2012;50:2389-2397.

[197] Shim SH, Ham Mk, Huh J, Kwon YK, Kwark YJ. Simultaneous control over the molecular weight and tacticity of poly(vinyl acetate) using a low-temperature photoinitiated RAFT process in fluoroalcohols. Polym Chem 2013;4:5449-5455.

[198] Kwak Y, Nicolay R, Matyjaszewski K. Synergistic Interaction Between ATRP and RAFT: Taking the Best of Each World. Aust J Chem 2009;62:1384-1401.

[199] Kwak Y, Nicolay R, Matyjaszewski K. Concurrent ATRP/RAFT of styrene and methyl methacrylate with dithioesters catalyzed by copper(I) complexes. Macromolecules 2008;41:6602-6604.

[200] Nicolay R, Kwak Y, Matyjaszewski K. A Green Route to Well-Defined High-MolecularWeight (Co)polymers Using ARGET ATRP with Alkyl Pseudohalides and Copper Catalysis. Angew Chem Int Ed 2010;49:541-544. 
[201] Muthukrishnan S, Pan EH, Stenzel MH, Barner-Kowollik C, Davis TP, Lewis D, Barner L. Ambient Temperature RAFT Polymerization of Acrylic Acid Initiated with Ultraviolet Radiation in Aqueous Solution. Macromolecules 2007;40:2978-2980.

[202] Motokucho S, Sudo A, Endo T. Polymer having a trithiocarbonate moiety in the main chain: application to reversible addition-fragmentation chain transfer controlled thermal and photoinduced monomer insertion polymerizations. J Polym Sci Part A Polym Chem 2006;44:6324-6331.

[203] Ran R, Yu Y, Wan T. Photoinitiated RAFT polymerization in the presence of trithiocarbonate. J Appl Polym Sci 2007;105:398-404.

[204] Ran R, Wan T, Gao T, Gao J, Chen Z. Controlled free radical photopolymerization of styrene initiated by trithiocarbonate. Polym Int 2008;57:28-34.

[205] Khan MY, Cho MS, Kwark YJ. Dual Roles of a Xanthate as a Radical Source and Chain Transfer Agent in the Photoinitiated RAFT Polymerization of Vinyl Acetate.

Macromolecules 2014;47:1929-1934.

[206] Wang H, Li Q, Dai J, Du F, Zheng H, Bai R. Real-Time and in Situ Investigation of "Living"/Controlled Photopolymerization in the Presence of a Trithiocarbonate. Macromolecules 2013;46:2576-2582.

[207] Poly J, Cabannes-Boue B, Hebinger L, Mangin R, Sauvage A, Xiao P, Morlet-Savary F, Lalevee J. Polymers synthesized by RAFT as versatile macrophotoinitiators. Polym Chem 2015;6:5766-5772.

[208] McKenzie TG, Fu Q, Wong EHH, Dunstan DE, Qiao GG. Visible Light Mediated Controlled Radical Polymerization in the Absence of Exogenous Radical Sources or Catalysts. Macromolecules 2015;48:3864-3872.

[209] McKenzie TG, Wong EHH, Fu Q, Sulistio A, Dunstan DE, Qiao GG. Controlled Formation of Star Polymer Nanoparticles via Visible Light Photopolymerization. ACS Macro Lett 2015;4:1012-1016.

[210] Amamoto Y, Kamada J, Otsuka H, Takahara A, Matyjaszewski K. Repeatable Photoinduced Self-Healing of Covalently Cross-Linked Polymers through Reshuffling of Trithiocarbonate Units. Angew Chem Int Ed 2011;50:1660-1663.

[211] Amamoto Y, Otsuka H, Takahara A, Matyjaszewski K. Self-Healing of Covalently Cross-Linked Polymers by Reshuffling Thiuram Disulfide Moieties in Air under Visible Light. Adv Mater 2012;24:3975-3980. 
[212] Xu J, Shanmugam S, Corrigan NA, Boyer C. Catalyst-Free Visible Light-Induced RAFT Photopolymerization. In: Matyjaszewski K, Sumerlin BS, Tsarevsky NV, Chiefari J, editors. Controlled Radical Polymerization: Mechanisms. Washington DC: American Chemical Society, 2015. p. 247-267.

[213] Yeow J, Sugita OR, Boyer C. Visible Light-Mediated Polymerization-Induced SelfAssembly in the Absence of External Catalyst or Initiator. ACS Macro Lett 2016;5:558564.

[214] Xu JT, Jung K, Atme A, Shanmugam S, Boyer C. A Robust and Versatile Photoinduced Living Polymerization of Conjugated and Unconjugated Monomers and Its Oxygen Tolerance. J Am Chem Soc 2014;136:5508-5519.

[215] Fors BP, Poelma JE, Menyo MS, Robb MJ, Spokoyny DM, Kramer JW, Waite JH, Hawker CJ. Fabrication of Unique Chemical Patterns and Concentration Gradients with Visible Light. J Am Chem Soc 2013;135:14106-14109.

[216] Shanmugam S, Xu J, Boyer C. Photoinduced Electron Transfer-Reversible AdditionFragmentation Chain Transfer (PET-RAFT) Polymerization of Vinyl Acetate and NVinylpyrrolidinone: Kinetic and Oxygen Tolerance Study. Macromolecules 2014;47:4930-4942.

[217] Fu C, Xu J, Tao L, Boyer C. Combining Enzymatic Monomer Transformation with Photoinduced Electron Transfer - Reversible Addition-Fragmentation Chain Transfer for the Synthesis of Complex Multiblock Copolymers. ACS Macro Lett 2014;3:633-638.

[218] Xu J, Jung K, Boyer C. Oxygen Tolerance Study of Photoinduced Electron TransferReversible Addition-Fragmentation Chain Transfer (PET-RAFT) Polymerization Mediated by Ru(bpy)3Cl2. Macromolecules 2014;47:4217-4229.

[219] Shanmugam S, Boyer C. Stereo-, Temporal and Chemical Control through Photoactivation of Living Radical Polymerization: Synthesis of Block and Gradient Copolymers. J Am Chem Soc 2015;137:9988-9999.

[220] Xu J, Shanmugam S, Duong HT, Boyer C. Organo-photocatalysts for photoinduced electron transfer-reversible addition-fragmentation chain transfer (PET-RAFT) polymerization. Polym Chem 2015;6:5615-5624.

[221] Xu J, Shanmugam S, Boyer C. Organic Electron Donor-Acceptor Photoredox Catalysts: Enhanced Catalytic Efficiency toward Controlled Radical Polymerization. ACS Macro Lett 2015;4:926-932. 
[222] Shanmugam S, Xu J, Boyer C. Utilizing the electron transfer mechanism of chlorophyll a under light for controlled radical polymerization. Chem Sci 2015;6:1341-1349.

[223] Shanmugam S, Xu J, Boyer C. Exploiting Metalloporphyrins for Selective Living Radical Polymerization Tunable over Visible Wavelengths. J Am Chem Soc 2015;137:9174-9185.

[224] Zhao J, Lalevee J, Lu H, MacQueen R, Kable SH, Schmidt TW, Stenzel MH, Xiao P. A new role of curcumin: as a multicolor photoinitiator for polymer fabrication under household UV to red LED bulbs. Polym Chem 2015;6:5053-5061.

[225] Miao X, Li J, Zhang Z, Cheng Z, Zhang W, Zhu J, Zhu X. Dimanganese decacarbonyl/2cyanoprop-2-yl-1-dithionaphthalate: toward sunlight induced RAFT polymerization of MMA. Polym Chem 2014;5:4641-4648.

[226] Zhang J, Li A, Liu H, Yang D, Liu J. Well-controlled RAFT polymerization initiated by recyclable surface-modified $\mathrm{Nb}(\mathrm{OH}) 5$ nanoparticles under visible light irradiation. $\mathrm{J}$

Polym Sci Part A Polym Chem 2014;52:2715-2724.

[227] Chen M, MacLeod MJ, Johnson JA. Visible-Light-Controlled Living Radical Polymerization from a Trithiocarbonate Iniferter Mediated by an Organic Photoredox Catalyst. ACS Macro Lett 2015;4:566-569.

[228] Shanmugam S, Xu J, Boyer C. Light-Regulated Polymerization under Near-Infrared/FarRed Irradiation Catalyzed by Bacteriochlorophyll a. Angew Chem Int Ed 2016;55:10361040 .

[229] Corrigan N, Xu J, Boyer C. A Photoinitiation System for Conventional and Controlled Radical Polymerization at Visible and NIR Wavelengths. Macromolecules 2016;49:3274-3285.

[230] Bagheri A, Yeow J, Arandiyan H, Xu J, Boyer C, Lim M. Polymerization of a Photocleavable Monomer Using Visible Light. Macromol Rapid Commun 2016;37:905910.

[231] Xu J, Shanmugam S, Fu C, Aguey-Zinsou K-F, Boyer C. Selective Photoactivation: From a Single Unit Monomer Insertion Reaction to Controlled Polymer Architectures. J Am Chem Soc 2016;138:3094-3106.

[232] Chen M, Johnson JA. Improving photo-controlled living radical polymerization from trithiocarbonates through the use of continuous-flow techniques. Chem Commun 2015;51:6742-6745. 
[233] Hook BDA, Dohle W, Hirst PR, Pickworth M, Berry MB, Booker-Milburn KI. A practical flow reactor for continuous organic photochemistry. J Org Chem 2005;70:7558-7564.

[234] Gardiner J, Hornung CH, Tsanaktsidis J, Guthrie D. Continuous flow photo-initiated RAFT polymerisation using a tubular photochemical reactor. Eur Polym J 2016 doi:10.1016/j.eurpolymj.2016.01.033

[235] Shim SE, Jung H, Lee H, Biswas J, Choe S. Living radical dispersion photopolymerization of styrene by a reversible addition-fragmentation chain transfer (RAFT) agent. Polymer 2003;44:5563-5572.

[236] Shim SE, Shin Y, Jun JW, Lee K, Jung H, Choe S. Living-Free-Radical Emulsion Photopolymerization of Methyl Methacrylate by a Surface Active Iniferter (Suriniferter). Macromolecules 2003;36:7994-8000.

[237] Tan J, Rao X, Wu X, Deng H, Yang J, Zeng Z. Photoinitiated RAFT Dispersion Polymerization: A Straightforward Approach toward Highly Monodisperse Functional Microspheres. Macromolecules 2012;45:8790-8795.

[238] Tan J, Zhao G, Zeng Z, Winnik MA. PMMA Microspheres with Embedded Lanthanide Nanoparticles by Photoinitiated Dispersion Polymerization with a Carboxy-Functional Macro-RAFT Agent. Macromolecules 2015;48:3629-3640.

[239] Tan J, Rao X, Jiang D, Yang J, Zeng Z. One-stage photoinitiated RAFT dispersion polymerization - Reaction parameters for achieving high particle size uniformity. Polymer 2014;55:2380-2388.

[240] Tan J, Rao X, Yang J, Zeng Z. Synthesis of Highly Monodisperse Surface-Functional Microspheres by Photoinitiated RAFT Dispersion Polymerization Using Macro-RAFT Agents. Macromolecules 2013;46:8441-8448.

[241] Tan J, Yu M, Rao X, Yang J, Zeng Z. Fast and facile one-step synthesis of monodisperse thermo-responsive core-shell microspheres and applications. Polym Chem 2015;6:66986708 .

[242] Tan J, Zhao G, Lu Y, Zeng Z, Winnik MA. Synthesis of PMMA Microparticles with a Narrow Size Distribution by Photoinitiated RAFT Dispersion Polymerization with a Macromonomer as the Stabilizer. Macromolecules 2014;47:6856-6866.

[243] Tan J, Rao X, Yang J, Zeng Z. Monodisperse highly cross-linked "living" microspheres prepared via photoinitiated RAFT dispersion polymerization. RSC Adv 2015;5:1892218931. 
[244] Jung K, Xu J, Zetterlund PB, Boyer C. Visible-Light-Regulated Controlled/Living Radical Polymerization in Miniemulsion. ACS Macro Lett 2015;4:1139-1143.

[245] Yeow J, Xu J, Boyer C. Polymerization-Induced Self-Assembly Using Visible Light Mediated Photoinduced Electron Transfer-Reversible Addition-Fragmentation Chain Transfer Polymerization. ACS Macro Lett 2015;4:984-990.

[246] Tan J, Bai Y, Zhang X, Zhang L. Room temperature synthesis of poly(poly(ethylene glycol) methyl ether methacrylate)-based diblock copolymer nano-objects via Photoinitiated Polymerization-Induced Self-Assembly (Photo-PISA). Polym Chem 2016;7:2372-2380.

[247] Park HY, Kloxin CJ, Fordney MF, Bowman CN. Stress relaxation of trithiocarbonatedimethacrylate-based dental composites. Dent Mater 2012;28:888-893.

[248] Amamoto Y, Otsuka H, Takahara A, Matyjaszewski K. Changes in Network Structure of Chemical Gels Controlled by Solvent Quality through Photoinduced Radical Reshuffling Reactions of Trithiocarbonate Units. ACS Macro Lett 2012;1:478-481.

[249] Haddleton DM. Polymer Chemistry Rooftop reactions. Nature 2013;5:366-368.

[250] Zhou H, Johnson JA. Photo-controlled Growth of Telechelic Polymers and End-linked Polymer Gels. Angew Chem Int Ed 2013;52:2235-2238.

[251] Scaiano JC, Connolly TJ, Mohtat N, Pliva CN. Exploratory study of the quenching of photosensitizers by initiators of free radical "living" polymerization. Can J Chem 1997;75:92-97.

[252] Goto A, Scaiano JC, Maretti L. Photolysis of an alkoxyamine using intramolecular energy transfer from a quinoline antenna - towards photo-induced living radical polymerization. Photochem Photobio Sci 2007;6:833-835.

[253] Hu S, Malpert JH, Yang X, Neckers DC. Exploring chromophore tethered aminoethers as potential photoinitiators for controlled radical polymerization. Polymer 2000;41:445-452.

[254] Versace DL, Lalevee J, Fouassier JP, Gigmes D, Guillaneuf Y, Bertin D. Photosensitized Alkoxyamines as Bicomponent Radical Photoinitiators. J Polym Sci Part A Polym Chem 2010;48:2910-2915.

[255] Versace DL, Lalevee J, Fouassier JP, Guillaneuf Y, Bertin D, Gigmes D. Photodissociation Rate Constants of New Light Sensitive Alkoxyamines. Macromol Rapid Commun 2010;31:1383-1388. 
[256] Guillaneuf Y, Versace DL, Bertin D, Lalevee J, Gigmes D, Fouassier JP. Importance of the Position of the Chromophore Group on the Dissociation Process of Light Sensitive Alkoxyamines. Macromol Rapid Commun 2010;31:1909-1913.

[257] Versace DL, Guillaneuf Y, Bertin D, Fouassier JP, Lalevee J, Gigmes D. Structural effects on the photodissociation of alkoxyamines. Org Biomol Chem 2011;9:2892-2898.

[258] Morris J, Telitel S, Fairfull-Smith KE, Bottle SE, Lalevee J, Clement JL, Guillaneuf Y, Gigmes D. Novel polymer synthesis methodologies using combinations of thermally- and photochemically-induced nitroxide mediated polymerization. Polym Chem 2015;6:754763.

[259] Debuigne A, Poli R, Jérôme C, Jérôme R, Detrembleur C. Overview of cobalt-mediated radical polymerization: Roots, state of the art and future prospects. Prog Polym Sci 2009;34:211-239.

[260] Hurtgen M, Detrembleur C, Jerome C, Debuigne A. Insight into OrganometallicMediated Radical Polymerization. Polym Rev 2011;51:188-213.

[261] Peng CH, Yang TY, Zhao Y, Fu X. Reversible deactivation radical polymerization mediated by cobalt complexes: recent progress and perspectives. Org Biomol Chem 2014;12:8580-8587.

[262] Detrembleur C, Versace DL, Piette Y, Hurtgen M, J?r?me C, Lalev?e J, Debuigne A. Synthetic and mechanistic inputs of photochemistry into the bis-acetylacetonatocobaltmediated radical polymerization of n-butyl acrylate and vinyl acetate. Polym Chem 2012;3:1856-1866.

[263] Miao X, Zhu W, Zhang Z, Zhang W, Zhu X, Zhu J. Photo-induced cobalt-mediated radical polymerization of vinyl acetate. Polym Chem 2014;5:551-557.

[264] Zhao Y, Yu M, Fu X. Photo-cleavage of the cobalt?carbon bond: visible light-induced living radical polymerization mediated by organo-cobalt porphyrins. Chem Commun 2013;49:5186-5188.

[265] Zhao Y, Yu M, Zhang S, Liu Y, Fu X. Visible Light Induced Living/Controlled Radical Polymerization of Acrylates Catalyzed by Cobalt Porphyrins. Macromolecules 2014;47:6238-6245.

[266] Zhao Y, Yu M, Zhang S, Wu Z, Liu Y, Peng CH, Fu X. A well-defined, versatile photoinitiator (salen)Co?CO2CH3 for visible light-initiated living/controlled radical polymerization. Chem Sci 2015;6:2979-2988. 
[267] Zhao Y, Zhang S, Wu Z, Liu X, Zhao X, Peng CH, Fu X. Visible-Light-Induced Living Radical Polymerization (LRP) Mediated by (salen)Co(II)/TPO at Ambient Temperature. Macromolecules 2015;48:5132-5139.

[268] Matyjaszewski K, Gaynor S, Wang JS. Controlled Radical Polymerizations: The Use of Alkyl Iodides in Degenerative Transfer. Macromolecules 1995;28:2093-2095.

[269] David G, Boyer C, Tonnar J, Ameduri B, Lacroix-Desmazes P, Boutevin B. Use of Iodocompounds in Radical Polymerization. Chem Rev 2006;106:3936-3962.

[270] Koumura K, Satoh K, Kamigaito M. Mn-2(CO)(10)-Induced Controlled/Living Radical Copolymerization of Vinyl Acetate and Methyl Acrylate: Spontaneous Formation of Block Copolymers Consisting of Gradient and Homopolymer Segments. J Polym Sci Part A Polym Chem 2009;47:1343-1353.

[271] Koumura K, Satoh K, Kamigaito M. Mn-2(CO)(10)-Induced Controlled/Living Radical Copolymerization of Methyl Acrylate and 1-Hexene in Fluoroalcohol: High alpha-Olefin Content Copolymers with Controlled Molecular Weights. Macromolecules 2009;42:2497-2504.

[272] Koumura K, Satoh K, Kamigaito M. Manganese-Based Controlled/Living Radical Polymerization of Vinyl Acetate, Methyl Acrylate, and Styrene: Highly Active, Versatile, and Photoresponsive Systems. Macromolecules 2008;41:7359-7367.

[273] Asandei AD, Adebolu OI, Simpson CP. Mild-Temperature Mn-2(CO)(10)Photomediated Controlled Radical Polymerization of Vinylidene Fluoride and Synthesis of Well-Defined Poly(vinylidene fluoride) Block Copolymers. J Am Chem Soc 2012;134:6080-6083.

[274] Simpson CP, Adebolu OI, Kim JS, Vasu V, Asandei AD. Metal and Ligand Effects of Photoactive Transition Metal Carbonyls in the Iodine Degenerative Transfer Controlled Radical Polymerization and Block Copolymerization of Vinylidene Fluoride. Macromolecules 2015;48:6404-6420.

[275] Asandei AD, Adebolu OI, Simpson CP, Kim JS. Visible-Light Hypervalent Iodide Carboxylate Photo(trifluoro)methylations and Controlled Radical Polymerization of Fluorinated Alkenes. Angew Chem Int Ed 2013;52:10027-10030.

[276] Cernoch P, Petrova S, Cernochova Z, Kim JS, Simpson CP, Asandei AD. Mn-2(CO)(10)photomediated synthesis of poly(vinylidene fluoride)-b-poly(styrene sulfonate). Eur Polym J 2015;68:460-470. 
[277] Wolpers A, Vana P. UV Light as External Switch and Boost of Molar-Mass Control in Iodine-Mediated Polymerization. Macromolecules 2014;47:954-963.

[278] Ohtsuki A, Goto A, Kaji H. Visible-Light-Induced Reversible Complexation Mediated Living Radical Polymerization of Methacrylates with Organic Catalysts. Macromolecules 2013;46:96-102.

[279] Ohtsuki A, Lei L, Tanishima M, Goto A, Kaji H. Photocontrolled Organocatalyzed Living Radical Polymerization Feasible over a Wide Range of Wavelengths. J Am Chem Soc 2015;137:5610-5617.

[280] Yamago S, Ukai Y, Matsumoto A, Nakamura Y. Organotellurium-Mediated Controlled/Living Radical Polymerization Initiated by Direct C-Te Bond Photolysis. J Am Chem Soc 2009;131:2100-2101.

[281] Nakamura Y, Yu M, Ukai Y, Yamago S. Organotellurium-Mediated Radical Polymerization under Photo Irradiation. In: Matyjaszewski K, Sumerlin BS, Tsarevsky NV, Chiefari J, editors. Controlled Radical Polymerization: Mechanisms. Washington DC: American Chemical Society, 2015. p. 295-309.

[282] Nakamura Y, Yamago S. Organotellurium-mediated living radical polymerization under photoirradiation by a low-intensity light-emitting diode. Beilstein J Org Chem 2013;9:1607-1612.

[283] Benedikt S, Moszner N, Liska R. Benzoyl Phenyltelluride as Highly Reactive VisibleLight TERP-Reagent for Controlled Radical Polymerization. Macromolecules 2014;47:5526-5531.

[284] Ogawa KA, Goetz AE, Boydston AJ. Metal-Free Ring-Opening Metathesis Polymerization. J Am Chem Soc 2015;137:1400-1403.

[285] Gesmundo NJ, Nicewicz DA. Cyclization-endoperoxidation cascade reactions of dienes mediated by a pyrylium photoredox catalyst. Beilstein J Org Chem 2014;10:1272-1281.

[286] Riener M, Nicewicz DA. Synthesis of cyclobutane lignans via an organic single electron oxidant-electron relay system. Chem Sci 2013;4:2625-2629.

[287] Martiny M, Steckhan E, Esch T. Cycloaddition Reactions Initiated by Photochemically Excited Pyrylium Salts. Chem Ber 1993;126:1671-1682.

[288] Goetz AE, Boydston AJ. Metal-Free Preparation of Linear and Cross-Linked Polydicyclopentadiene. J Am Chem Soc 2015;137:7572-7575.

[289] Kolb HC, Finn MG, Sharpless KB. Click Chemistry: Diverse Chemical Function from a Few Good Reactions. Angew Chem Int Ed 2001;40:2004-2021. 
[290] Tornøe CW, Christensen C, Meldal M. Peptidotriazoles on Solid Phase: 1,2,3-Triazoles by Regiospecific Copper(I)-Catalyzed 1,3-Dipolar Cycloadditions of Terminal Alkynes to Azides. J Org Chem 2002;67:3057-3064.

[291] Rostovtsev VV, Green LG, Fokin VV, Sharpless KB. A Stepwise Huisgen Cycloaddition Process: Copper(I)-Catalyzed Regioselective "Ligation" of Azides and Terminal Alkynes. Angew Chem Int Ed 2002;41:2596-2599.

[292] Gao H, Matyjaszewski K. Synthesis of Star Polymers by a Combination of ATRP and the “Click” Coupling Method. Macromolecules 2006;39:4960-4965.

[293] Golas PL, Tsarevsky NV, Sumerlin BS, Matyjaszewski K. Catalyst Performance in “Click” Coupling Reactions of Polymers Prepared by ATRP: Ligand and Metal Effects. Macromolecules 2006;39:6451-6457.

[294] Sumerlin BS, Tsarevsky NV, Louche G, Lee RY, Matyjaszewski K. Highly Efficient "Click" Functionalization of Poly(3-azidopropyl methacrylate) Prepared by ATRP. Macromolecules 2005;38:7540-7545.

[295] Tsarevsky NV, Sumerlin BS, Matyjaszewski K. Step-Growth "Click" Coupling of Telechelic Polymers Prepared by Atom Transfer Radical Polymerization. Macromolecules 2005;38:3558-3561.

[296] Demirci G, Tasdelen MA. Synthesis and characterization of graft copolymers by photoinduced CuAAC click chemistry. Eur Polym J 2015;66:282-289.

[297] Tinmaz HB, Arslan I, Tasdelen MA. Star polymers by photoinduced copper-catalyzed azide-alkyne cycloaddition click chemistry. J Polym Sci Part A Polym Chem 2015;53:1687-1695.

[298] Doganci E, Tasdelen MA, Yilmaz F. Synthesis of Miktoarm Star-Shaped Polymers with POSS Core via a Combination of CuAAC Click Chemistry, ATRP, and ROP Techniques. Macromol Chem Phys 2015;216:1823-1830.

[299] Johnson JA, Finn MG, Koberstein JT, Turro NJ. Synthesis of photocleavable linear macromonomers by ATRP and star macromonomers by a tandem ATRP-click reaction: Precursors to photodegradable model networks. Macromolecules 2007;40:3589-3598.

[300] Geng J, Lindqvist J, Mantovani G, Haddleton DM. Simultaneous copper(I)-catalyzed azide-alkyne cycloaddition (CuAAC) and living radical polymerization. Angew Chem Int Ed 2008;47:4180-4183. 
[301] Lammens M, Fournier D, Fijten MWM, Hoogenboom R, Du Prez F. Star-Shaped Polyacrylates: Highly Functionalized Architectures via CuAAC Click Conjugation. Macromol Rapid Commun 2009;30:2049-2055.

[302] Fu R, Fu GD. Polymeric nanomaterials from combined click chemistry and controlled radical polymerization. Polym Chem 2011;2:465-475.

[303] Golas PL, Matyjaszewski K. Marrying click chemistry with polymerization: expanding the scope of polymeric materials. Chem Soc Rev 2010;39:1338-1354.

[304] Golas PL, Matyjaszewski K. Click chemistry and ATRP: a beneficial union for the preparation of functional materials. QSAR Comb Sci 2007;26:1116-1134.

[305] Matyjaszewski K, Nakagawa Y, Gaynor SG. Synthesis of well-defined azido and aminoendfunctionalized polystyrene by atom transfer radical polymerization. Macromol Rapid Commun 1997; 18:1057-1066.

[306] Murtezi E, Yagci Y. Simultaneous Photoinduced ATRP and CuAAC Reactions for the Synthesis of Block Copolymers. Macromol Rapid Commun 2014;35:1782-1787.

[307] Doran S, Yagci Y. Graft polymer growth using tandem photoinduced photoinitiator-free CuAAC/ATRP. Polym Chem 2015;6:946-952.

[308] Matyjaszewski K, Gaynor S, Greszta D, Mardare D, Shigemoto T. 'Living' and controlled radical polymerization. J Phys Org Chem 1995;8:306-315.

[309] Coca S, Paik Hj, Matyjaszewski K. Block Copolymers by Transformation of "Living" Ring-Opening Metathesis Polymerization into Controlled/"Living" Atom Transfer Radical Polymerization. Macromolecules 1997;30:6513-6516.

[310] Coca S, Matyjaszewski K. Block Copolymers by Transformation of "Living" Carbocationic into "Living" Radical Polymerization. Macromolecules 1997;30:28082810.

[311] Matyjaszewski K, Beers KL, Kern A, Gaynor SG. Hydrogels by atom transfer radical polymerization. I. Poly(N-vinylpyrrolidinone-g-styrene) via the macromonomer method. J Polym Sci Part A Polym Chem 1998;36:823-830.

[312] Fu C, Xu J, Kokotovic M, Boyer C. One-Pot Synthesis of Block Copolymers by Orthogonal Ring-Opening Polymerization and PET-RAFT Polymerization at Ambient Temperature. ACS Macro Lett 2016;5:444-449.

[313] Fu C, Xu J, Boyer C. Photoacid-mediated ring opening polymerization driven by visible light. Chem Commun 2016;52:7126-7129. 
[314] Huang J, Murata H, Koepsel RR, Russell AJ, Matyjaszewski K. Antibacterial Polypropylene via Surface-Initiated Atom Transfer Radical Polymerization. Biomacromolecules 2007;8:1396-1399.

[315] Park S, Cho HY, Yoon JA, Kwak Y, Srinivasan A, Hollinger JO, Paik Hj, Matyjaszewski K. Photo-Cross-Linkable Thermoresponsive Star Polymers Designed for Control of CellSurface Interactions. Biomacromolecules 2010;11:2647-2652.

[316] Xiong X, Liu W, Luan Y, Du J, Wu Z, Chen H. A versatile, fast, and efficient method of visible-light-induced surface grafting polymerization. Langmuir 2014;30:5474-5480.

[317] Poelma JE, Fors BP, Meyers GF, Kramer JW, Hawker CJ. Fabrication of complex threedimensional polymer brush nanostructures through light-mediated living radical polymerization. Angew Chem Int Ed 2013;52:6844-6848.

[318] Pester CW, Poelma JE, Narupai B, Patel SN, Su GM, Mates TE, Luo Y, Ober CK, Hawker CJ, Kramer EJ. Ambiguous anti-fouling surfaces: Facile synthesis by lightmediated radical polymerization. J Polym Sci Part A Polym Chem 2016;54:253-262.

[319] Huang Z, Feng C, Guo H, Huang X. Direct functionalization of poly(vinyl chloride) by photo-mediated ATRP without a deoxygenation procedure. Polym Chem 2016;7:30343045.

[320] Meng JQ, Li JH, Zhang YF, Ma SN. A novel controlled grafting chemistry fully regulated by light for membrane surface hydrophilization and functionalization. $\mathrm{J}$ Membrane Sci 2014;455:405-414.

[321] Discekici EH, Pester CW, Treat NJ, Lawrence J, Mattson KM, Narupai B, Toumayan EP, Luo Y, McGrath AJ, Clark PG, Read de Alaniz J, Hawker CJ. Simple Benchtop Approach to Polymer Brush Nanostructures Using Visible-Light-Mediated Metal-Free Atom Transfer Radical Polymerization. ACS Macro Lett 2016;5:258-262.

[322] Yan J, Pan X, Schmitt M, Wang Z, Bockstaller MR, Matyjaszewski K. Enhancing Initiation Efficiency in Metal-Free Surface-Initiated Atom Transfer Radical Polymerization (SI-ATRP). ACS Macro Lett 2016;5:661-665.

[323] Nakayama Y, Matsuda T. Surface Macromolecular Architectural Designs Using PhotoGraft Copolymerization Based on Photochemistry of BenzylN,N-Diethyldithiocarbamate. Macromolecules 1996;29:8622-8630.

[324] Luo N, Hutchison JB, Anseth KS, Bowman CN. Synthesis of a novel methacrylic monomer iniferter and its application in surface photografting on crosslinked polymer substrates. J Polym Sci Part A Polym Chem 2002;40:1885-1891. 
[325] de Boer B, Simon HK, Werts MPL, van der Vegte EW, Hadziioannou G. "Living” Free Radical Photopolymerization Initiated from Surface-Grafted Iniferter Monolayers. Macromolecules 2000;33:349-356.

[326] Ward JH, Bashir R, Peppas NA. Micropatterning of biomedical polymer surfaces by novel UV polymerization techniques. Jf Biomed Mater Res 2001;56:351-360.

[327] Higashi J, Nakayama Y, Marchant RE, Matsuda T. High-Spatioresolved Microarchitectural Surface Prepared by Photograft Copolymerization Using Dithiocarbamate: Surface Preparation and Cellular Responses. Langmuir 1999;15:20802088.

[328] Luo N, Metters AT, Hutchison JB, Bowman CN, Anseth KS. A Methacrylated Photoiniferter as a Chemical Basis for Microlithography: Micropatterning Based on Photografting Polymerization. Macromolecules 2003;36:6739-6745.

[329] Lee HJ, Nakayama Y, Matsuda T. Spatio-Resolved, Macromolecular Architectural Surface: Highly Branched Graft Polymer via Photochemically Driven Quasiliving Polymerization Technique. Macromolecules 1999;32:6989-6995.

[330] Nakayama Y, Sudo M, Uchida K, Matsuda T. Spatio-Resolved Hyperbranched Graft Polymerized Surfaces by Iniferter-Based Photograft Copolymerization. Langmuir 2002;18:2601-2606.

[331] Heeb R, Bielecki RM, Lee S, Spencer ND. Room-Temperature, Aqueous-Phase Fabrication of Poly(methacrylic acid) Brushes by UV-LED-Induced, Controlled Radical Polymerization with High Selectivity for Surface-Bound Species. Macromolecules 2009;42:9124-9132.

[332] Bai J, Qiu KY, Wei Y. Synthesis of polymer-inorganic hybrid nanoparticlesvia radical polymerization initiated by surface-immobilized photoiniferter. Polym Int 2003;52:853858.

[333] Imroz Ali AM, Mayes AG. Preparation of Polymeric Core-Shell and Multilayer Nanoparticles: Surface-Initiated Polymerization Using in Situ Synthesized Photoiniferters. Macromolecules 2010;43:837-844.

[334] Di J, Sogah DY. Intergallery Living Polymerization Using Silicate-Anchored Photoiniferter. A Versatile Preparatory Method for Exfoliated Silicate Nanocomposites. Macromolecules 2006;39:1020-1028.

[335] Rahane SB, Kilbey SM, Metters AT. Kinetics of Surface-Initiated PhotoiniferterMediated Photopolymerization. Macromolecules 2005;38:8202-8210. 
[336] Mastan E, Xi L, Zhu SP. What Limits the Chain Growth from Flat Surfaces in SurfaceInitiated ATRP: Propagation, Termination or Both? Macromol Theor Simul 2015;24:8999.

[337] Luo N, Hutchison JB, Anseth KS, Bowman CN. Surface-Initiated Photopolymerization of Poly(ethylene glycol) Methyl Ether Methacrylate on a DiethyldithiocarbamateMediated Polymer Substrate. Macromolecules 2002;35:2487-2493.

[338] Rahane SB, Metters AT, Kilbey II SM. Impact of Added Tetraethylthiuram Disulfide Deactivator on the Kinetics of Growth and Reinitiation of Poly(methyl methacrylate) Brushes Made by Surface-Initiated Photoiniferter-Mediated Photopolymerization. Macromolecules 2006;39:8987-8991.

[339] Rahane SB, Kilbey SM, Metters AT. Kinetic Modeling of Surface-Initiated Photoiniferter-Mediated Photopolymerization in Presence of Tetraethylthiuram Disulfide. Macromolecules 2008;41:9612-9618.

[340] Rahane SB, Metters AT, Kilbey SM. Modeling of reinitiation ability of polymer brushes grown by surface-initiated photoiniferter-mediated photopolymerization. J Polym Sci Part A Polym Chem 2010;48:1586-1593.

[341] Harris BP, Metters AT. Generation and Characterization of Photopolymerized Polymer Brush Gradients. Macromolecules 2006;39:2764-2772.

[342] Tsuji S, Kawaguchi H. Temperature-Sensitive Hairy Particles Prepared by Living Radical Graft Polymerization. Langmuir 2004;20:2449-2455.

[343] Kawaguchi H, Isono Y, Tsuji S. Hairy particles prepared by living radical graftpolymerization. Macromol Symp. 2002;179:75-88.

[344] Rahane SB, Floyd JA, Metters AT, Kilbey SM. Swelling Behavior of Multiresponsive Poly(methacrylic acid)-block--poly(N-isopropylacrylamide) Brushes Synthesized Using Surface-Initiated Photoiniferter-Mediated Photopolymerization. Adv Funct Mater 2008;18:1232-1240.

[345] Benetti EM, Zapotoczny S, Vancso GJ. Tunable Thermoresponsive Polymeric Platforms on Gold by “Photoiniferter”-Based Surface Grafting. Adv Mater 2007;19:268-271.

[346] Sui X, Zapotoczny S, Benetti EM, Memesa M, Hempenius MA, Vancso GJ. Grafting mixed responsive brushes of poly(N-isopropylacrylamide) and poly(methacrylic acid) from gold by selective initiation. Polym Chem 2011;2:879-884. 
[347] Wolski K, Gruszkiewicz A, Zapotoczny S. Conductive polythiophene-based brushes grafted from an ITO surface via a self-templating approach. Polym Chem 2015;6:75057513.

[348] Nakayama Y, Matsuda T. Surface Macromolecular Microarchitecture Design: Biocompatible Surfaces via Photo-Block-Graft-Copolymerization UsingN,NDiethyldithiocarbamate. Langmuir 1999;15:5560-5566.

[349] Ma J, Luan S, Song L, Jin J, Yuan S, Yan S, Yang H, Shi H, Yin J. Fabricating a Cycloolefin Polymer Immunoassay Platform with a Dual-Function Polymer Brush via a Surface-Initiated Photoiniferter-Mediated Polymerization Strategy. ACS Appl Mater Interfaces 2014;6:1971-1978.

[350] Ma J, Luan SF, Jin J, Song LJ, Yuan SS, Zheng WL, Yin JH. Surface modification of cycloolefin polymer via surface-initiated photoiniferter-mediated polymerization for suppressing bioadhesion. RSC Adv 2014;4:23528-23534.

[351] Krause JE, Brault ND, Li Y, Xue H, Zhou Y, Jiang S. Photoiniferter-mediated polymerization of zwitterionic carboxybetaine monomers for low-fouling and functionalizable surface coatings. Macromolecules 2011;44:9213-9220.

[352] Liu Q, Singh A, Liu L. Amino acid-based zwitterionic poly(serine methacrylate) as an antifouling material. Biomacromolecules 2013;14:226-231.

[353] Huang CJ, Li Y, Krause JB, Brault ND, Jiang S. Internal architecture of zwitterionic polymer brushes regulates nonfouling properties. Macromol Rapid Commun 2012;33:1003-1007.

[354] Huang CJ, Li Y, Jiang S. Zwitterionic polymer-based platform with two-layer architecture for ultra low fouling and high protein loading. Anal Chem 2012;84:34403445.

[355] Huang CJ, Brault ND, Li Y, Yu Q, Jiang S. Controlled hierarchical architecture in surface-initiated zwitterionic polymer brushes with structurally regulated functionalities. Adv Mater 2012;24:1834-1837.

[356] Li W, Liu Q, Liu L. Antifouling gold surfaces grafted with aspartic acid and glutamic acid based zwitterionic polymer brushes. Langmuir 2014;30:12619-12626.

[357] Liu Q, Li W, Singh A, Cheng G, Liu L. Two amino acid-based superlow fouling polymers: poly(lysine methacrylamide) and poly(ornithine methacrylamide). Acta Biomater 2014;10:2956-2964. 
[358] Matsuda T, Ohya S. Photoiniferter-based thermoresponsive graft architecture with albumin covalently fixed at growing graft chain end. Langmuir 2005;21:9660-9665.

[359] Sellergren B, Rückert B, Hall AJ. Layer-by-Layer Grafting of Molecularly Imprinted Polymers via Iniferter Modified Supports. Adv Mater 2002;14:1204-1208.

[360] Singh M, Tarannum N, Kumar A. Selective recognition of fenbufen by surface-imprinted silica with iniferter technique. J Porous Mat 2014;21:677-684.

[361] Lakshmi D, Bossi A, Whitcombe MJ, Chianella I, Fowler SA, Subrahmanyam S, Piletska EV, Piletsky SA. Electrochemical Sensor for Catechol and Dopamine Based on a Catalytic Molecularly Imprinted Polymer-Conducting Polymer Hybrid Recognition Element. Anal Chem 2009;81:3576-3584.

[362] Sebra RP, Anseth KS, Bowman CN. Integrated surface modification of fully polymeric microfluidic devices using living radical photopolymerization chemistry. J Polym Sci Part A Polym Chem 2006;44:1404-1413.

[363] Yu HY, Li W, Zhou J, Gu JS, Huang L, Tang ZQ, Wei XW. Thermo- and pH-responsive polypropylene microporous membrane prepared by the photoinduced RAFT-mediated graft copolymerization. J Membrane Sci 2009;343:82-89.

[364] Yang S, Gu JS, Yu HY, Zhou J, Li SF, Wu XM, Wang L. Polypropylene membrane surface modification by RAFT grafting polymerization and $\mathrm{TiO} 2$ photocatalysts immobilization for phenol decomposition in a photocatalytic membrane reactor. Sep Purif Technol 2011;83:157-165.

[365] Hu B, Wang L, Wu XM, Yang S, Gu JS, Yu HY. Low protein fouling polypropylene membrane prepared by photoinduced reversible addition-fragmentation chain transfer polymerization. J Appl Polym Sci 2012;123:3668-3674.

[366] Halhalli MR, Aureliano CSA, Schillinger E, Sulitzky C, Titirici MM, Sellergren B. An improved grafting technique for producing imprinted thin film composite beads. Polym Chem 2012;3:1033-1042.

[367] Telitel S, Telitel S, Bosson J, Spangenberg A, Lalevee J, Morlet-Savary F, Clement JL, Guillaneuf Y, Gigmes D, Soppera O. Nitroxide Mediated Photopolymerization: A Versatile Tool for the Fabrication of Complex Multilayer Polyfunctional Copolymer Nanostructures. Adv Mater Interfaces 2014;1:1400067/1-5.

[368] Telitel S, Telitel S, Bosson J, Lalevée J, Clément JL, Godfroy M, Fillaut JL, Akdas-Kilig H, Guillaneuf Y, Gigmes D, Soppera O. UV-Induced Micropatterning of Complex 
Functional Surfaces by Photopolymerization Controlled by Alkoxyamines. Langmuir 2015;31:10026-10036.

[369] Telitel S, Amamoto Y, Poly J, Morlet-Savary F, Soppera O, Lalevee J, Matyjaszewski K. Introduction of self-healing properties into covalent polymer networks via the photodissociation of alkoxyamine junctions. Polym Chem 2014;5:921-930. 
Figure 1. General presentation of photoinitiated polymerization.

Figure 2. Mechanism of unimolecular (upper) or bimolecular (lower) photoinitiated systems.

Figure 3. Commonly used photoredox catalysts.

Figure 4. General mechanism for oxidative photoredox reactions.

Figure 5. Proposed mechanisms for photomediated ATRP using unimolecular and bimolecular photoinitiated systems (PI: photoinitiator; PS: photosensitizer and R$\mathrm{H}$ : hydrogen donor).

Figure 6. Postulated mechanism of photomediated ATRP using a heterogeneous photocatalyst.

Figure 7. Sun-light mediated ATRP using $\mathrm{Mn}_{2}(\mathrm{CO})_{10} / \mathrm{Cu}(\mathrm{II}) \mathrm{Br}_{2} /$ alkyl halide system. [61], Copyright 2014. Reproduced with permission from the Royal Society of Chemistry.

Figure 8. General reaction scheme for a photoATRP reaction using copper(II) as catalyst.

Figure 9. Comparison of various copper and ligand concentrations: (1.h.s.) kinetic plot, (r.h.s.) evolution of molar mass and dispersity with conversion. Reactions were

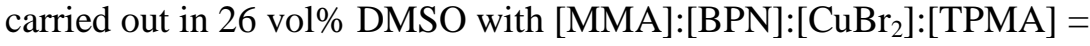
200:1:0.02:0.20; [MMA] $=6.9 \mathrm{M} ; \mathrm{T}=35^{\circ} \mathrm{C} ; \lambda<350 \mathrm{~nm}$. [74], Copyright 2015. Reproduced with permission from the Royal Society of Chemistry.

Figure 10. (A) Kinetics and (B) $M_{n}$ (solid points) and $M_{w} / M_{n}$ (open points) evolution in the polymerization of MA using different radiation sources. Conditions:

[MA]:[EBiB]:[CuBr 2$]:\left[\mathrm{TMPA}^{*}\right]=300: 1: 0.03: 0.135$ in $50 \mathrm{vol} \% \mathrm{DMF}$ at room temperature. [23], Copyright 2012. Reproduced with permission from the American Chemical Society.

Figure 11. Evidence of temporal control via consecutive light (white area) and dark (shaded area) exposure. $[\mathrm{M}]:[\mathrm{EBiB}]:\left[\mathrm{CuBr}_{2}\right]:\left[\mathrm{Me}_{6} \mathrm{TREN}\right]=50: 1: 0.02: 0.12$ in DMSO (50\%, v/v) solvent. [25], Copyright 2014. Reproduced with permission from the American Chemical Society.

Figure 12. Photoinduced ATRP of OEOMA ${ }_{500}$ in water with ppm of $\mathrm{Cu}$ with or without added salt. (A) Semilogarithmic kinetic plots (B) evolution of $M_{\mathrm{n}}$ (filled symbols) and (C) $M_{\mathrm{w}} / M_{\mathrm{n}}$ (open symbols) with conversion, conditions:

$\left[\mathrm{OEOMA}_{500}\right]_{0} /\left[\mathrm{PEO}_{2000} \mathrm{BPA}\right]_{0} /\left[\mathrm{CuBr}_{2}\right]_{0} /[\mathrm{TPMA}]_{0}=450 / 1 / \mathrm{x} / 4 \mathrm{x}$ in $90 \%$ water with 
$392 \mathrm{~nm}$ at $0.9 \mathrm{~mW} / \mathrm{cm}^{2}$ at room temperature. [87], Copyright 2015. Reproduced with permission from the American Chemical Society.

Figure 13. (a) Kinetics and (b) evolution of $M_{\mathrm{n}}$ (filled symbols) and $M_{\mathrm{w}} / M_{\mathrm{n}}$ (opened symbols) with conversion for photoATRP of MA in DMSO under the conditions:

$[\mathrm{MA}]_{0}:[\mathrm{EBiB}]_{0}:\left[\mathrm{CuBr}_{2}\right]_{0}:\left[\mathrm{Me}_{6} \mathrm{TREN}_{0}=300: 1: 0.03: 0.03-0.18,[\mathrm{MA}]=7.4 \mathrm{M}\right.$, irradiated by $392 \mathrm{~nm}$ light $\left(0.9 \mathrm{~mW} / \mathrm{cm}^{2}\right)$ at $25^{\circ} \mathrm{C}$. [66], Copyright 2014.

Reproduced with permission from the American Chemical Society.

Figure 14. Structures of selected iron catalysts in photoATRP.

Figure 15. Photoreduction of Fe(III) to Fe(II), confirmed by UV-vis-NIR spectroscopic studies of $3 \mathrm{mM} \mathrm{FeBr}_{3}$ in $\mathrm{MeCN}$ in the presence of 100 equiv of MMA at rt with irradiation. [100], Copyright 2015. Reproduced with permission from the American Chemical Society.

Figure 16. Structures of selected Ir catalysts in photoATRP.

Figure 17. Structures of selected Ru catalysts examined for photoATRP.

Figure 18. Structures of metal-free catalysts in photoATRP.

Figure 19. PTZ-catalyzed photoATRP of benzyl methacrylate. (a) Conversion during a series of "on-off" cycles of the polymerization to light. (b) Evolution of $M_{\mathrm{n}}$ (filled symbols) and $M_{\mathrm{w}} / M_{\mathrm{n}}$ (opened symbols) with conversion. (c) Semilogarithmic kinetic plots of polymerization with irradiation. [26], Copyright 2014. Reproduced with permission from the American Chemical Society.

Figure 20. (a) Semilogarithmic kinetic plots of polymerization under conditions $[\mathrm{MMA}]_{0} /[\mathrm{EBPA}]_{0} /[\mathrm{FL}]_{0} /[\mathrm{TEA}]_{0}=200 / 1 / \mathrm{x} / \mathrm{y}(\mathrm{x}=0.1, \mathrm{y}=3$, square; $\mathrm{x}=0.2, \mathrm{y}=6$, circle; $\mathrm{x}=0.3, \mathrm{y}=9$, triangle), $\mathrm{V}_{\mathrm{MMA}}=1.0 \mathrm{~mL}, \mathrm{~V}_{\mathrm{DMSO}}=2.0 \mathrm{~mL}$; irradiation by a blue LED at rt. (b) Evolution of $M_{\mathrm{n}}$ (filled symbols) and $M_{\mathrm{w}} / M_{\mathrm{n}}$ (opened symbols) with conversion. [133], Copyright 2016. Reproduced with permission from The Royal Society of Chemistry.

Figure 21. (a) Semilogarithmic kinetic plots of polymerization under conditions $[\mathrm{AN}]_{0} /[\mathrm{EBPA}]_{0} /[\mathrm{Ph}-\mathrm{PTZ}]_{0}=100 / 1 / 0.1$, in DMSO or DMF; irradiation by a 365 $\mathrm{nm}\left(4.9 \mathrm{~mW} / \mathrm{cm}^{2}\right)$ at rt. (b) Evolution of $M_{\mathrm{n}}$ (filled symbols) and $M_{\mathrm{w}} / M_{\mathrm{n}}$ (opened symbols) with conversion. [27], Copyright 2015. Reproduced with permission from the American Chemical Society. 
Figure 22. Structures of photoredox catalysts $f a c$-[ $\left.\operatorname{Ir}(\mathrm{ppy})_{3}\right], \mathrm{Ph}-\mathrm{PTZ}$ and a traditional Cubased ATRP catalyst $\left[\mathrm{Cu}^{\mathrm{I}}\left(\mathrm{TPMA}^{*} 3\right)\right]^{+}$. [141], Copyright 2016. Reproduced with permission from the American Chemical Society.

Figure 23. Structures of 9-phenylcarbazole and thianthrene.

Figure 24. TEM images of micellized amphiphilic poly(methyl acrylate) $)_{50}$-b-(glycidyl acrylate) ${ }_{100}$ block copolymer. Scale bar $=50 \mathrm{~nm}$. [69], Copyright 2014. Reproduced with permission from the American Chemical Society.

Figure 25. Molecular weight distributions of the intermediate block copolymer products in the synthesis of decablock copolymers from sequential monomer addition. [70], Copyright 2014. Reproduced with permission from the American Chemical Society.

Figure 26. Effect of long-wave length irradiation $(\square>320 \mathrm{~nm}$ ) irradiation intensity on polymerization of methyl acrylate mediated by DDMAT at $25^{\circ} \mathrm{C}$. [206], Copyright 2013. Reproduced with permission from the American Chemical Society.

Figure 27. Formation of star polymers by an arm-first approach, using initiator-free photoRAFT to activate chains. [209], Copyright 2015. Reproduced with permission from the American Chemical Society.

Figure 28. GPC curves for PMMA produced in a two-step process: The first step proceeded via a one-stage photoinitiated RAFT dispersion polymerization with $2 \mathrm{~g}$ of MMA. After $3 \mathrm{~h}$ irradiation, $1 \mathrm{~g}$ of MMA was added to the mixture and the reaction continued for further 3 h. [237], Copyright 2012. Reproduced with permission from the American Chemical Society.

Figure 29. (A) Characterization of POEGMA- $b$-PBzMA diblock copolymers synthesized using (i) 20, (ii) 40, and (iii) 100 ppm catalyst relative to monomer concentration and a [BzMA]/[POEGMA] ratio of 200:1; (B) TEM images of self-assembled POEGMA- $b$-PBnMA nanoparticles (Exp. i, ii, and iii). [245], Copyright 2015. Reproduced with permission from the American Chemical Society.

Figure 30. Photomediated NMP using a photosensitive alkoxyamine.

Figure 31. General mechanism of photomediated CMRP using organocobalt porphyrin as a control agent.

Figure 32. Proposed mechanism of photomediated ITP with $\mathrm{Mn}_{2}(\mathrm{CO})_{10} /$ alkyl iodide. 
Figure 33. Proposed mechanism of photomediated ITP based on reversible complexation of alkyl iodides with various organic dyes.

Figure 34. General scheme of photomediated TERP based on direct homolysis of C-Te bond.

Figure 35. Structure of monomers, initiators and catalyst used in photoinduced metal-free ROMP.

Figure 36. Synthesis of block and graft copolymers via simultaneous photoinduced ATRP and $\mathrm{CuAAC}$ reactions.

Figure 37. PhotoATRP grafting of 3-sulfopropyl methacrylate from thiol-patterned gold substrate. (A) Film thickness vs. time evolution at different light intensities in comparison with conventional ATRP. (B) Comparison of film thicknesses for various light intensities for exposure times of 20 and $30 \mathrm{~min},(\mathrm{C})$ Evolution of film thickness with $\mathrm{TiO}_{2}$ particle concentration at $1.25 \mathrm{~mW} / \mathrm{cm}^{2}$ illumination for 20 min. [60], Copyright 2013. Reproduced with permission from the American Chemical Society.

Figure 38. $\wedge \mathrm{t}(1 . h . s)$ Film thickness vs. time evolution of copper-mediated photoATRP grafting of poly(t-butyl acrylate) brushes at various copper catalyst loadings (ppm with respect to monomer). (r.h.s.) XPS depth profiling of poly(t-butyl acrylate)-bpoly(2-hydroxyethyl acrylate) brushes. A composition drift from preferentially poly(2-hydroxyethyl acrylate) at the polymer/air interface to almost pure poly(tbutyl acrylate) at the polymer silicon interface. [76], Copyright 2015. Reproduced with permission from John Wiley \& Sons Inc.

Figure 39. (A) Optical micrograph and (B) AMF image of nanoscale-inclined plane formed from polymer brushes upon light exposure through a shadow mask with optical density gradient. (C) Height along dashed line across feature as shown in (A). [317], Copyright 2013. Reproduced with permission from John Wiley \& Sons Inc.

Figure 40. (1.h.s.) Fluorescence micrograph of photoiniferter grafting by UV irradiation through the stripe-patterned projection metal mask and the neutral-density filter and subsequently stained with rose bengal, and the three-dimensional image (b) of the distribution of the fluorescence intensity in the area shown in (a). [323], Copyright 1996. Reproduced with permission from the American Chemical Society; 
(r.h.s) Schematic drawing of first generation to nth generation graft architectures.

[330], Copyright 2012. Reproduced with permission from the American Chemical Society.

Figure 41. Synthetic route to conductive, iodine-doped polythiophene brushes. [347],

Copyright 2015. Reproduced with permission from The Royal Society of Chemistry.

Figure 42. Schematic representation of fabrication micropatterned films via photomediated NMP process by combination homogeneous and local irradiations.

Figure 43. Direct laser writing with Ir-mediated photoATRP using a Nd:Yag microlaser (523 $\mathrm{nm}, 0.6 \mathrm{~ns}$ ) with a $\times 40$ microscope objective. (1.h.s.) Control of line height with laser power of $5 \mathrm{~ms}$ irradiation. (r.h.s.) Logo created with $130 \mu \mathrm{W}, 10 \mathrm{~ms}$. [126], Copyright 2015. Reproduced with permission from The Royal Society of Chemistry. 


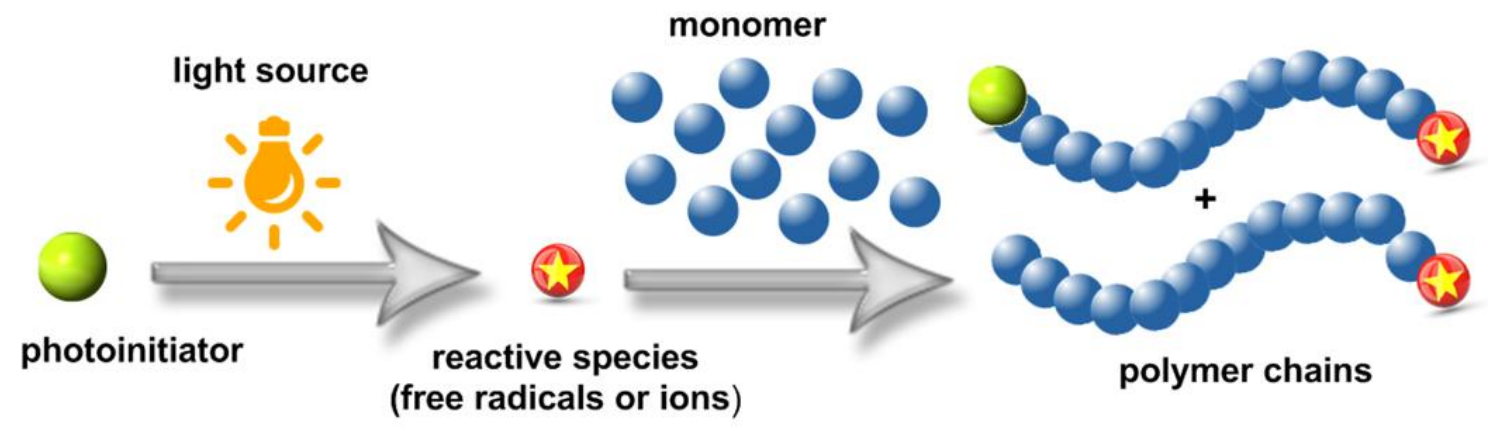

Figure 1. 


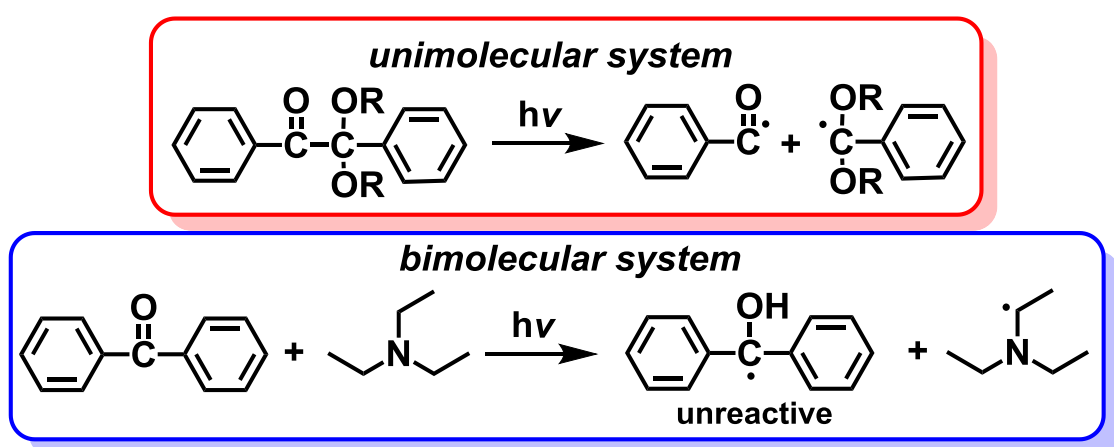

Figure 2. 


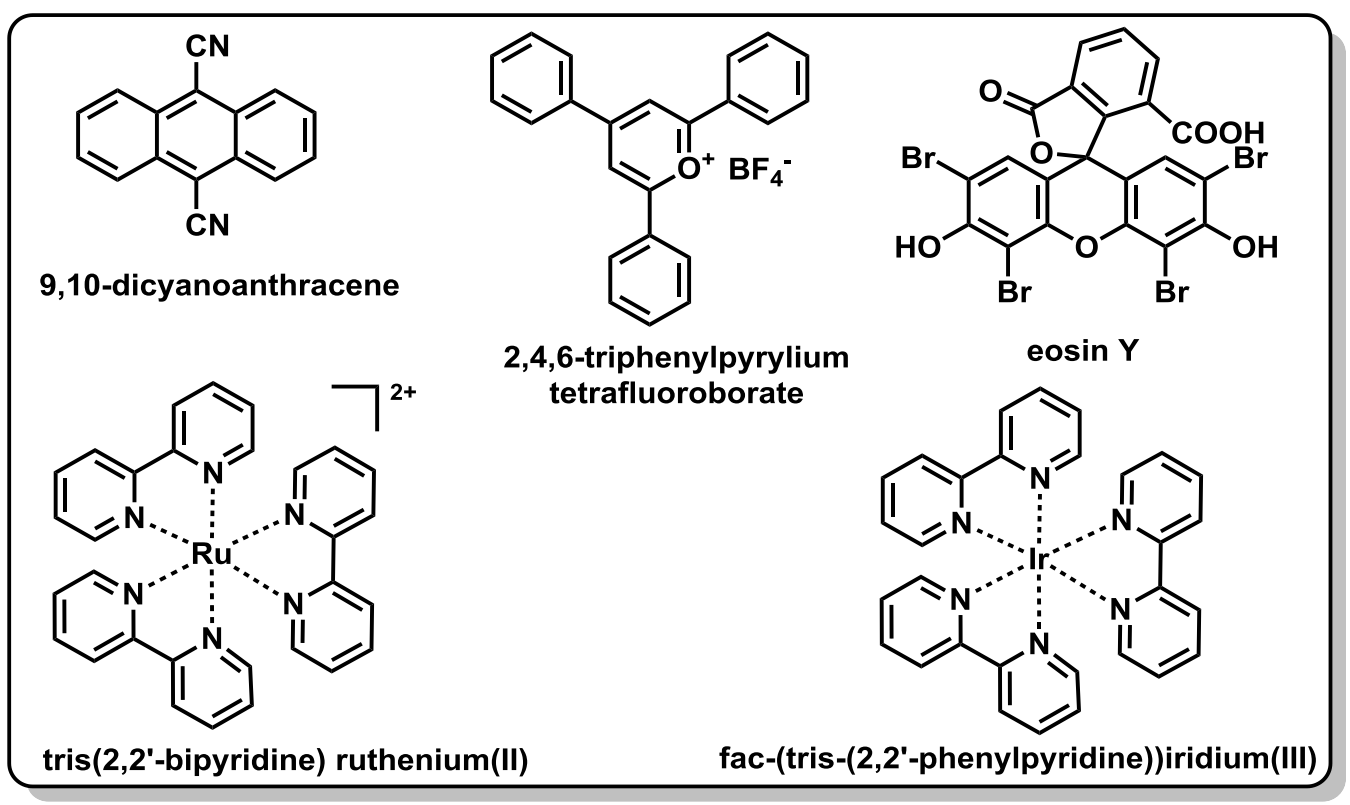

Figure 3. 


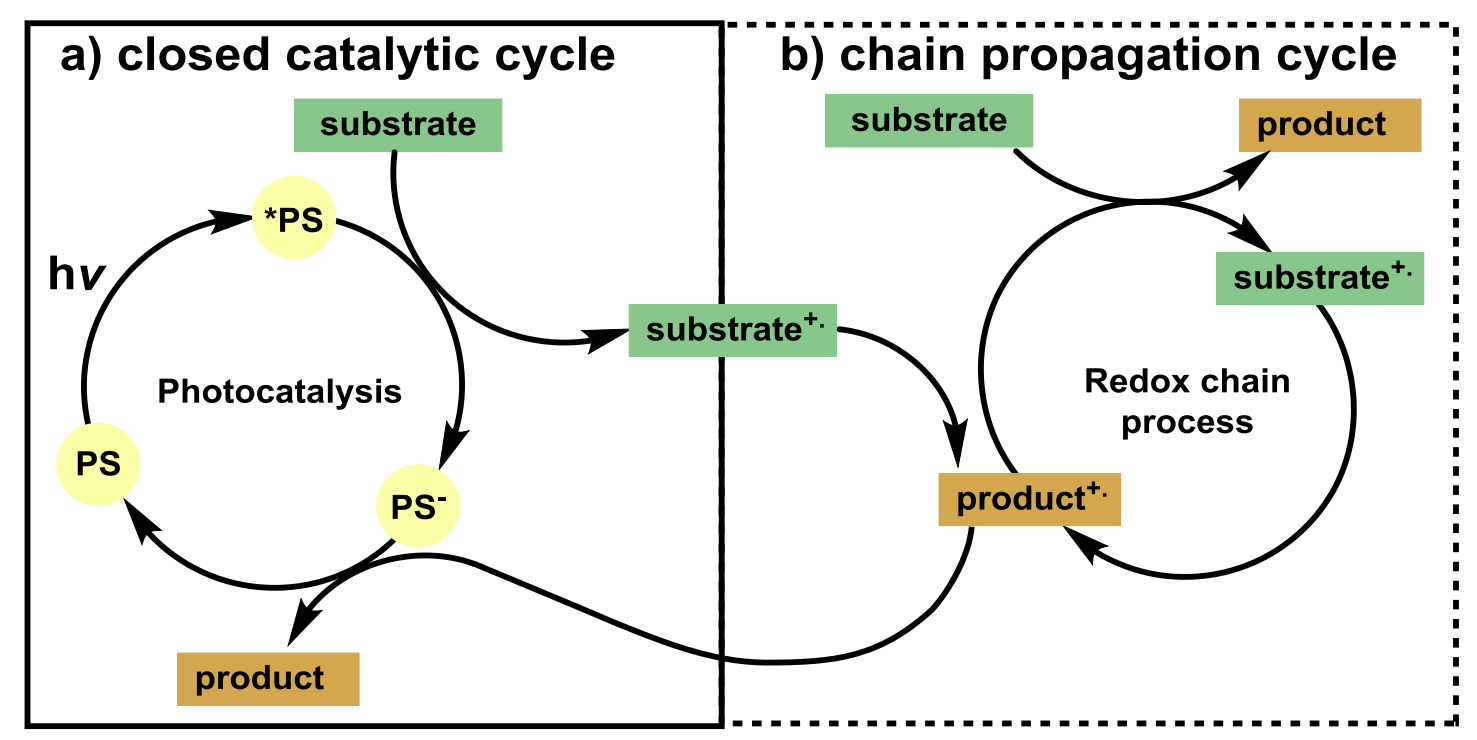

Figure 4. 


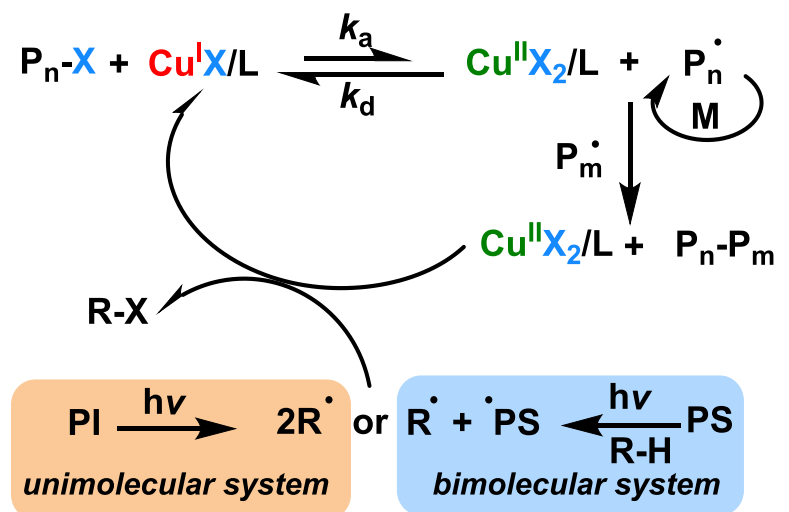

Figure 5. 


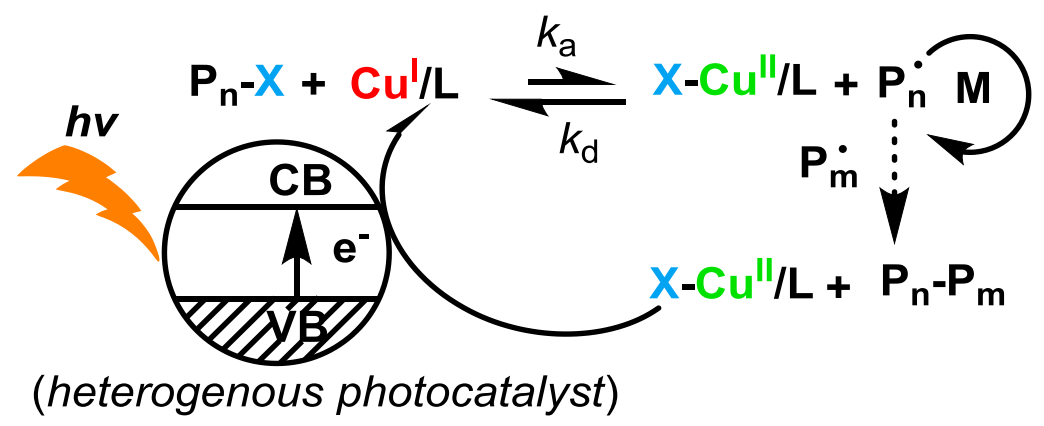

Figure 6. 


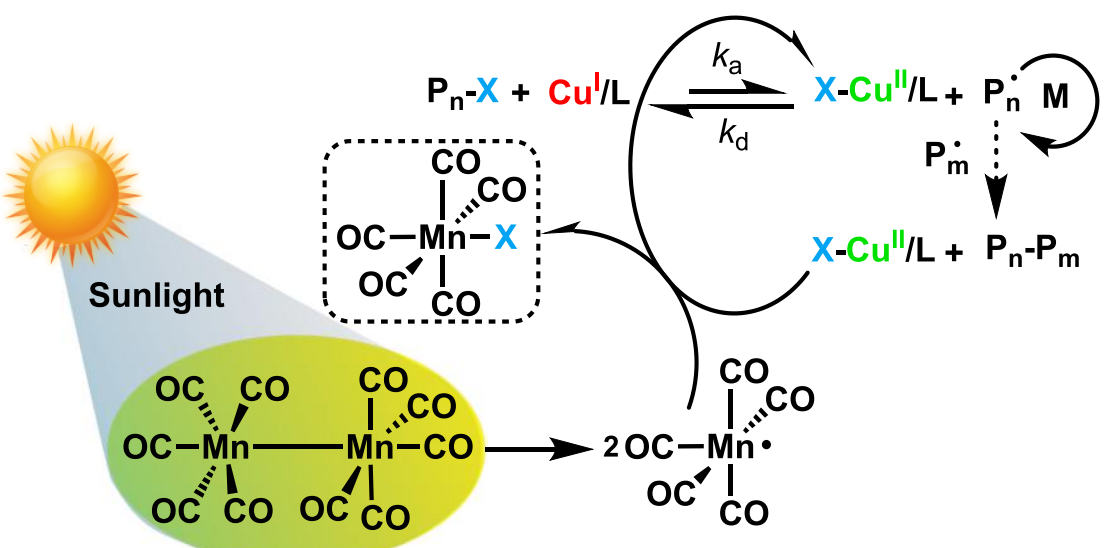

Figure 7. 


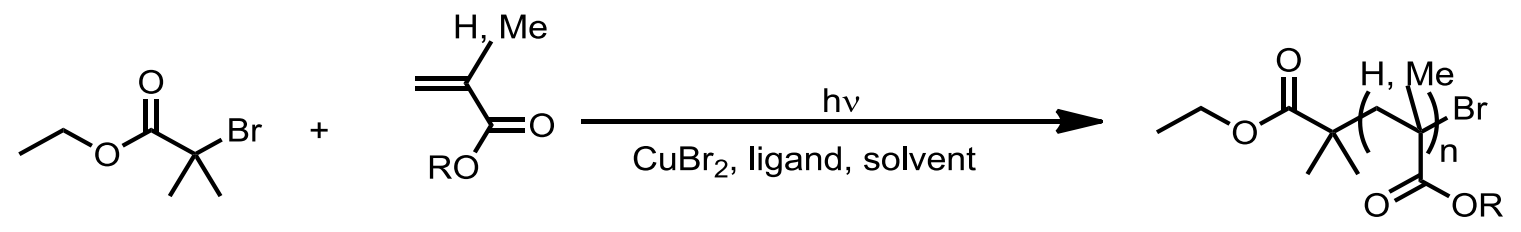

Figure 8. 

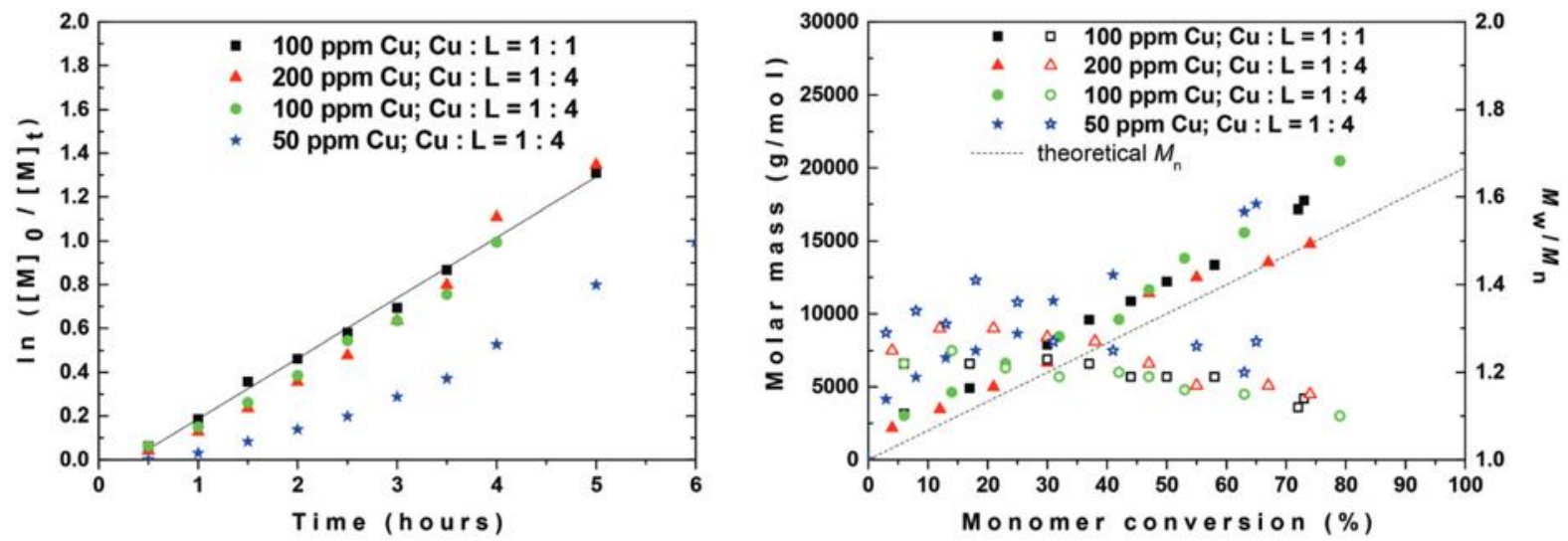

Figure 9. 

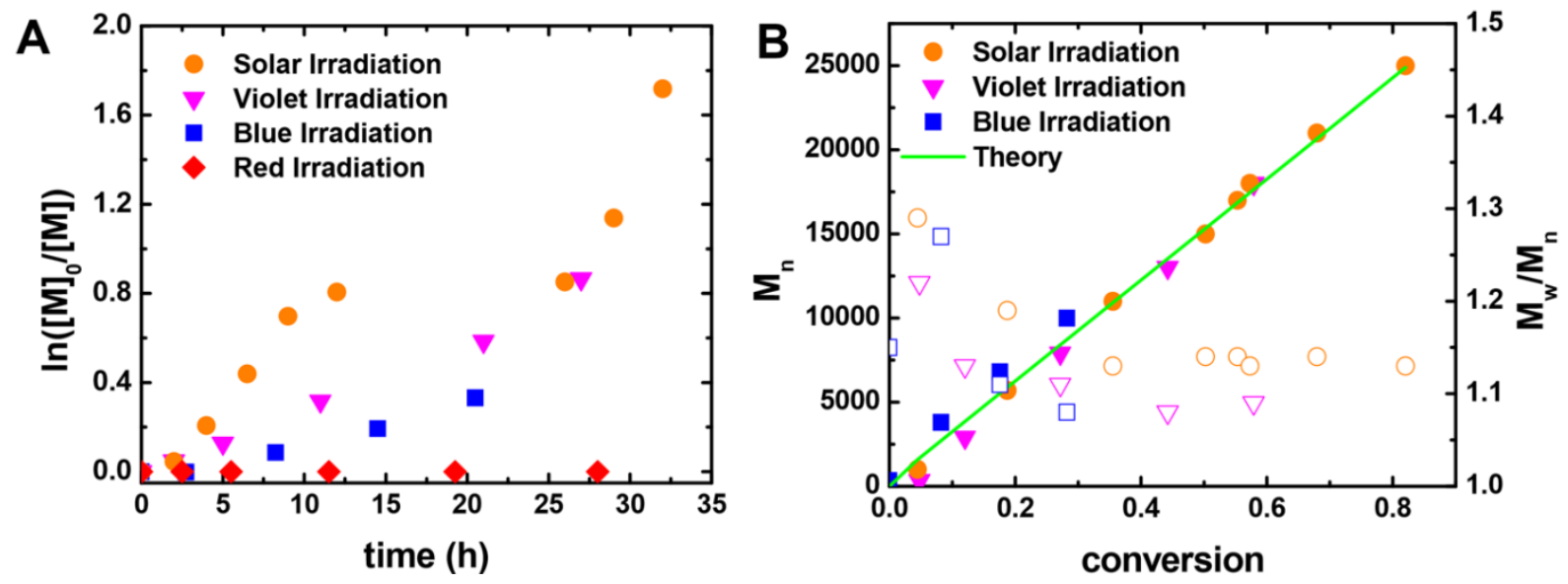

Figure 10. 


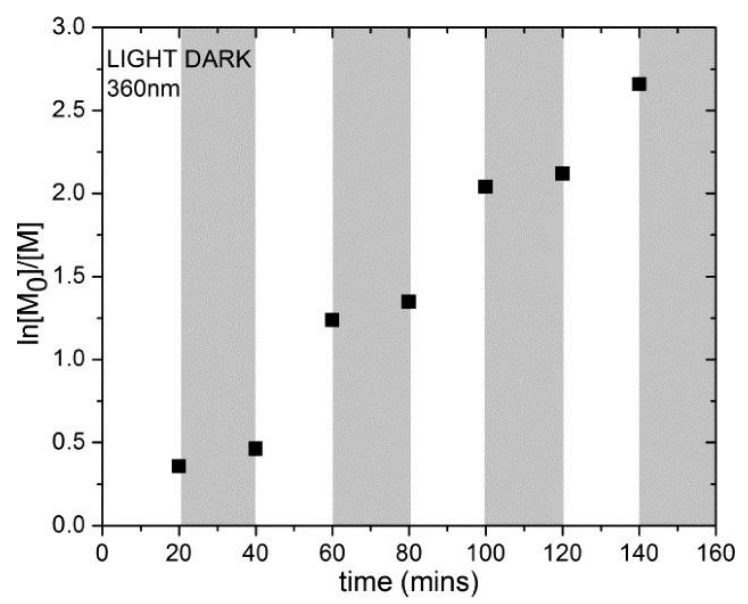

Figure 11. 
(A)

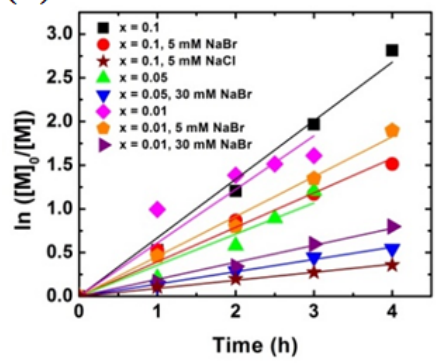

(B)

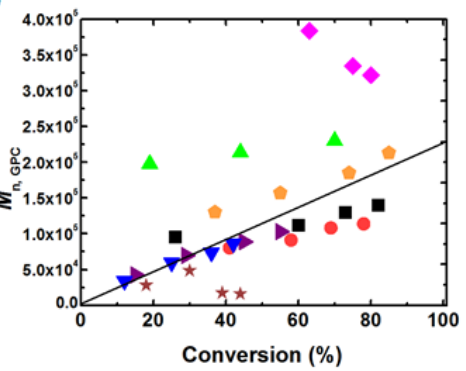

(C)

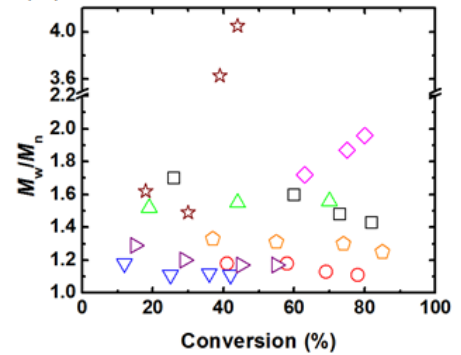

Figure 12. 


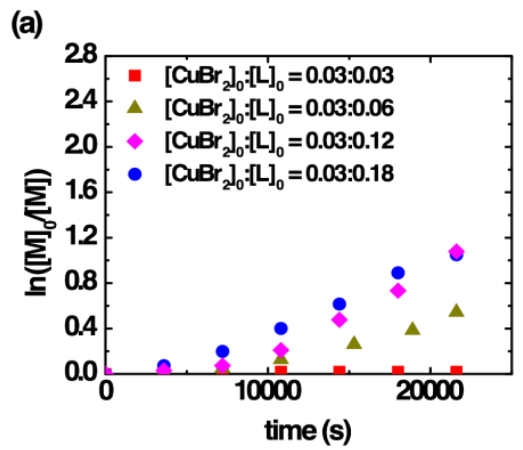

(b)

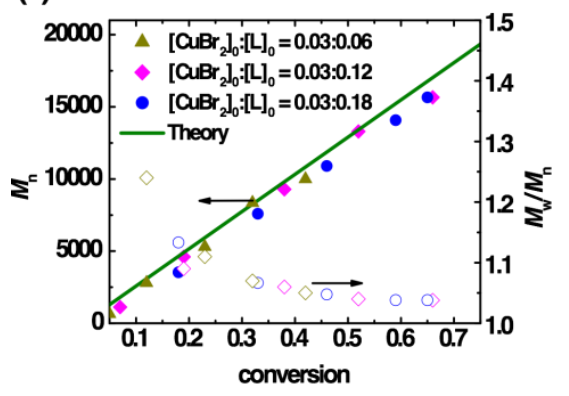

Figure 13. 

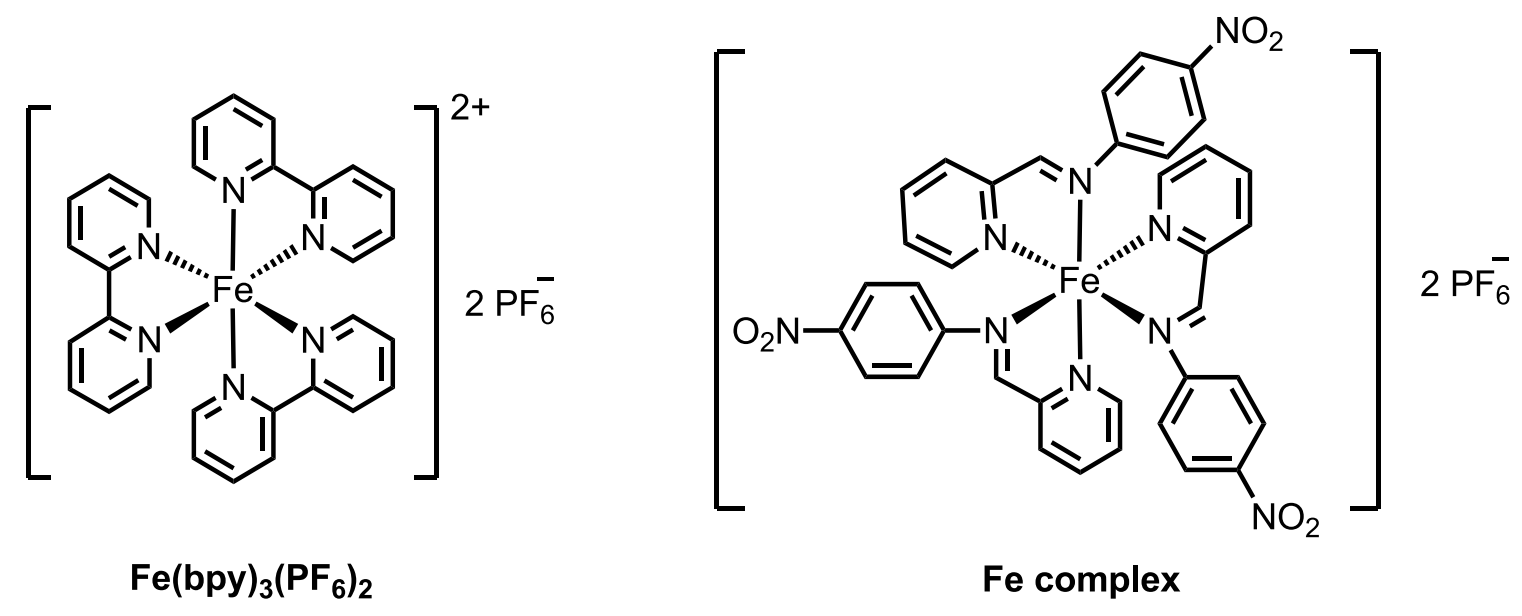

Fe complex

Figure 14. 

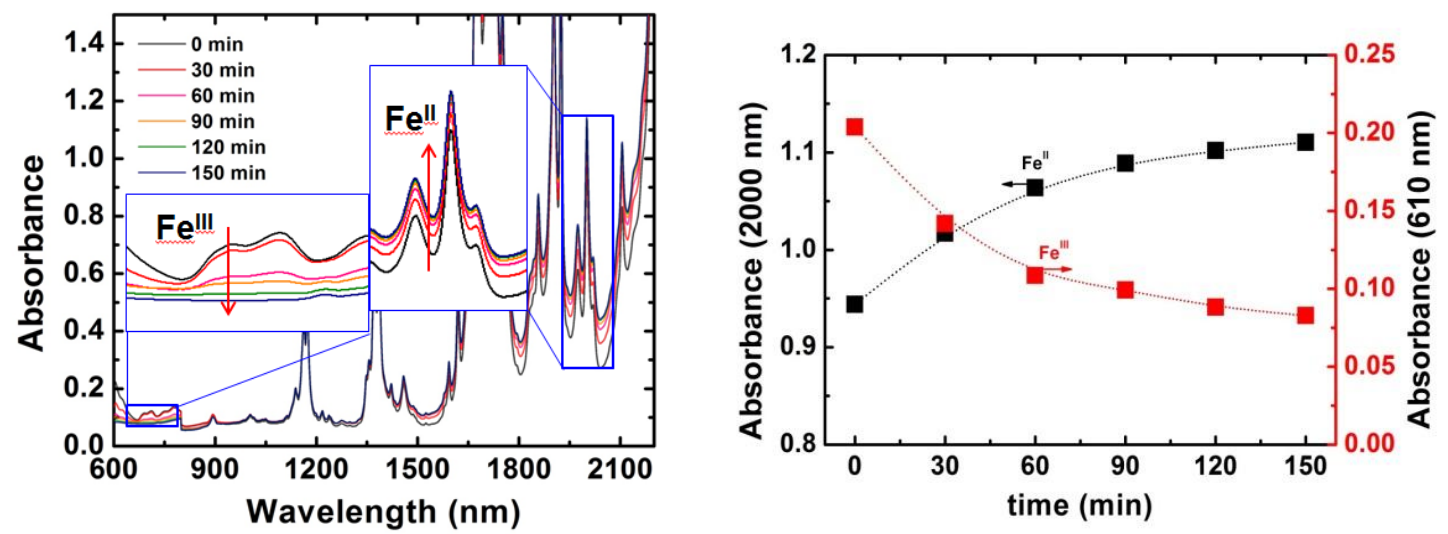

Figure 15 

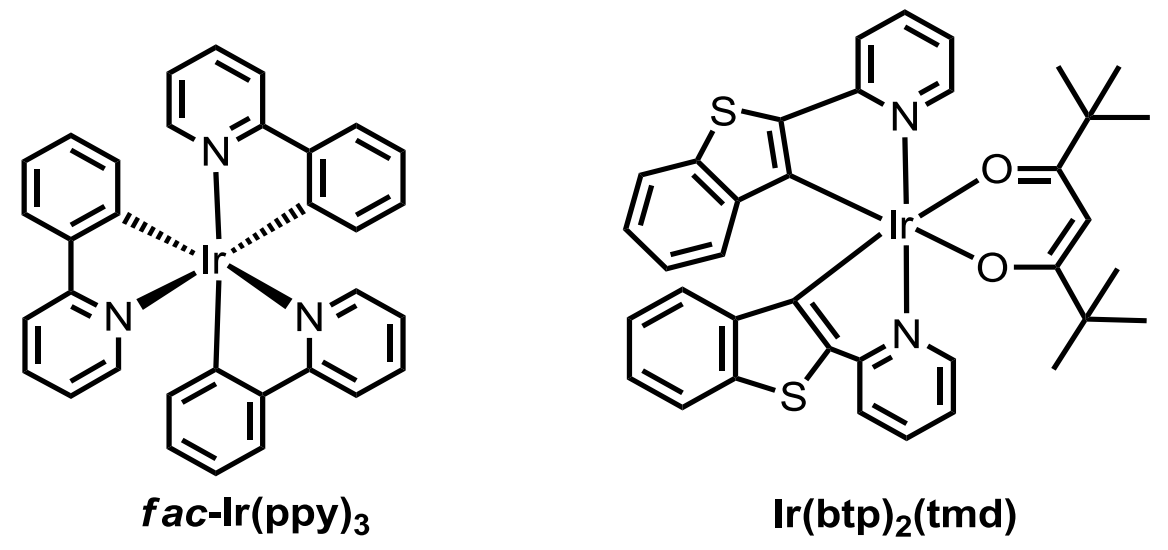

Figure 16. 


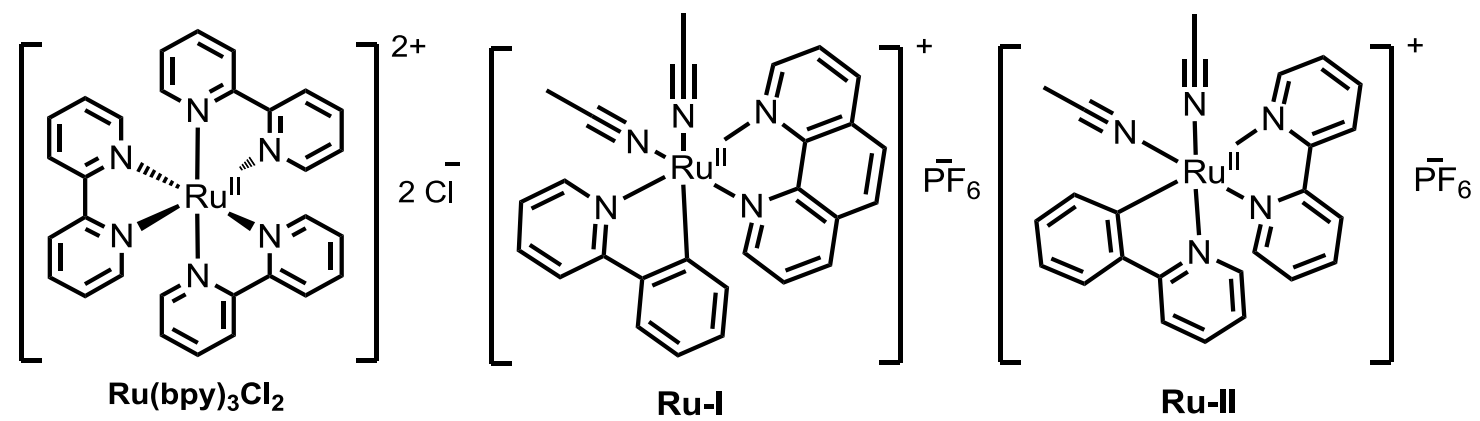

Figure 17. 


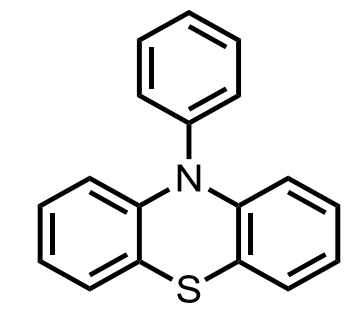

10-phenylphenothiazine

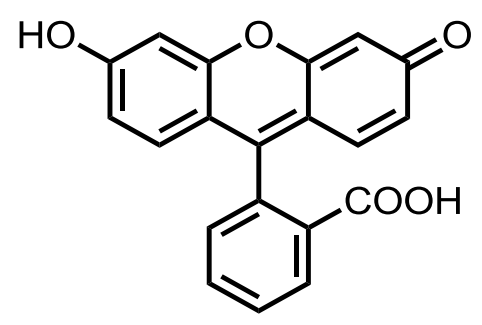

fluorescein

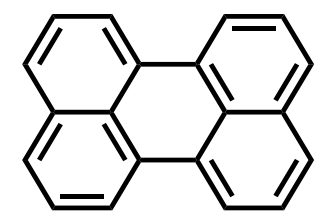

perylene

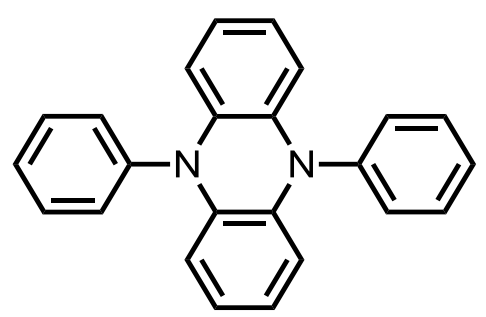

5,10-diphenyl-5,10-dihydrophenazine

Figure 18. 

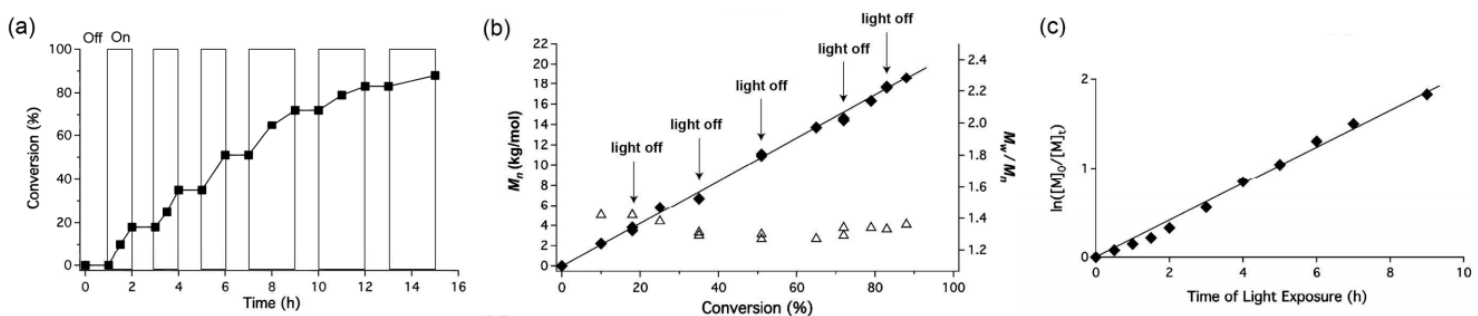

Figure 19. 

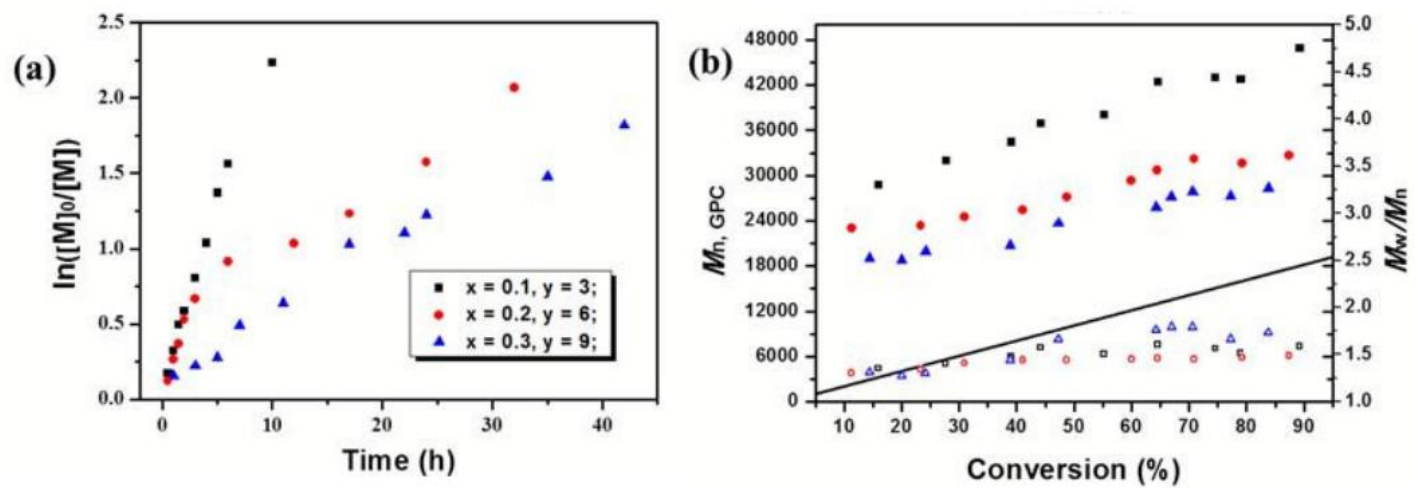

Figure 20. 

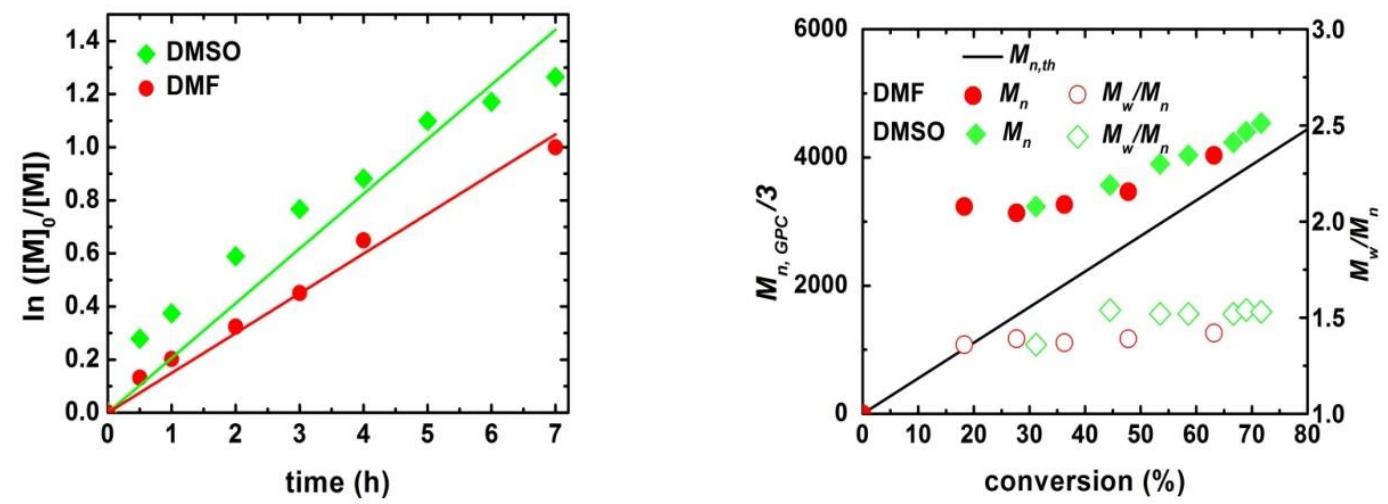

Figure 21. 

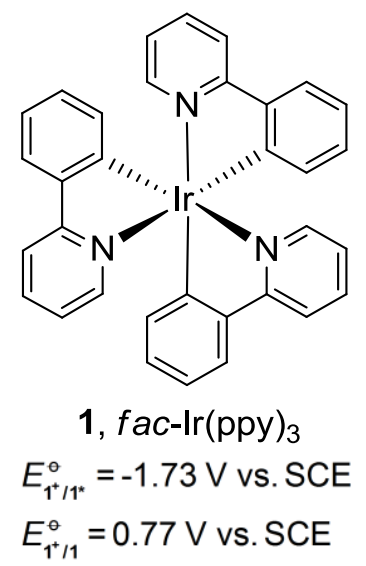

$E_{1^{\dagger} / 1}^{\ominus}=0.77 \mathrm{~V}$ vs. SCE

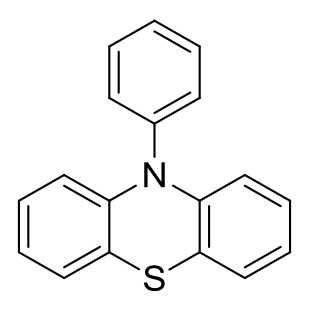

2, Ph-PTZ

$E_{2^{+/ / 2}}^{\ominus}=-2.10 \mathrm{~V}$ vs. SCE

$E_{2^{* / 1} / 2}^{\ominus}=0.68 \mathrm{Vvs}$. SCE

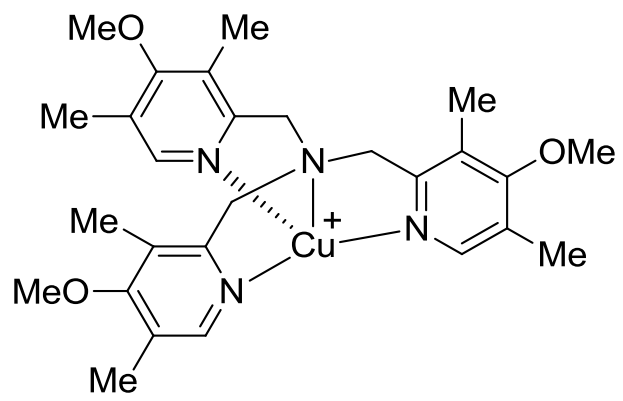

3, $\left[\mathrm{Cu}^{\prime}\left(\mathrm{TPMA}^{* 3}\right)\right]^{+}$

$E^{\ominus}=-0.18 \mathrm{~V}$ vs. SCE

Figure 22. 


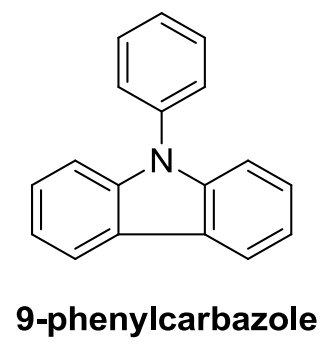

9-phenylcarbazole

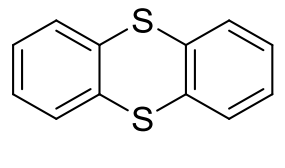

thianthrene

Figure 23. 


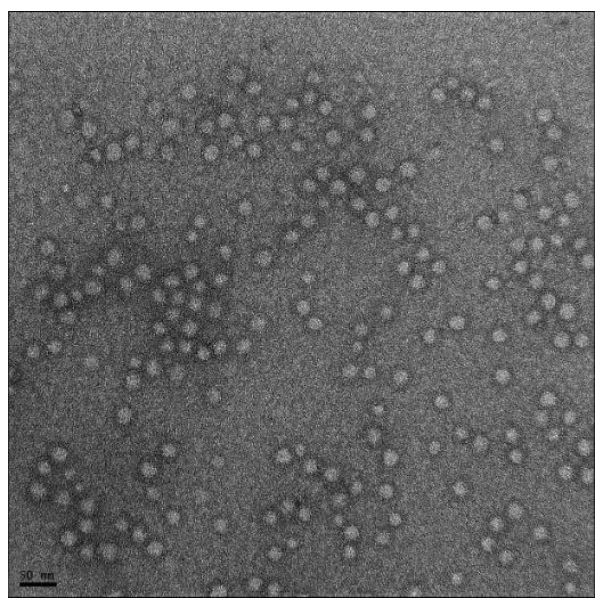

Figure 24. 


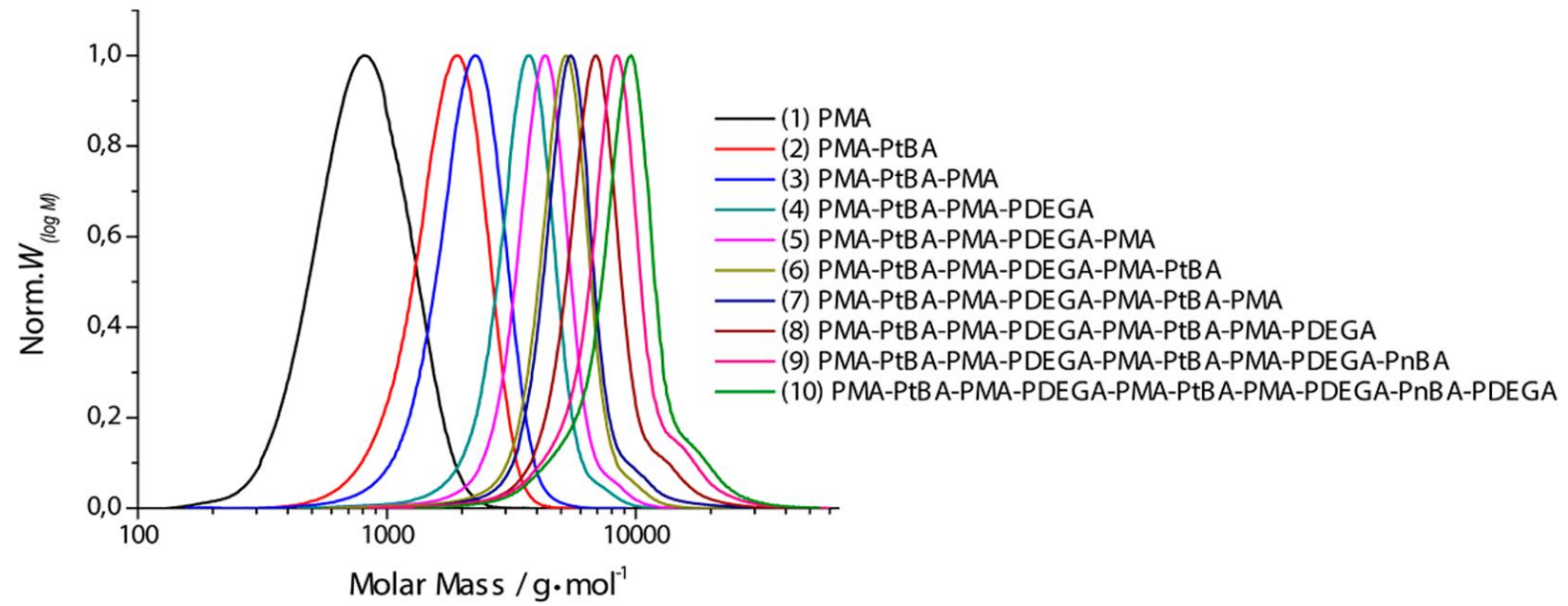

Figure 25. 


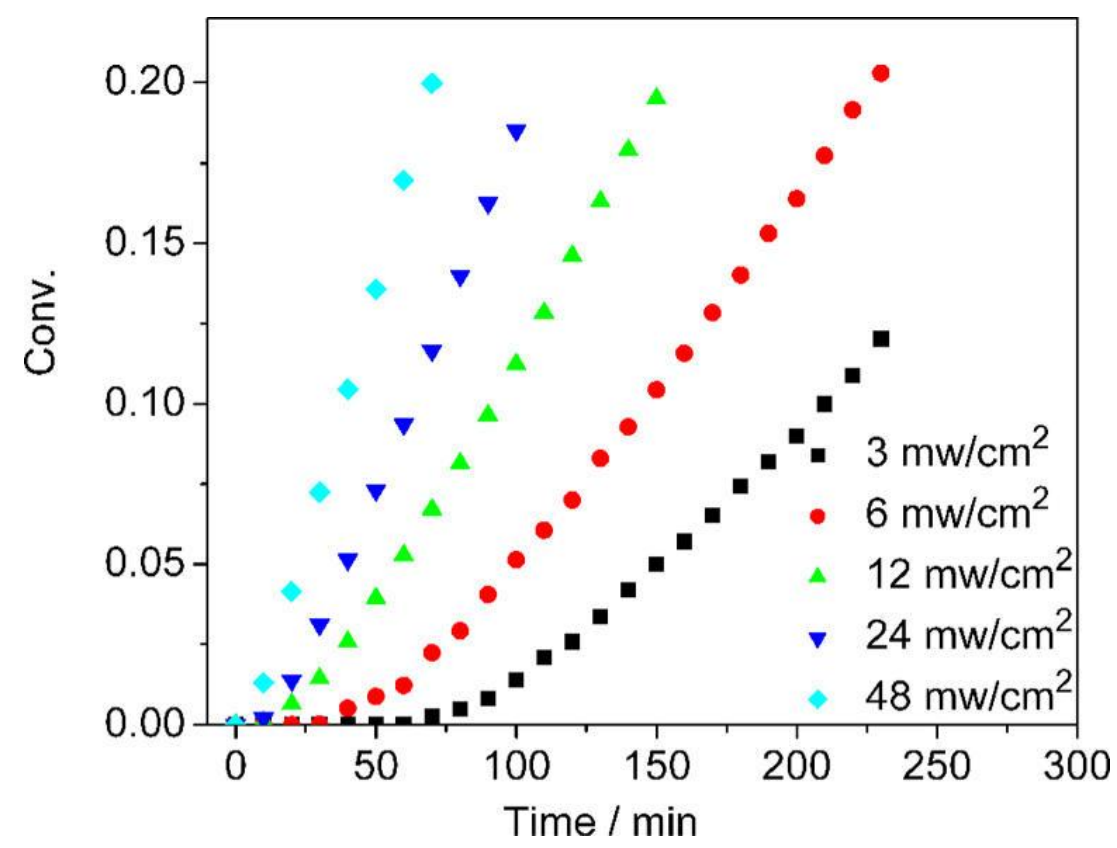

Figure 26. 


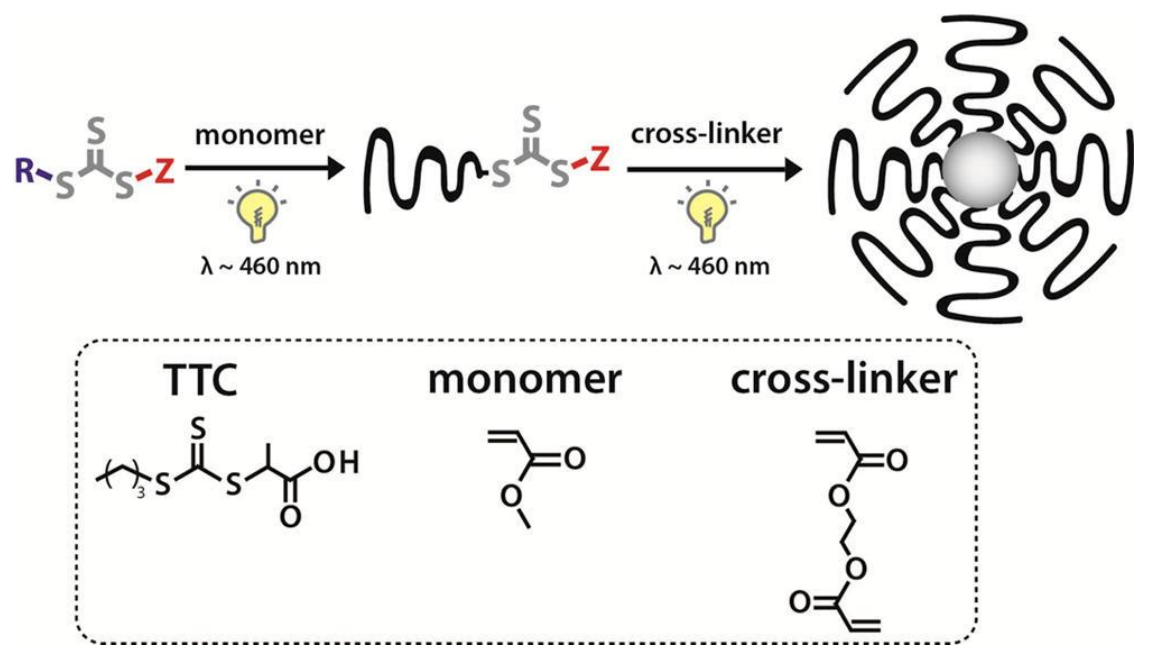

Figure 27. 


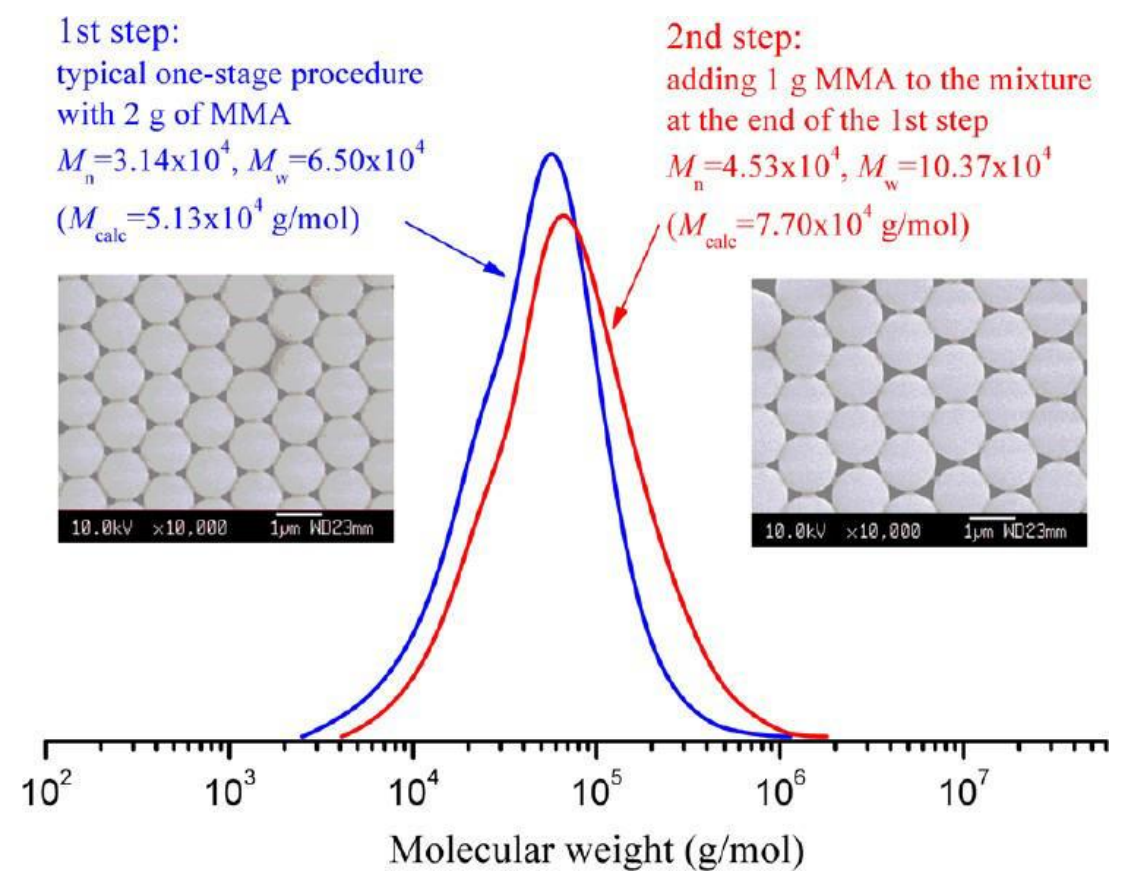

Figure 28. 
(A)

\begin{tabular}{|c|c|c|c|c|c|c|c|c|}
\hline Exp. & $\begin{array}{c}\text { Catalyst } \\
(\mathbf{p p m})\end{array}$ & $\begin{array}{c}\text { Conversion } \\
(\mathbf{\%})\end{array}$ & $\begin{array}{c}\mathbf{M}_{\mathbf{n}, \text { theo }} \\
(\mathbf{g} / \mathbf{m o l})\end{array}$ & $\begin{array}{c}\mathbf{M}_{\mathbf{n}, \mathbf{N M R}} \\
(\mathbf{g} / \mathbf{m o l})\end{array}$ & $\begin{array}{c}\mathbf{M}_{\mathbf{n}, \mathbf{G P C}} \\
(\mathbf{g} / \mathbf{m o l})\end{array}$ & $\mathbf{D}$ & $\begin{array}{c}\mathbf{D}_{\mathbf{h}} \text { (DLS) } \\
(\mathbf{n m})\end{array}$ & $\begin{array}{c}\text { PDI } \\
\text { (DLS) }\end{array}$ \\
\hline i & 20 & 41 & 23400 & 24100 & 16800 & 1.19 & 46 & 0.13 \\
\hline ii & 40 & 50 & 26600 & 25900 & 19600 & 1.21 & 134 & 0.20 \\
\hline iii & 100 & 61 & 30500 & 28600 & 22600 & 1.24 & 140 & 0.17 \\
\hline
\end{tabular}

(B)
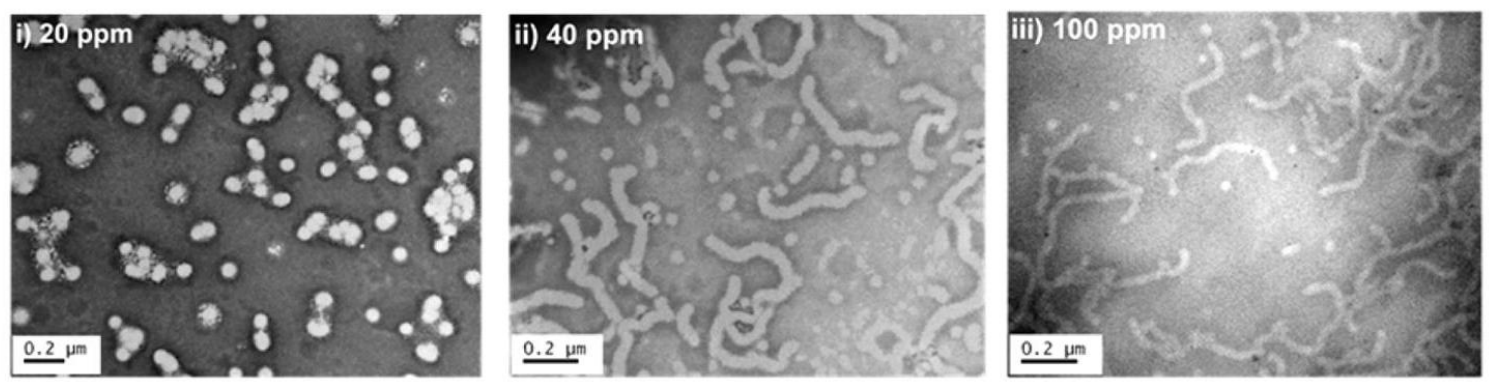

Figure 29. 


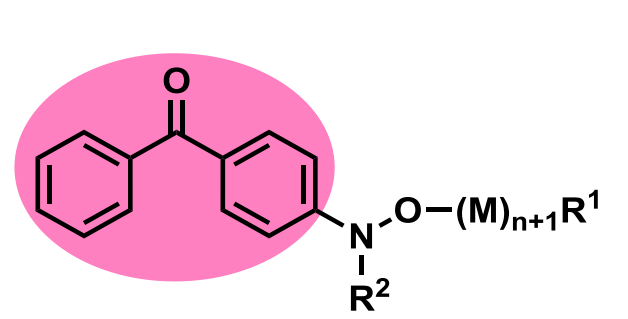

UV light

Figure 30.
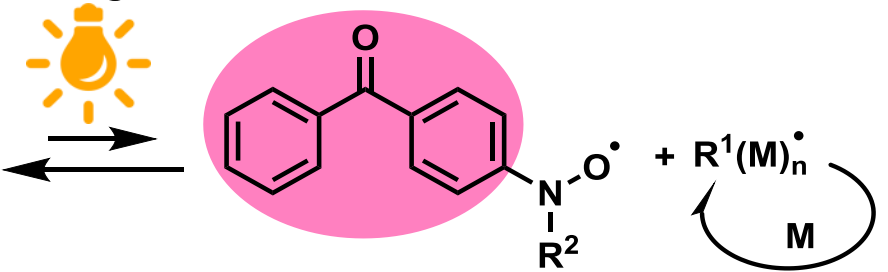


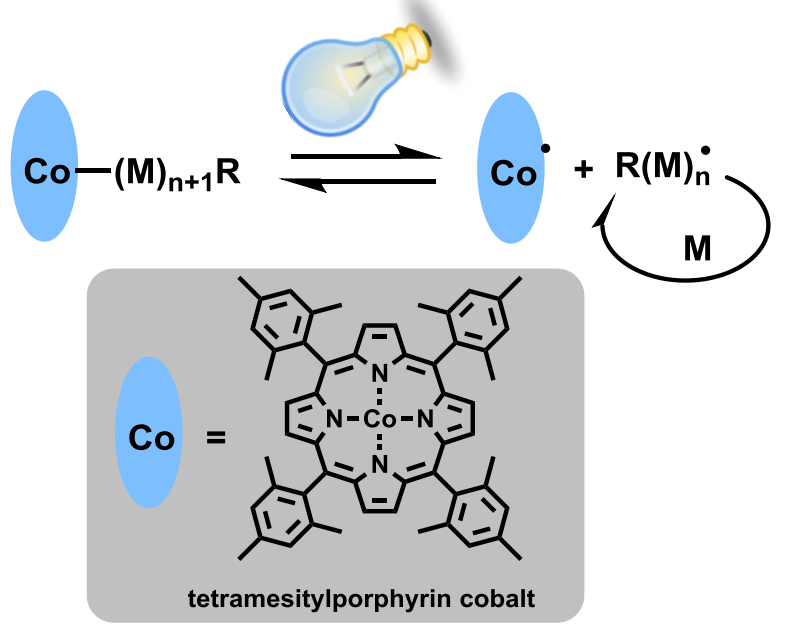

Figure 31. 


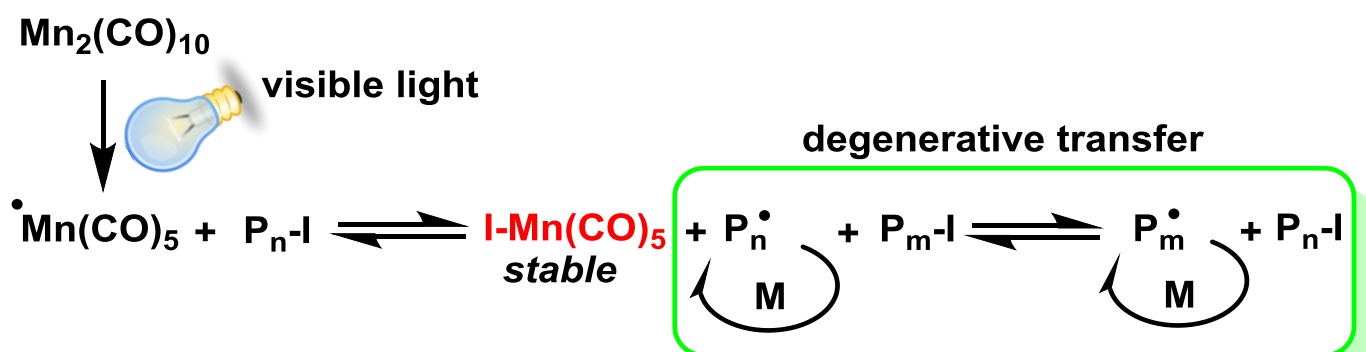

Figure 32. 


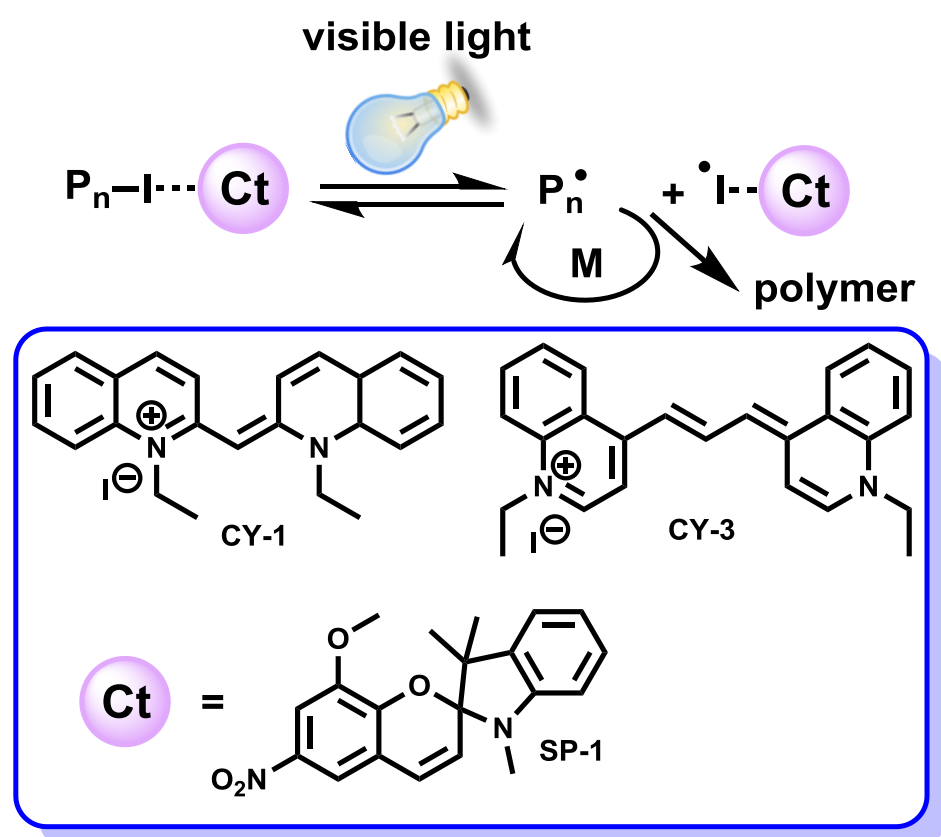

Figure 33. 


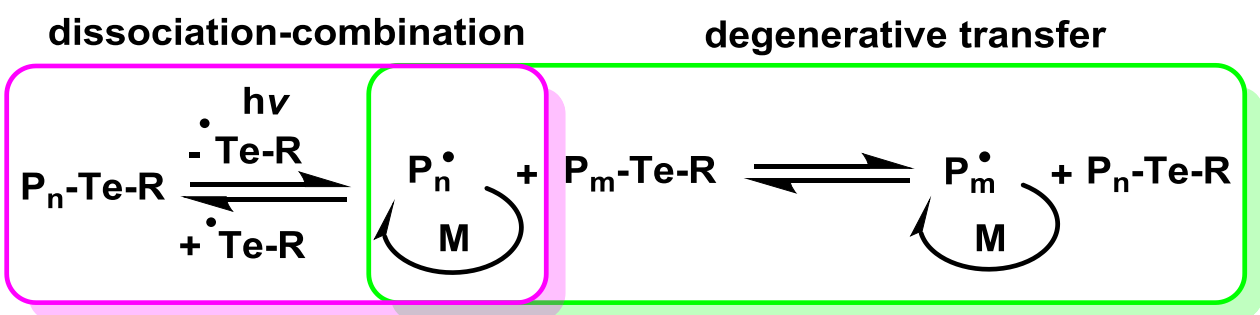

Figure 34 . 

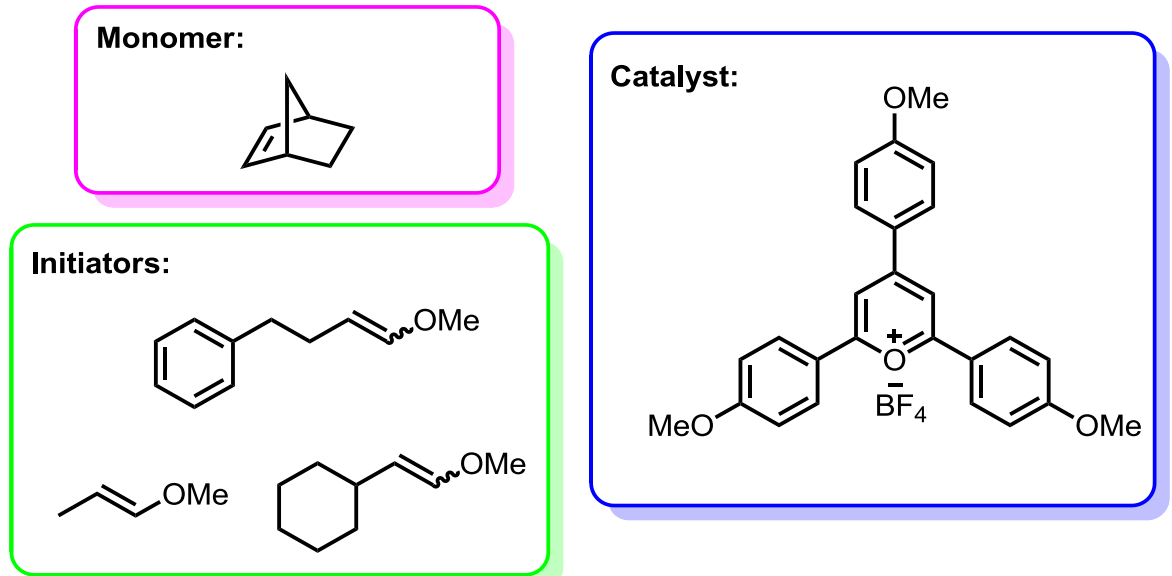

Figure 35. 


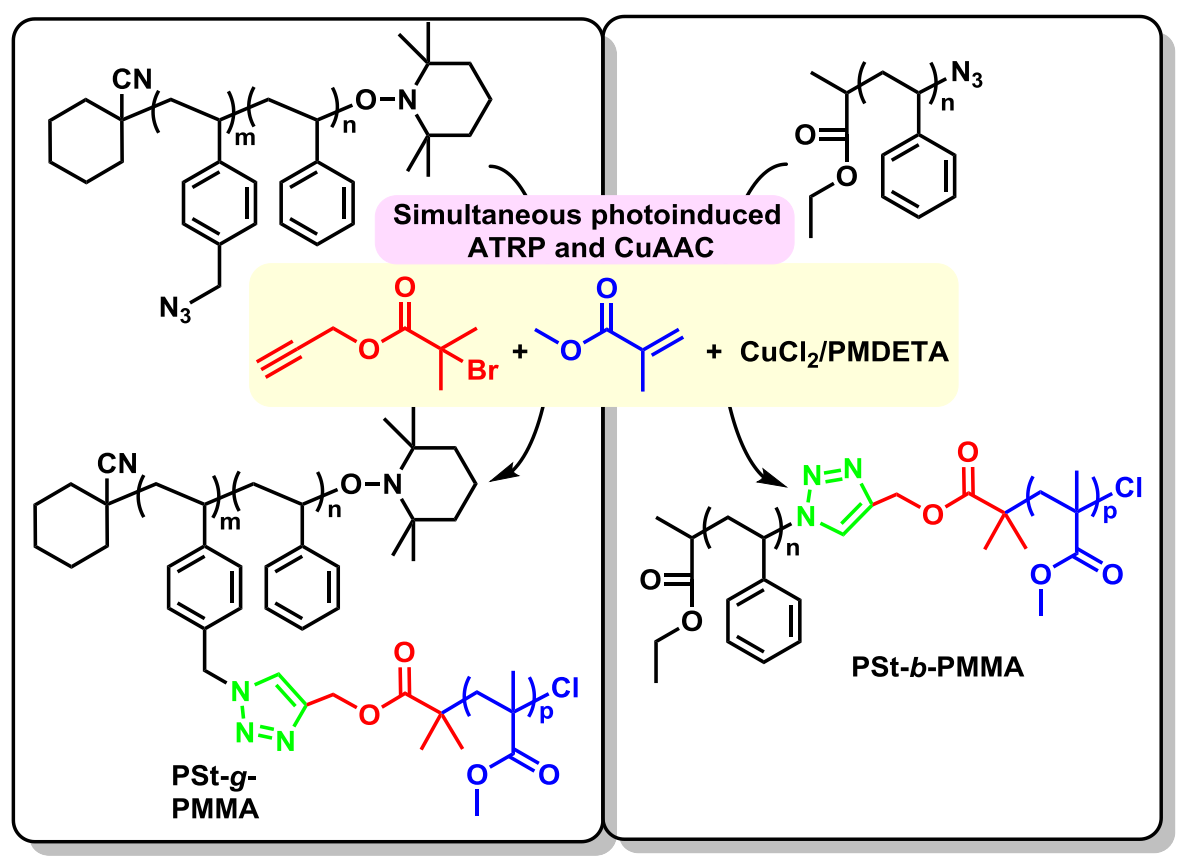

Figure 36. 

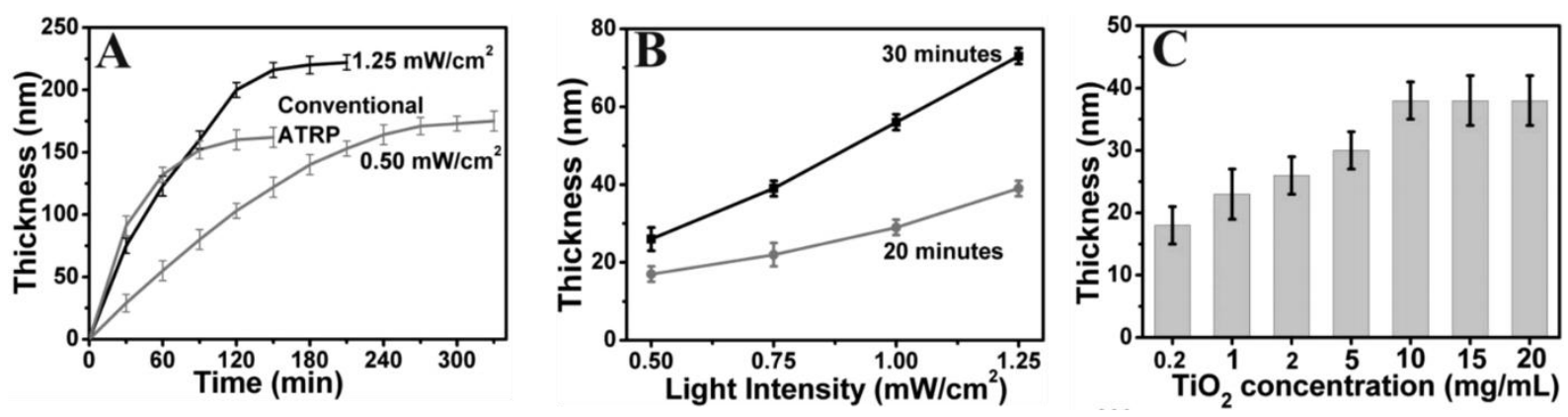

Figure 37. 

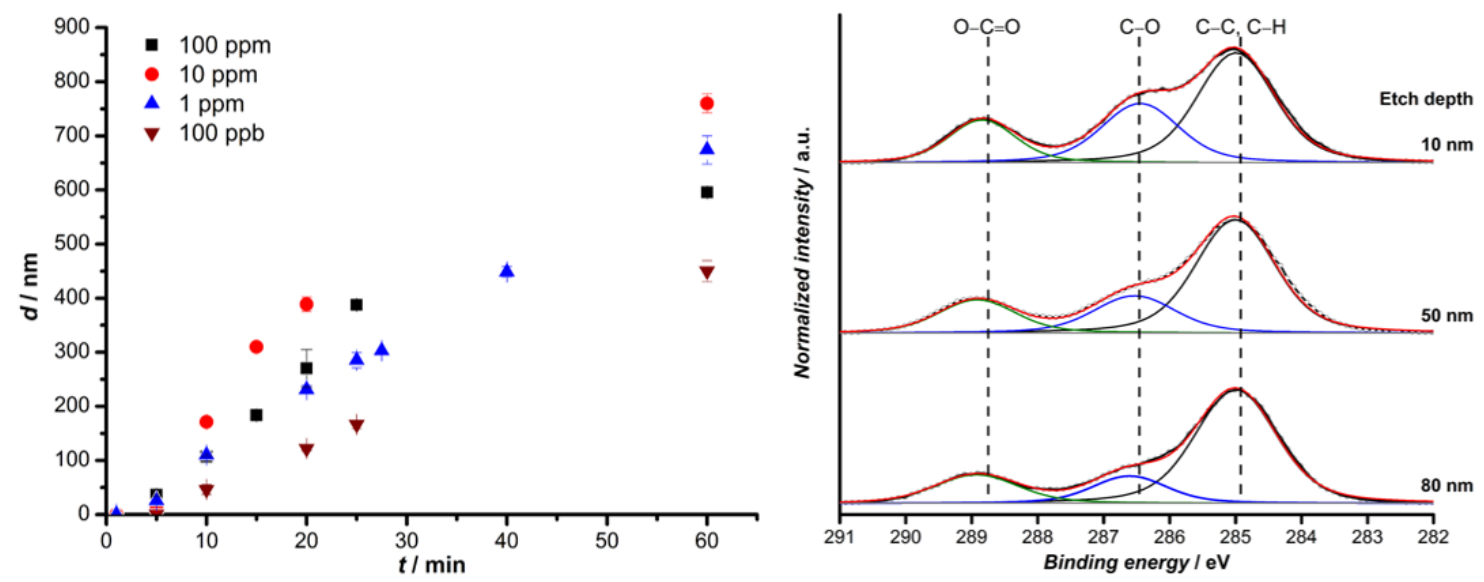

Figure 38 . 

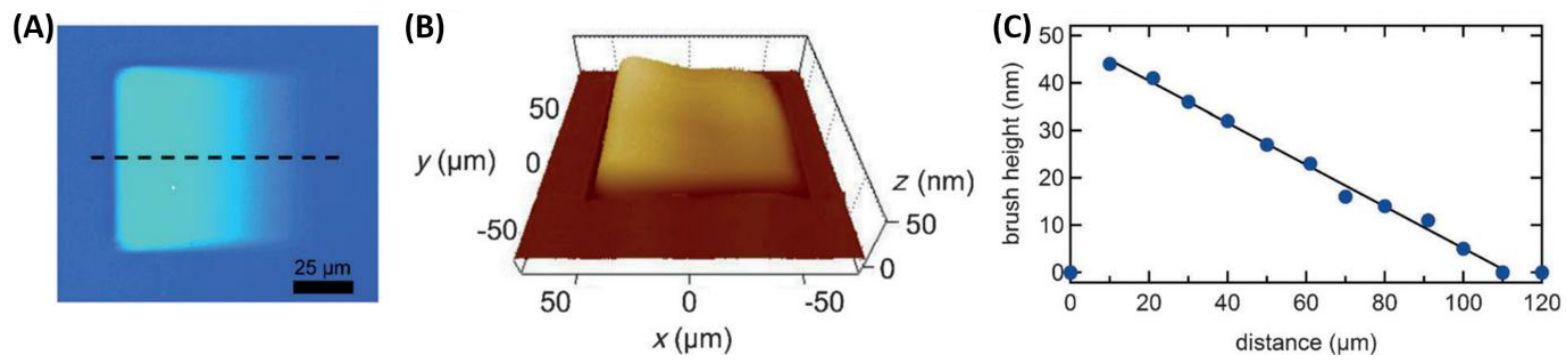

Figure 39. 

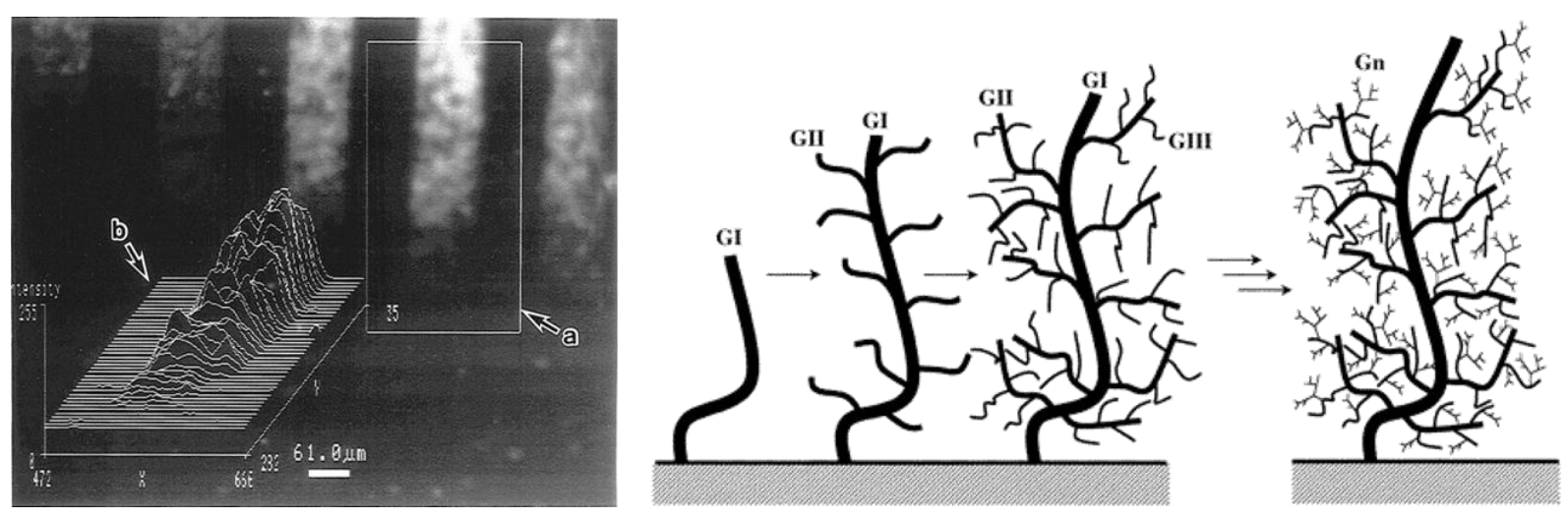

Figure 40. 


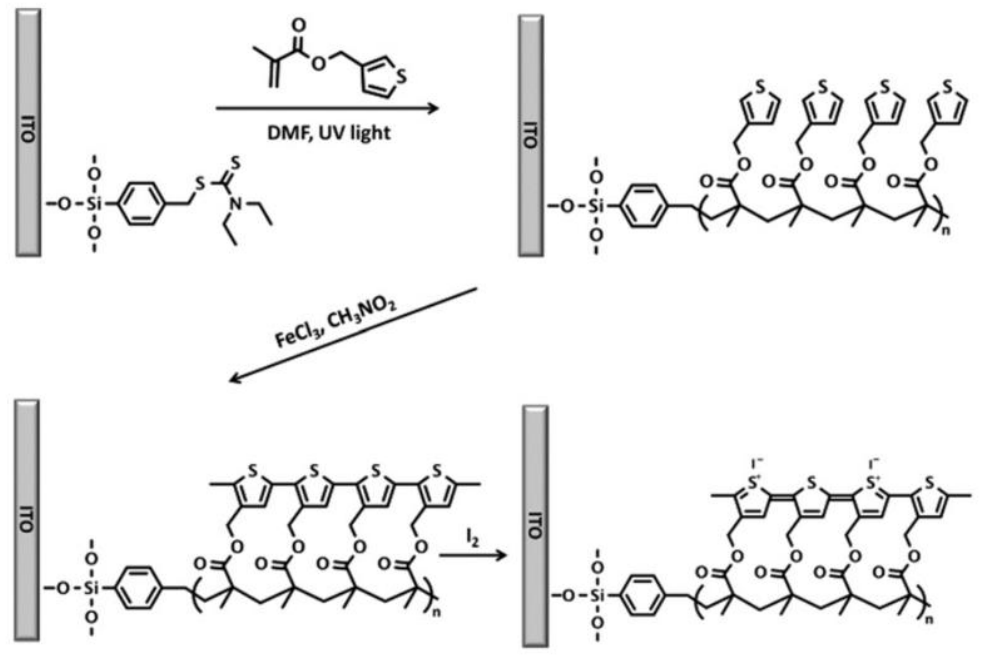

Figure 41. 

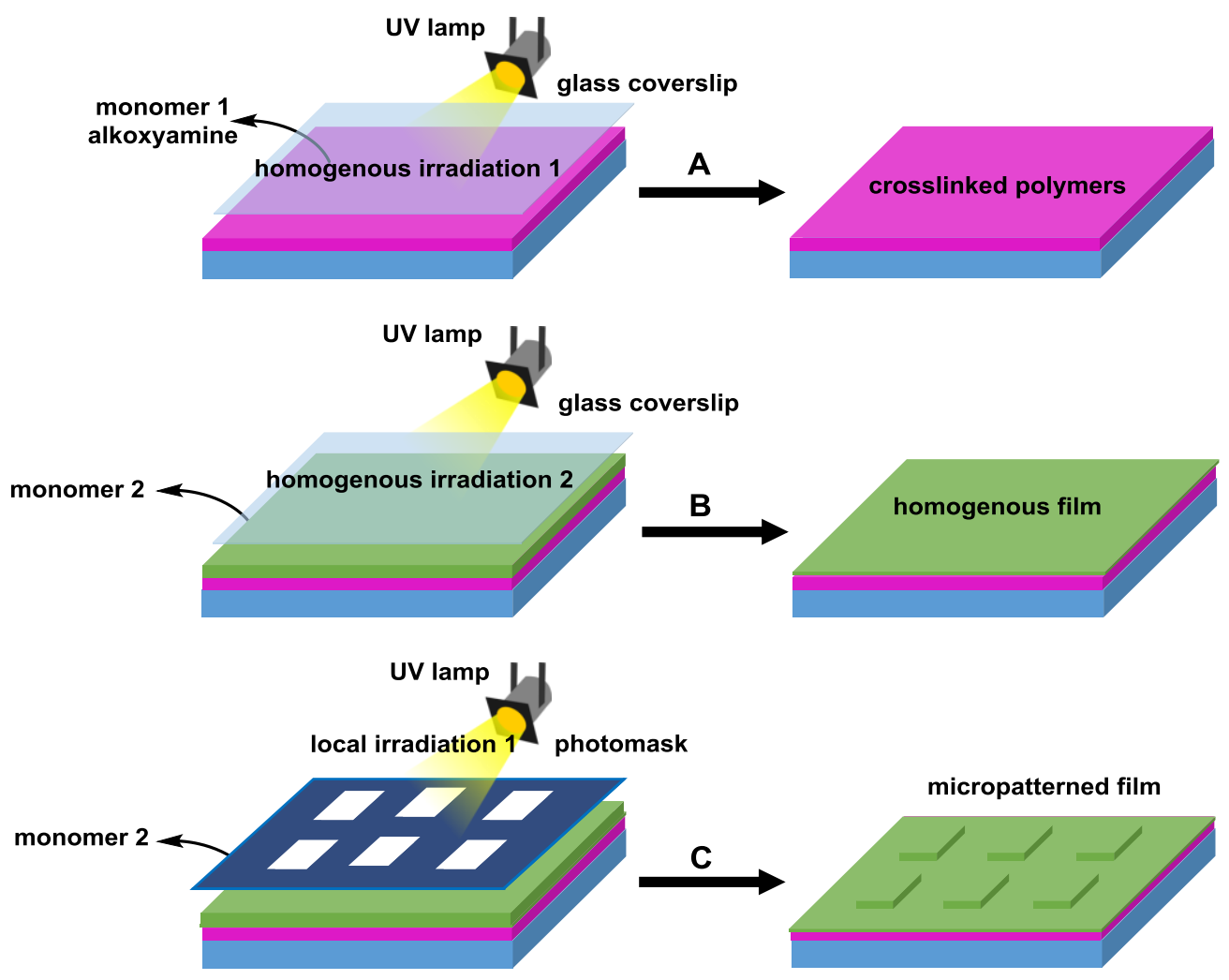

Figure 42. 

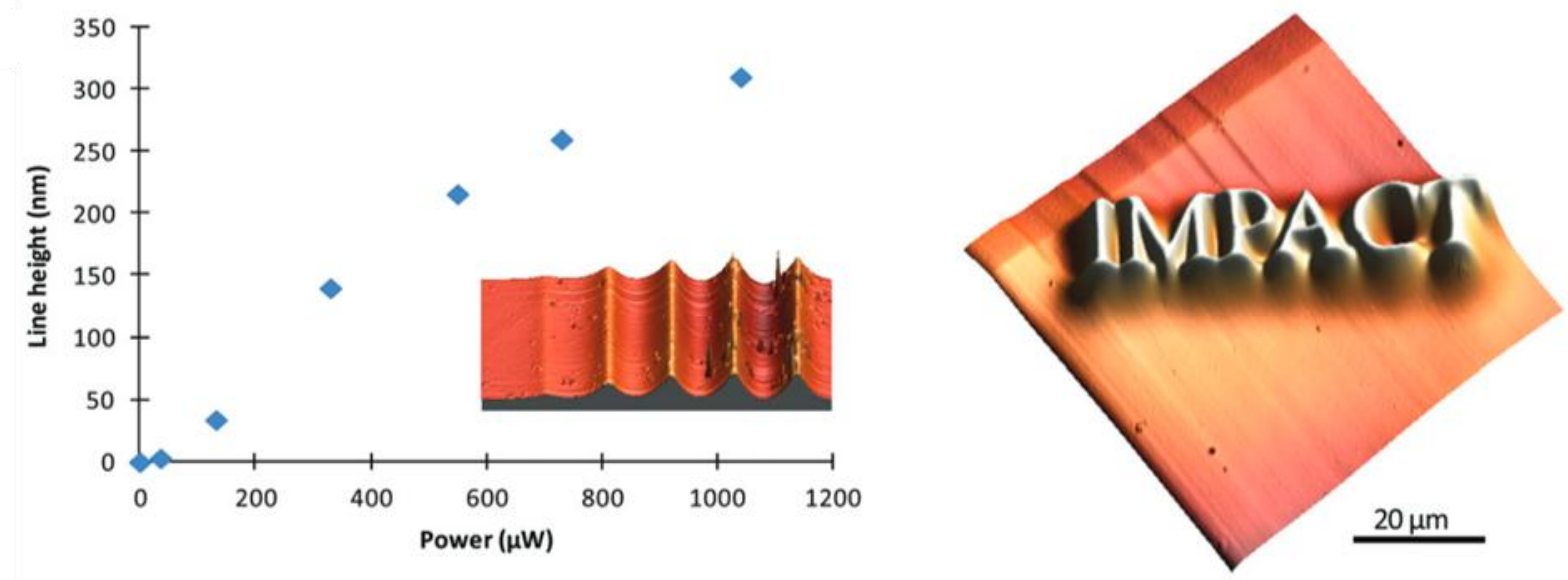

Figure 43. 
Table 1. Structure of some commercially available free radical photoinitiators.

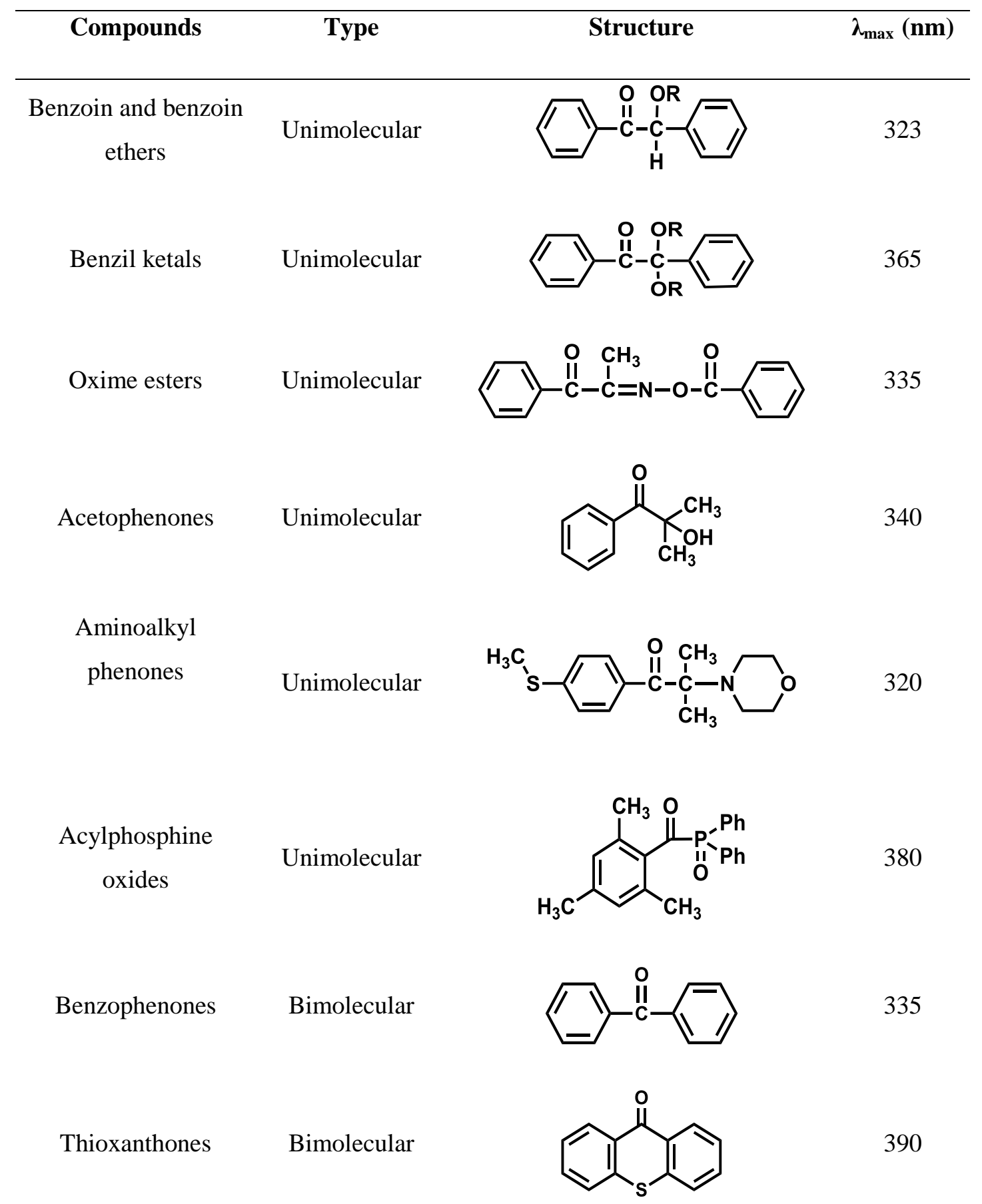


Coumarins Bimolecular

Benzils Bimolecular

Camphorquinones
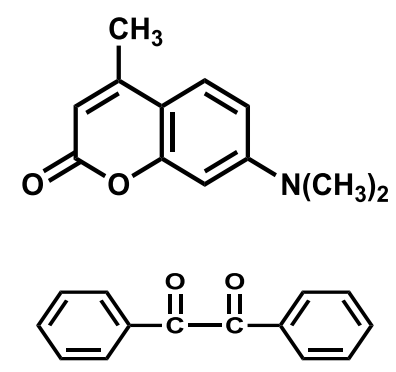

470

370

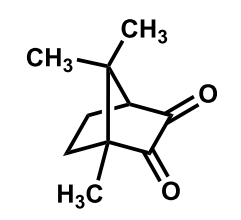


Table 2. UV and visible light sensitive compounds used in photoinduced ATRP.

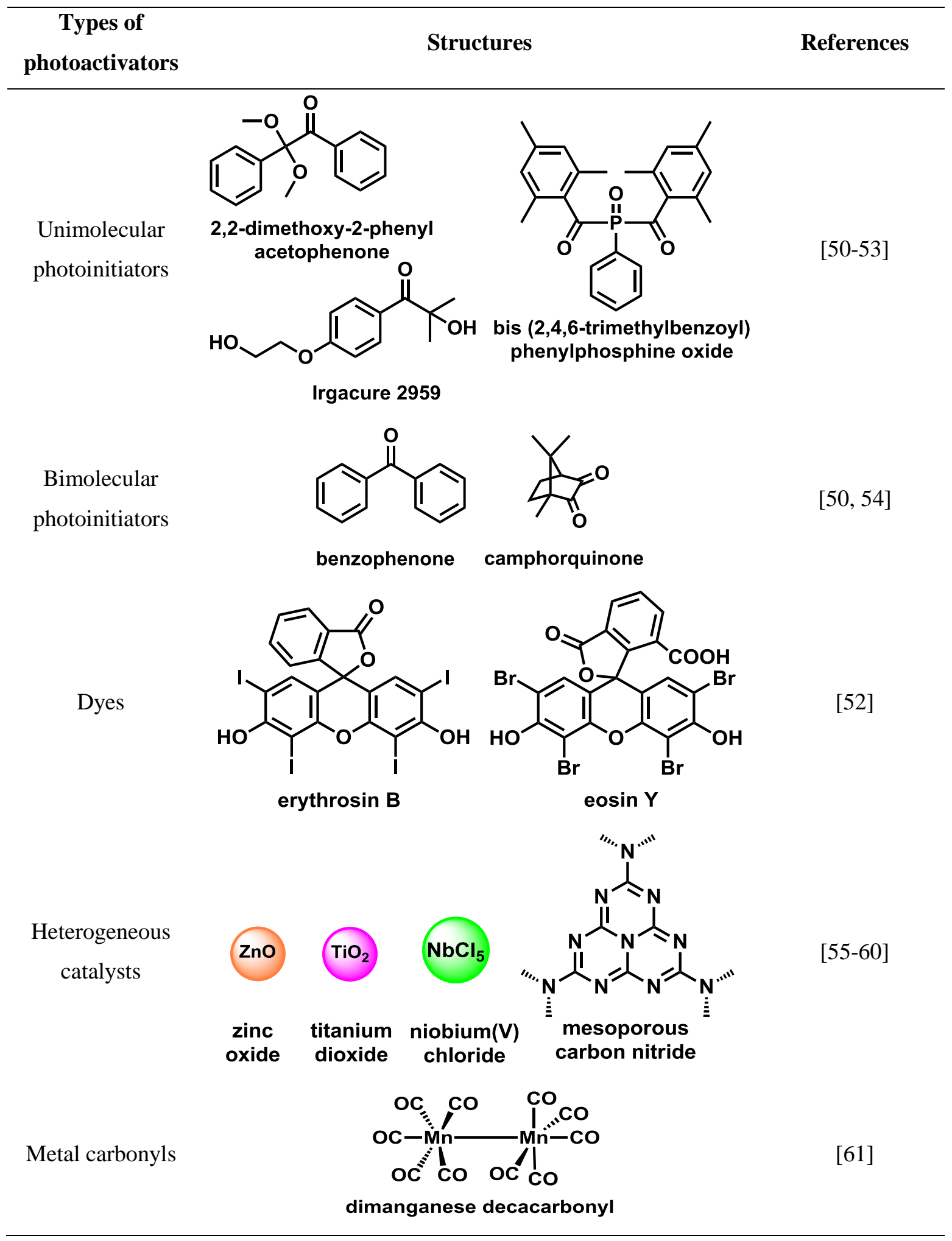


Table 3. Examples for iron-mediated photoATRP of various methacrylates.

\begin{tabular}{lllllll}
\hline Polymer & Initiator & Catalyst system & Irradiation & $M_{\mathrm{n}}$ & $M_{\mathrm{w}} / M_{\mathrm{n}}$ & {$[\mathrm{ref}]$} \\
\hline PMMA & $\mathrm{CCl}_{4}$ & $\mathrm{FeCl}_{3}, \mathrm{TMEDA}, \mathrm{AIBN}$ & $\mathrm{Hg}$ lamp $(500 \mathrm{~W})$ & 17200 & 1.26 & {$[95]$} \\
PMMA & $\mathrm{CCl}_{4}$ & $\mathrm{FeCl}_{3}, \mathrm{TMEDA}, \mathrm{BPO}$ & $\mathrm{Hg}$ lamp $(500 \mathrm{~W})$ & 15800 & 1.25 & {$[95]$} \\
PMMA & $\mathrm{CCl}_{4}$ & $\mathrm{FeCl}_{3}, \mathrm{bpy}$, ethanol & $\mathrm{Hg}$ lamp $(500 \mathrm{~W})$ & 4200 & 1.22 & {$[96]$} \\
PMMA & $\mathrm{EBiB}$ & $\mathrm{FeCl}_{3}, \mathrm{PPh}_{3}, \mathrm{Fe}_{2} \mathrm{O}_{3}$ & $\mathrm{Hg}$ lamp $(500 \mathrm{~W})$ & 11100 & 1.39 & {$[97]$} \\
PMMA & $\mathrm{EBiB}$ & $\mathrm{Fe}(\mathrm{bpy})_{3}\left(\mathrm{PF}_{6}\right)_{2}$ & $\mathrm{LED}\left(20 \mathrm{~W}, 75 \mathrm{~mW} / \mathrm{cm}^{2}\right)$ & 20200 & 1.27 & {$[98]$} \\
PGMA & $\mathrm{EBiB}$ & $\mathrm{Fe}(\mathrm{bpy})_{3}\left(\mathrm{PF}_{6}\right)_{2}$ & $\mathrm{LED}\left(20 \mathrm{~W}, 75 \mathrm{~mW} / \mathrm{cm}^{2}\right)$ & 22400 & 1.33 & {$[98]$} \\
PMMA & EBPA & $\mathrm{Fe} \mathrm{complex,} \mathrm{EDB}$ & $\mathrm{LED}(405 \mathrm{~nm})$ & 41000 & 1.80 & {$[99]$} \\
PMMA & EBPA & $\mathrm{FeBr}_{3}$ & $365 \mathrm{~nm}, 4.9 \mathrm{~mW} / \mathrm{cm}^{2}$ & 4300 & 1.18 & {$[100]$} \\
PBuMA & EBPA & $\mathrm{FeBr}_{3}$ & $365 \mathrm{~nm}, 4.9 \mathrm{~mW} / \mathrm{cm}^{2}$ & 5800 & 1.19 & {$[100]$} \\
PBnMA & EBPA & $\mathrm{FeBr}_{3}$ & $365 \mathrm{~nm}, 4.9 \mathrm{~mW} / \mathrm{cm}^{2}$ & 12700 & 1.21 & {$[100]$} \\
PMMA- $b-$ & PMMA-Br & $\mathrm{FeBr}_{3}$ & $365 \mathrm{~nm}, 4.9 \mathrm{~mW} / \mathrm{cm}^{2}$ & 9690 & 1.11 & {$[100]$} \\
PEMA & & & & & & \\
\hline
\end{tabular}


Table 4. Examples for Ir-mediated photoATRP of various methacrylates

\begin{tabular}{lllllll}
\hline Polymer & Initiator & Catalyst system & Irradiation & $M_{\mathrm{n}}$ & $M_{\mathrm{w}} / M_{\mathrm{n}}$ & [ref] \\
\hline PMMA & EBPA & $f a c-\left[\operatorname{Ir}(\mathrm{ppy})_{3}\right]$ & $435 \mathrm{~nm} \operatorname{LED}\left(0.5 \mathrm{~mW} / \mathrm{cm}^{2}\right)$ & 22900 & 1.25 & {$[119]$} \\
PMAA & EBPA & $f a c-\left[\operatorname{Ir}(\mathrm{ppy})_{3}\right]$ & $435 \mathrm{~nm} \operatorname{LED}\left(0.5 \mathrm{~mW} / \mathrm{cm}^{2}\right)$ & 28000 & 1.61 & {$[119]$} \\
PEGMA & EBiB & $f a c-\left[\operatorname{Ir}(\mathrm{ppy})_{3}\right]$ & $420 \mathrm{~nm} \operatorname{LED}\left(4.5 \mathrm{~mW} / \mathrm{cm}^{2}\right)$ & $6000-$ & $1.11-$ & {$[121]$} \\
& & & 19000 & 1.26 & \\
PTFEMA & $\mathrm{C}_{6} \mathrm{~F}_{13} \mathrm{I}$ & $f a c-\left[\operatorname{Ir}(\mathrm{ppy})_{3}\right]$ & $420 \mathrm{~nm} \operatorname{LED}\left(3.2 \mathrm{~mW} / \mathrm{cm}^{2}\right)$ & 22000 & 1.23 & {$[122]$} \\
PPMA-b- & EBPA & $f a c-\left[\operatorname{Ir}(\mathrm{ppy})_{3}\right]$ & $420 \mathrm{~nm} \operatorname{LED}\left(4.5 \mathrm{~mW} / \mathrm{cm}^{2}\right)$ & 8300 & 1.24 & {$[123]$} \\
PMMA & & & & & & \\
PMA & $\mathrm{BnBiB}$ & $f a c-\left[\operatorname{Ir}(\mathrm{ppy})_{3}\right]$ & $380 \mathrm{~nm} \operatorname{LED}\left(0.65 \mu \mathrm{W} / \mathrm{cm}^{2}\right)$ & 8200 & 1.19 & {$[124]$} \\
PEA-b-PAA & $\mathrm{BnBiB}$ & $f a c-\left[\operatorname{Ir}(\mathrm{ppy})_{3}\right]$ & $380 \mathrm{~nm} \operatorname{LED}\left(0.65 \mu \mathrm{W} / \mathrm{cm}^{2}\right)$ & 16300 & 1.65 & {$[124]$} \\
PMMA & $\mathrm{EBPA}$ & $\operatorname{Ir}(\mathrm{btp})_{2}(\mathrm{tmd})$ & $462 \mathrm{~nm} \operatorname{LED}\left(10 \mathrm{~mW} / \mathrm{cm}^{2}\right)$ & 25140 & 1.2 & {$[126]$} \\
\hline
\end{tabular}


Table 5. Examples for PI-initiated photoRAFT polymerizations of various monomers.

\begin{tabular}{cccccc}
\hline Polymer & PI & RAFT agent & $M_{\mathrm{n}}$ & $\oplus$ & [ref] \\
\hline PS & TPO & BDMAT & 2700 & 1.26 & {$[191]$} \\
PS-b-PVBA & TPO & BDMAT & 15500 & 1.15 & {$[191]$} \\
PNIPAAm & Irgacure 2959 & BDMAT & 13500 & 1.17 & {$[195]$} \\
PGMA & TPO & CPFDB & 36100 & 1.18 & {$[193]$} \\
PBMA & TPO & CPFDB & 6800 & 1.20 & {$[193]$} \\
PBMA-b-PGMA & TPO & CPFDB & 27800 & 1.17 & {$[193]$} \\
PBA & TPO & BDMAT & 80000 & 1.3 & {$[192]$} \\
PBA-b-PMA-b-PBA & TPO & BDMAT & $-^{\mathrm{a}}$ & $-{ }^{\mathrm{a}}$ & {$[192]$} \\
PNAP & TPO & EDMAT & 6450 & 1.10 & {$[194]$} \\
PNAP-b-PPEGA & TPO & EDMAT & - -- $^{\mathrm{a}}$ & $-{ }^{\mathrm{a}}$ & {$[194]$} \\
& BAPO & MESA & 10500 & 1.23 & {$[196]$}
\end{tabular}

${ }^{\mathrm{a}}$ Good control over the block extension is seen, however, no $M_{\mathrm{n}}$ data was given in the original publication 
Table 6. Examples for initiator-free photoRAFT polymerizations of various monomers.

\begin{tabular}{ccccc}
\hline Polymer & RAFT agent & $M_{\mathrm{n}}$ & $\oplus$ & [ref] \\
\hline PAA & BDMAT & 39000 & 1.08 & {$[201]$} \\
PBA & BDMAT & 37700 & 1.05 & {$[203]$} \\
PS & BDMAT & 15600 & 1.12 & {$[203]$} \\
PS- $b$-PBA- $b$-PS & BDMAT & 210000 & 1.15 & {$[203]$} \\
PVAc & CPEC & 16900 & 1.38 & {$[205]$} \\
PVAc & MESA & 10600 & 1.24 & {$[205]$} \\
PMA & DDMAT & 53700 & 1.19 & {$[206]$} \\
PMA & DDMAT & 9300 & 1.06 & {$[208]$} \\
PNIPAAm & DDMAT & 55800 & 1.12 & {$[208]$} \\
PMA & DDMAT & $\sim 80000$ & $<1.15$ & {$[208]$}
\end{tabular}

${ }^{a}$ Polymer was obtained by adding fresh monomer five times at $>90 \%$ monomer conversion. The polymer can hence be defined as a pseudohexablock copolymer. 
Table 7. Examples for PET-RAFT polymerizations of various monomers.

\begin{tabular}{|c|c|c|c|c|c|}
\hline Polymer & PET catalyst & RAFT agent & $M_{\mathrm{n}}$ & 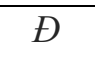 & [ref] \\
\hline PMA & $f a c-\left[\operatorname{Ir}(\mathrm{ppy})_{3}\right]$ & BTPA & 17500 & 1.05 & [214] \\
\hline PMMA & $f a c-\left[\operatorname{Ir}(\mathrm{ppy})_{3}\right]$ & CPADB & 335000 & 1.23 & [214] \\
\hline РMМА- $b$-РНРМА & $f a c-\left[\operatorname{Ir}(\mathrm{ppy})_{3}\right]$ & BTPA & 31330 & 1.16 & [214] \\
\hline PDMA & $\mathrm{Ru}(\mathrm{bpy})_{3}$ & BTPA & 15900 & 1.09 & [218] \\
\hline PMA & $\mathrm{Ru}(\mathrm{bpy})_{3}$ & BTPA & 28900 & 1.11 & [218] \\
\hline PMMA & Eosin $Y$ & CPADB & 14900 & 1.21 & [220] \\
\hline PMMA & Chlorophyll & CPADB & 20400 & 1.16 & [222] \\
\hline PHEMA & Chlorophyll & CPADB & 22700 & 1.08 & [222] \\
\hline PMA & porphyrin & BTPA & 10500 & 1.07 & [221] \\
\hline PNIPAAM & curcumin & Not given & 43500 & 1.12 & [224] \\
\hline PNIPAAM & PTZ & Symmetrical TTC & 23200 & 1.02 & [227] \\
\hline PNIPAAM & $\mathrm{NbBA}$ & CETP & 45500 & 1.18 & [226] \\
\hline PAA & NbBA & CETP & 16800 & 1.20 & [226] \\
\hline PPEGA & $\mathrm{NbBA}$ & CETP & 89000 & 1.22 & [226] \\
\hline PMMA & NbBA & CETP & 18200 & 1.16 & [226] \\
\hline PNVC & $\mathrm{Mn}_{2}(\mathrm{CO})_{10}$ & CPDN & 2600 & 1.22 & [225] \\
\hline PMA & $\mathrm{Mn}_{2}(\mathrm{CO})_{10}$ & CPDN & 15900 & 1.10 & [225] \\
\hline PDMAEMA & $\mathrm{Mn}_{2}(\mathrm{CO})_{10}$ & CPDN & 19600 & 1.37 & [225] \\
\hline
\end{tabular}


Table 8. Examples of photosensitive alkoxyamines used in the NMP process.

\begin{tabular}{ccc}
\hline Structures & Conditions & Results and References \\
\hline Methyl methacrylate, & $\begin{array}{c}\text { Molecular weight increase } \\
\text { UV light }\end{array}$ & $\begin{array}{c}\text { with monomer } \\
\text { concentration }\end{array}$ \\
(Rayonet reactor) & $12 \mathrm{~h}$ & [253]
\end{tabular}<smiles>Cc1nc2ccccc2c(C(=O)OC2CC(C)(C)N(OC(C)c3ccccc3)C(C)(C)C2)c1O</smiles>

Styrene,

$$
\begin{aligned}
& \text { UV light (300-400 nm) } \quad 10 \% \text { conversion, } \\
& 175 \mathrm{~W} \text { xenon lamp with } \quad M_{\mathrm{n}}=2700 \\
& \text { cutoff filters and } M_{\mathrm{w}} / M_{\mathrm{n}}=1.60[252] \\
& 2 \mathrm{~h} \\
& \text { n-Butyl acrylate } \quad 30 \% \text { conversion, } \\
& \text { UV light } \quad M_{\mathrm{n}}=250,000 \\
& \left(22 \mathrm{~mW} \mathrm{~cm}^{-2}\right) \quad \text { and } M_{\mathrm{w}} / M_{\mathrm{n}}=2.50 \text { [172] } \\
& 500 \mathrm{~s} \\
& \text { n-Butyl acrylate } \quad 30-80 \% \text { conversion, } \\
& \text { UV light } \quad M_{\mathrm{n}}=25,000-40,000 \\
& \left(22 \mathrm{~mW} \mathrm{~cm}^{-2}\right) \quad \text { and } M_{\mathrm{w}} / M_{\mathrm{n}}=2.20-3.90 \\
& 500 \mathrm{~s} \quad \text { [172] } \\
& \text { n-Butyl acrylate, UV light } \\
& \left(44 \mathrm{~mW} \mathrm{~cm}^{-2}\right) \\
& 1000 \mathrm{~s} \\
& \text { Isobornyl acrylate } \\
& \text { UV light } \\
& 5 \mathrm{~min} \\
& M_{\mathrm{n}}=25,000-40,000
\end{aligned}
$$<smiles>CON1C(C)(C)CC(OC(=O)c2cccc(C(=O)c3ccccc3)c2)CC1(C)C</smiles> 
$45 \mathrm{~min}$

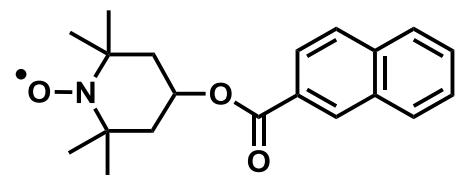

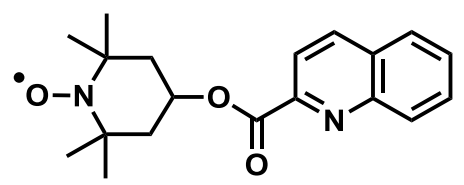

DMPA, MMA,

UV light

$\left(3 \mathrm{~mW} \mathrm{~cm}^{-2}\right.$ )

$45 \mathrm{~min}$

DMPA, MMA,

UV light

$\left(3 \mathrm{~mW} \mathrm{~cm}^{-2}\right)$

$45 \min$
[58]

14-79\% conversion

$$
M_{\mathrm{n}}=1,800-10,000
$$

and $M_{\mathrm{w}} / M_{\mathrm{n}}=1.34-2.39$

[58]

$15-58 \%$ conversion

$M_{\mathrm{n}}=2,000-7,500$

and $M_{\mathrm{w}} / M_{\mathrm{n}}=1.33-1.37$

[58] 